\title{
On the action of the group of automorphisms of the affine plane on instantons
}

\author{
Dissertation \\ zur Erlangung des mathematisch-naturwissenschaftlichen Doktorgrades \\ "Doctor rerum naturalium" \\ der Georg-August-Universität Göttingen
}

vorgelegt von

Michael Miesener

aus Rinteln

Göttingen 2010 
Refenrent: Prof. Dr. Victor Pidstsrygach

Koreferent: Prof. Dr. Hans Christian Graf v. Bothmer Tag der mündlichen Prüfung: 21. Dezember 2010 


\section{Contents}

1 Introduction $\quad 5$

2 Tools and notations $\quad 8$

2.1 support, setsupport and Fitting scheme . . . . . . . . . . . 8

2.2 Torsion sheaves . . . . . . . . . . . . . . . . . . . . . . 9 9

2.3 Duality, Ext and Ext . . . . . . . . . . . . . . . . . . 10

2.4 Torsion and duality for sheaves of rank 0 . . . . . . . . . . . . 11

2.5 Further tools . . . . . . . . . . . . . . . . . . . . . 11

2.6 Grothendieck and Chern classes . . . . . . . . . . . . . . 12

2.7 The Grothendieck spectral sequence . . . . . . . . . . . . 15

3 The action of the group of biregular automorphism of the affine $\begin{array}{ll}\text { plane on instantons } & 16\end{array}$

4 Instantons as vector bundles on $\mathbb{P}_{2} \quad 22$

5 The $\mathfrak{G}$-action on $\mathcal{M}_{c}^{\text {reg }}(n)$ for $n \leq 4 \quad 29$

$5.1 \tilde{\mathcal{M}}_{c}^{r e g}(n)$ and $\tilde{\mathcal{M}}_{n c}^{r e g}(n) \ldots \ldots \ldots \ldots . \ldots \ldots 29$

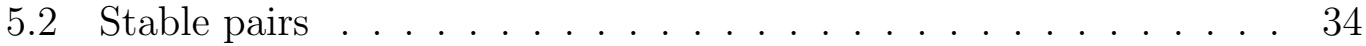

$5.3 \tilde{\mathcal{M}}_{c}^{r e g}(2)$ and $\tilde{\mathcal{M}}_{c}^{r e g}(3) \ldots \ldots \ldots \ldots . \ldots \ldots$

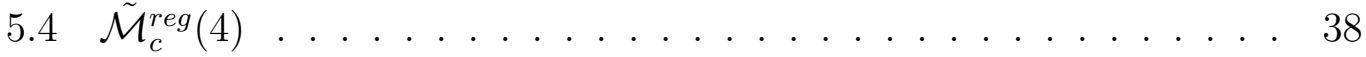

6 The $\mathfrak{G}$-action on $\mathcal{M}_{n c}^{r e g}(2)$ and $\mathcal{M}_{n c}^{r e g}(3) \quad 43$

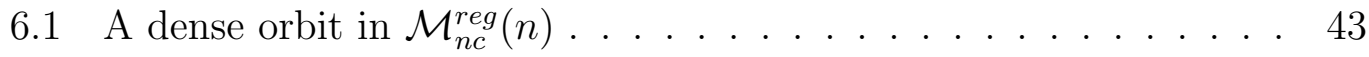

6.2 The spectral scheme . . . . . . . . . . . . . . . . . . . . 44

6.3 Spectral schemes of elements of $\tilde{\mathcal{M}}_{n c}^{r e g}(2)$ and $\tilde{\mathcal{M}}_{n c}^{r e g}(3) \ldots . . . \quad 47$

7 Stability of locally free sheaves corresponding to instantons $\quad 52$

7.1 Stability of torsion free sheaves . . . . . . . . . . . . . . . . 52

7.1.1 Stability on curves . . . . . . . . . . . . . . . . 52

7.1.2 Stability on $\mathbb{P}_{m} \ldots \ldots . \ldots . \ldots . . \ldots 53$ 
$7.2 \mathfrak{G}$ and the Cremora group . . . . . . . . . . . . . 56

$8 \tilde{\mathcal{M}}_{n c}^{r e g}(4) \quad \mathbf{5 9}$

8.1 Coherent systems . . . . . . . . . . . . . . . . . . 59

8.2 Instantons and coherent systems . . . . . . . . . . . . 61

8.3 Spectral schemes and coherent systems . . . . . . . . . . . . 69

8.4 Detecting the spectral scheme . . . . . . . . . . . . . . 75

8.4.1 Nonsingular conics . . . . . . . . . . . . . . . . 78

8.4 .2 Singular conics . . . . . . . . . . . . . . 80

8.4 .3 Lines ...................... 87

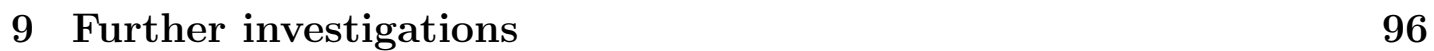




\section{Chapter 1}

\section{Introduction}

We consider the set of $S U(2)$-instantons on $\mathbb{R}^{4}$ with finite energy, which are antiself-dual connections of $S U(2)$-bundles over $\mathbb{R}^{4}$ (see [DK]). Since such a connection can be extended to a connection on $S^{4}$ due to the removeable singularities theorem of Uhlenbeck, the famous ADHM-construction [DK] shows that there is a 1-to-1 correspondence between $S U(2)$-instantons of charge $n$ with framing at the fibre at infinity (the fibre resulting from compactifying $\mathbb{R}^{4}$ to $S^{4}$ ) and

$$
\mathcal{M}^{\text {reg }}(n)=\left\{\begin{array}{ll} 
& (i)[A, B]+i j=0 \\
(A, B, i, j) \mid & \text { (ii) }\left[A, A^{*}\right]+\left[B, B^{*}\right]+i i^{*}-j^{*} j=0 \\
\text { (iii) } \operatorname{Stab}_{U(n)}(A, B, i, j)=\mathbf{1}
\end{array}\right\} / U(n)
$$

where $A, B \in \operatorname{End}(V), i \in \operatorname{Hom}(W, V)$ and $j \in \operatorname{Hom}(V, W)$ with $V$ and $W$ hermitian vector spaces of dimension $n$ and 2 respectively and $U(n)$-action, which is given by the $G L(n)$-action

$$
g(A, B, i, j)=\left(g^{-1} A g, g^{-1} B g, g^{-1} i, j g\right) \text { for } g \in G L(n) .
$$

One can show that these $U(n)$-orbits of ADHM data are in 1-to-1 correspondence to $G L(n)$-orbits of tuples $(A, B, i, j)$ satisfying

$$
[A, B]+i j=0
$$

and the two stability conditions 
(S1) \# proper subspace $S \subset V$ such that $A(S) \subset S ; B(S) \subset S$ and $i m(i) \subset S$

(S2) $\nexists$ proper subspace $S \subset V$ such that $A(S) \subset S ; B(S) \subset S$ and $S \subset \operatorname{ker}(j)$

(see $\S 3$ ). An action of $\mathfrak{G}$, the group of regular automorphism of $\mathbb{C}^{2}$, arises in two natural ways: through the action on the above matrix data and through the action on locally free sheaves on $\mathbb{P}_{2}$. Since $\mathfrak{G}=\mathcal{A}_{2} *_{\Gamma} \mathfrak{E}$, where $\mathcal{A}_{2}$ are the affine, $\mathfrak{E}$ the elementry transformations and $\Gamma=\mathcal{A}_{2} \cap \mathfrak{E}$, the first action is given by

$$
\begin{gathered}
a(A, B, i, j)=\left(\mu_{0} A+\mu_{1} B+\mu_{2} \mathbf{1}, \nu_{0} A+\nu_{1} B+\nu_{2} \mathbf{1}, \operatorname{det}(a) i, j\right), \\
e_{p}(A, B, i, j)=(A, B+p(A), i, j),
\end{gathered}
$$

where $a \in \mathcal{A}_{2}$ and $e_{p} \in \mathfrak{E}$ with $p \in \mathbb{C}[t]$. To get the second action one has to consider an inclusion $\mathbb{A}_{2} \hookrightarrow \mathbb{P}_{2}$ which implies an inclusion of $\mathfrak{G}$ into $\operatorname{Cr}\left(\mathbb{P}_{2}\right)$, the set of biregular automorphism on $\mathbb{P}_{2}$, and identify $S U(2)$-instantons with the set of locally free sheaves $E$ on $\mathbb{P}_{2}$ trivial at $l_{\infty}:=\mathbb{P}_{2} \backslash \mathbb{A}_{2}$, satisfying $c_{1}(E)=$ $0, c_{2}(E)=n$ (see $\S 4$ ). Then for $g \in \mathfrak{G}$ one defines $g(E):=g_{*}(E)$ (more in $\S 7$ ). Considering these two actions one has

Theorem 1.0.1 The bijection between the set of locally free sheaves $E$ on $\mathbb{P}_{2}$ trivial at $l_{\infty}$, satisfying $c_{1}(E)=0, c_{2}(E)=n$ and the set of $G L(n) \times G L(2)$ orbits of ADHM data is $\mathfrak{G}$-equivariant.

(see $\S 7)$.

In this work we investigate the $\mathfrak{G}$-action for charge $n \leq 4$. The basis for this is a lemma of Artamkin [A1], which states that in the set of pairs $(A, B)$, such that their commutators are equal up to a factor, there is a dense $\mathfrak{G}$-orbit, which includes the pairs having no common invariant subspace and a reduced spectral scheme. The spectral scheme of a pair $(A, B)$ is the subscheme of $\mathbb{P}_{2}=\operatorname{Proj}\left(\mathbb{C}\left[\lambda_{0}, \lambda_{1}, \lambda_{2}\right]\right)$, which is given by the homogeneous ideal generated by $\operatorname{det}\left(\lambda_{0} A+\lambda_{1} B-\lambda_{2} \mathbf{1}\right)$.

In $\S 3$ we show that $S U(2)$-instantons can be described as stable $G L(n)$-orbits of tuples $(A, B, i, j)$ satisfying $[A, B]+i j=0$, give the $\mathfrak{G}$-action and show that it is well defined. In $\S 4$ we show that by dropping stability condition (S2) in 1.0.1 we extend the moduli space $\mathcal{M}^{\text {reg }}(n)$ to include torsion free sheaves on $\mathbb{P}_{2}$, with trivialisation on $l_{\infty}$.

In $\S 5$ we consider the space of $S U(2)$-instantons of charge $n$ modulo framing at infinity, which we denote by $\tilde{\mathcal{M}}^{\text {reg }}(n)$. We show, that there is no instanton 
with $\operatorname{ADHM}$ datum $(A, B, i, j)$ such that $r k[A, B]=1$. We therefore get a decomposition as disjoint union

$$
\tilde{\mathcal{M}}^{r e g}(n)=\tilde{\mathcal{M}}_{c}^{r e g}(n) \sqcup \tilde{\mathcal{M}}_{n c}^{r e g}(n)
$$

where $\tilde{\mathcal{M}}_{c}^{r e g}(n)$ denotes the $G L(n) \times G L(2)$-orbits of ADHM data with $[A, B]=0$ and $\tilde{\mathcal{M}}_{n c}^{r e g}(n)$ those with $[A, B] \neq 0$. Furthermore we show that an element of $\tilde{\mathcal{M}}_{n c}^{\text {reg }}(n)$ is uniquely determined by a pair $(A, B)$, and that up to a factor there are $n-1$ conjugation classes of the commutator $[A, B]$ for $n \leq 4$ and 3 conjugation classes for $n>4$. Therefore Artamkins theorem implies that there are at least $n-1 \mathfrak{G}$-orbits in $\tilde{\mathcal{M}}_{n c}^{r e g}(n)$ for $n \leq 4$. Before answering the question, if there are more then these orbits in $\S 6, \S 8$, we investigate in the second part of $\S 5$ the $\mathfrak{G}$-action on $\tilde{\mathcal{M}}_{c}^{r e g}(n)$. Since for $(A, B, i, j)$ with $[A, B]=0$ the $G L(2)$-orbit of $i, j$ is not uniquely determined by the pair $(A, B)$ we only consider the $\mathfrak{G}$-action on the set of conjugation classes of so called stable pairs $(A, B)$, denoted by $\mathcal{S}(n)$, which are those pairs, that can occur in an ADHM datum. We show

Theorem 1.0.2 There are 2,3 respectively $6 \mathfrak{G}$-orbits in $\mathcal{S}(2), \mathcal{S}(3)$ and $\mathcal{S}(4)$.

In $\S 6$ we show that for a pair $(A, B)$ representing an element of $\mathcal{M}_{n c}^{\text {reg }}(n), n \leq 4$ there is no invariant subspace of $A$ and $B$, and investigate the spectral schemes for charge 2 and 3 . We prove that the spectral scheme of any element of $\mathcal{M}_{n c}^{r e g}(2)$ and $\mathcal{M}_{n c}^{r e g}(3)$ is reduced. Thus with Artamkins lemma we get

Theorem 1.0.3 There is one $\mathfrak{G}$-orbit in $\tilde{\mathcal{M}}_{n c}^{r e g}(2)$ and two orbits in $\tilde{\mathcal{M}}_{n c}^{\text {reg }}(3)$.

In $\S 7$ we sketch the proof of theorem 1.0.1 and show that the locally free sheaves corresponding to instantons are $G$-semistable. Because of this $G$-semistability we can associate in $\S 8$ so called coherent systems, introduced by LePotier in [LP1], to instantons of charge 4 and investigate with their help the spectral schemes. It turns out, that also any element of $\mathcal{M}_{n c}^{r e g}(4)$ has a reduced spectral scheme. Therefore we get

Theorem 1.0.4 There are $3 \mathfrak{G}$-orbits in $\tilde{\mathcal{M}}_{n c}^{r e g}(4)$. 


\section{Chapter 2}

\section{Tools and notations}

We give a short summery of definitions and facts about sheaves of modules on a smooth projective variety $X$ over $\mathbb{C}$, that we will need in this text.

\section{1 support, setsupport and Fitting scheme}

Let $X$ be of dimension $n$ and $\mathcal{G}$ a coherent sheaf on $X$. Then the rank of $\mathcal{G}$, denoted by $\operatorname{rg}(\mathcal{G})$ is the dimension of $\mathcal{G}_{\chi}$ over $\mathcal{O}_{X, \chi}$ at the generic point $\chi \in X$ (Considering $X$ as the associated integral scheme). The support of $\mathcal{G}$, denoted by $\operatorname{supp}(\mathcal{G})$ is the scheme, which restricted to an open affine set $U$ is given by $\operatorname{Spec}\left(\left.\mathcal{O}\right|_{U} / \operatorname{ann}\left(\left.\mathcal{G}\right|_{U}\right)\right)$, where $\left.\operatorname{ann}\left(\left.\mathcal{G}\right|_{U}\right) \subset \mathcal{O}\right|_{U}$ is the annihilator of $\left.\mathcal{G}\right|_{U}$. With $\operatorname{setsupp}(\mathcal{G})$ ) we denote the underlying set of $\operatorname{supp}(\mathcal{G})$. We say $\mathcal{G}$ is of dimension $d$ if $\operatorname{dim}(\operatorname{setsupp}(\mathcal{G}))=d$. Furthermore one can associate the following schemes to $\mathcal{G}$ :

For every point $x \in X$ there is a neighborhood $U$ of $x$ such that

$$
\left.\left.\mathcal{O}\right|_{U} ^{\oplus r} \stackrel{\phi}{\rightarrow} \mathcal{O}\right|_{U} ^{\oplus s} \rightarrow \mathcal{G} \rightarrow 0
$$

Let $\phi$ be given by a $r \times s$ matrix. Then the $k-t h$ Fitting scheme of $\mathcal{G}$ denoted by $\operatorname{Fitt}_{k}(\mathcal{G})$ is the scheme, that is locally the spectrum of the quotient of the structure sheaf of $X$ by the sheaf of ideals generated by the minors of $\phi$ of order $r-k$.

The advantage of $F_{i t t}(\mathcal{G})$ is that in opposite to $\operatorname{supp}(\mathcal{G})$ it "counts" the multiplicities of a sheaf in the right way. For example let $Y=\operatorname{Spec}\left(\mathbb{C}[x] / x^{2}\right)$ and consider the sheaves $\mathcal{O}_{Y}$ and $\mathcal{O}_{y} \oplus \mathcal{O}_{y}$, then $\operatorname{supp}\left(\mathcal{O}_{Y}\right)=Y$ and $\operatorname{supp}\left(\mathcal{O}_{y} \oplus \mathcal{O}_{y}\right)=$ $\left(y, \mathcal{O}_{y}\right)$ but both have $Y$ as their 0 -th Fitting scheme.

A scheme $S$ is called regular or nonsingular at a point $x$, if the dimension of the local ring $\mathcal{O}_{S, x}$ is equal to the dimension of the Zariski tangent space $T_{S, x}$ of $S$ 
at $x$. Note that in contrast to a variety the closed points of a scheme can be all singular. An example is $\operatorname{Spec}\left(\mathbb{C}[x, y] /\left(y^{2}\right)\right.$.

\subsection{Torsion sheaves}

For any open $U \subset X, \mathcal{G}(U)$ is an $\mathcal{O}(U)$ module. The torsion module of $\mathcal{G}(U)$ is given by

$$
T(\mathcal{G}(U)):=\{m \in \mathcal{G}(U): \exists a \in \mathcal{O}(U) \text { s.t } a \neq 0 \text { and } a \cdot m=0\}
$$

These torsion modules form a presheaf, whose sheafification is the torsion sheaf $\mathcal{T}(\mathcal{G})$ of $\mathcal{G}$. A coherent sheaf is called torsion free, if its torsion sheaf vanishes. $\mathcal{G}$ is a torsion sheaf if $\mathcal{T}(\mathcal{G})=\mathcal{G}$.

Since a coherent sheaf is uniquely determined by its restrictions to basic open subsets and the generic point of $X$ also belongs to the basic open subsets of $X$, the restrictions of a coherent sheaf of rank 0 to the basic open subsets are also of rank 0 . Therefore for a coherent subsheaf $\mathcal{F} \subset \mathcal{G}$ of rank 0 , one has

$$
\mathcal{F} \subset \mathcal{T}(\mathcal{G})
$$

Therefore a torsion free sheaf cannot have a subsheaf of rank 0 .

The dual of a coherent sheaf $\mathcal{G}$ is defined as $\mathcal{G}^{*}=\underline{\operatorname{Hom}}\left(\mathcal{G}, \mathcal{O}_{X}\right)$, thus on an open set $U \subset X$ one has $\mathcal{G}^{*}(U)=\operatorname{Hom}_{\mathcal{O}_{X}(U)}\left(\mathcal{G}(U), \mathcal{O}_{X}(U)\right)$. There is a natural homomorphism $\sigma_{\mathcal{G}(U)}: \mathcal{G}(U) \rightarrow \mathcal{G}^{* *}(U)$ given by

$$
\left(\sigma_{\mathcal{G}(U)}(m)\right)\left(g^{*}\right)=g^{*}(m) \forall g^{*} \in \mathcal{G}^{*}(U)
$$

and this maps extends to $\sigma_{\mathcal{G}}: \mathcal{G} \rightarrow \mathcal{G}^{* *}$. One has

$$
\operatorname{ker}\left(\sigma_{\mathcal{G}}\right)=\mathcal{T}(\mathcal{G})
$$

Therefore $\mathcal{G}$ is torsion free if and only if $\sigma_{\mathcal{G}}$ is injective. $\mathcal{G}$ is called reflexive if it is an isomorphism. The set of points, where a reflexive sheaf is not locally free is of codimension $\geq 3$, therefore a reflexive sheaf on a projective surface is already locally free. For more details see for example $[\mathrm{K}]$. 


\subsection{Duality, Ext and Ext}

Let $\mathcal{G}$ be a sheaf of modules on $X, \operatorname{Ext}^{i}(-, \mathcal{G})$ and $\underline{\operatorname{Ext}}{ }^{i}(-, \mathcal{G})$ be the right derived functors of $\operatorname{Hom}(-, \mathcal{G})$ and $\operatorname{Hom}(-, \mathcal{G})$ respectively. For the proof of the next lemma see $[\mathrm{H}] I I I .6,7$.

Lemma 2.3.1 Let $\mathcal{G}$ be sheaf of modules on $X$

1. $\operatorname{Ext}^{i}\left(\mathcal{O}_{X}, \mathcal{G}\right)=0$ for $i>0$;

2. $\operatorname{Ext}^{i}\left(\mathcal{O}_{X}, \mathcal{G}\right)=H^{i}(X, \mathcal{G})$ for $i \geq 0$;

3. For a locally free $\mathcal{L}$ and a sheaf of modules $\mathcal{F}$ one has

$$
\begin{gathered}
\operatorname{Ext}^{i}(\mathcal{G} \otimes \mathcal{L}, \mathcal{F}) \simeq \operatorname{Ext}^{i}\left(\mathcal{G}, \mathcal{F} \otimes \mathcal{L}^{*}\right) \\
\underline{\operatorname{Ext}} t^{i}(\mathcal{G} \otimes \mathcal{L}, \mathcal{F}) \simeq \underline{\operatorname{Ext}}\left(\mathcal{G}, \mathcal{F} \otimes \mathcal{L}^{*}\right) \simeq \underline{\operatorname{Ext}} t^{i}(\mathcal{G}, \mathcal{F}) \otimes \mathcal{L}^{*}
\end{gathered}
$$

4. (Duality for $\mathbb{P}_{n}$ ) For $X=\mathbb{P}_{n}$ and $\mathcal{G}$ coherent one has

$$
\operatorname{Ext}^{i}\left(\mathcal{G}, \omega_{\mathbb{P}_{n}}\right) \simeq H^{n-i}\left(\mathbb{P}_{n}, \mathcal{G}\right)^{*}
$$

where $\omega_{\mathbb{P}_{n}}=\mathcal{O}(-n-1)$ is the canonical sheaf of $\mathbb{P}_{n}$.

5. 2,3 and 4 together give

$$
H^{i}\left(\mathbb{P}_{n}, \mathcal{L}\right) \simeq H^{n-i}\left(\mathbb{P}_{n}, \mathcal{L}^{*} \otimes \omega_{\mathbb{P}_{n}}\right)^{*}
$$

6. If $Y$ is a closed subvariety of $\mathbb{P}_{n}$ of codimension $d$ and $\mathcal{F}$ a sheaf of $\mathcal{O}_{Y^{-}}$ modules, then

$$
\operatorname{Hom}_{Y}\left(\mathcal{F}, \omega_{Y}\right) \simeq \operatorname{Ext}_{\mathbb{P}_{n}}^{d}\left(\mathcal{F}, \omega_{\mathbb{P}_{n}}\right)
$$

7. For $x \in \mathbb{P}_{n}$ one has

$$
\underline{\operatorname{Ext}}^{i}(\mathcal{G}, \mathcal{F})_{x} \simeq \operatorname{Ext}_{\mathcal{O}_{x}}^{i}\left(\mathcal{G}_{x}, \mathcal{F}_{x}\right)
$$




\subsection{Torsion and duality for sheaves of rank 0}

The following can be found in [LP1] chapter 8 .

Lemma 2.4.1 Let $\mathcal{G}$ be a coherent sheaf of codimension $c$ on a smooth projective variety $X$ of dimension $n$. Then $\underline{\text {Ext}^{q}}\left(\mathcal{G}, \omega_{X}\right)=0$ for $q<c, \underline{\operatorname{Ext}} \boldsymbol{t}^{c}\left(\mathcal{G}, \omega_{X}\right)$ is of codimension $c$ and $\underline{\operatorname{Ext}} \underline{q}^{q}\left(\mathcal{G}, \omega_{X}\right)$ is of codimension $\geq q$ for $q>c$.

There is also a concept of torsion subsheaves and duality for sheaves of rank 0 : A sheaf is called torsion free on its support if there is no subsheaf of lower dimension. Setting $\mathcal{G}^{-}:=\underline{E x t^{c}}\left(\mathcal{G}, \mathcal{O}_{X}\right)$ for $\mathcal{G}$ of codimension $c$, one also has a canonical homomorphism $\mathcal{G} \rightarrow \mathcal{G}^{\sim}$, which is injective if $\mathcal{G}$ is torsion free on its support, and in case of an isomorphism $\mathcal{G}$ is called reflexive on its support. One has

Lemma 2.4.2 Let $\mathcal{G}$ be a coherent sheaf of codimension c on a smooth projective variety $X$ of dimension $n . \mathcal{G}$ is torsion free on its support if and only for all $q>c$ the support of $\underline{\operatorname{Ext}^{q}}\left(\mathcal{G}, \omega_{X}\right)$ is of codimension $\geq q+1$.

and

Lemma 2.4.3 Let $\mathcal{G}$ be a coherent sheaf of codimension c on a smooth projective variety $X$ of dimension $n . \mathcal{G}$ is reflexive on its support if and only for all $q>c$ the support of $\underline{\operatorname{Ext}}{ }^{q}\left(\mathcal{G}, \omega_{X}\right)$ is of codimension $\geq q+2$.

Therefore if $X$ is a surface and $\mathcal{G}$ is of codimension 1 and torsion free on its support one has

$$
\mathcal{G} \simeq \mathcal{G}^{\sim}
$$

\section{$2.5 \quad$ Further tools}

Let $\underline{\operatorname{Tor}}^{i}(-, \mathcal{G})$ be the $i$-th left derived functor of $-\otimes \mathcal{G}$. Considering the homological dimension of the stalk of a sheaf $\mathcal{G}$ on $X$, which is the length of the shortest free resolution, one can show using point 7 of lemma 2.3 .1 and a similar isomorphism for $\underline{\operatorname{Tor}}^{i}$ (see $[\mathrm{K}]$ )

Lemma 2.5.1 Let $\mathcal{G}$ be a torsion free sheaf on $X$. Then $\mathcal{G}$ is locally free at $x$ if and only if one of the following two equivalent conditions is satisfied:

1. $\operatorname{Tor}^{i}\left(\mathcal{G},\left.\mathcal{O}\right|_{x}\right)=0$ for $i>0$. 
2. $\underline{\operatorname{Ext}^{i}}\left(\mathcal{G}, \mathcal{O}_{X}\right)_{x}=0$ for $i>0$.

where $\left.\mathcal{O}\right|_{x}$ is the structure sheaf of the reduced scheme supported at $x$. The next two statements can be found for example in [OSS].

Theorem 2.5.2 (Theorem A of Serre) Let $\mathcal{G}$ be a coherent sheaf on $\mathbb{P}_{n}$. Then there is $k_{0} \in \mathbb{Z}$ such that $\mathcal{G}(k)$ is generated by global sections for $k \geq k_{0}$

Theorem 2.5.3 (Theorem B or vanishing theorem of Serre) Let $\mathcal{G}$ be a coherent sheaf on $\mathbb{P}_{n}$. Then there is a $k_{0} \in \mathbb{Z}$ such that for $q>0$ and $k \geq k_{0}$ one has

$$
H^{q}(\mathcal{G}(k))=0
$$

Furthermore we will use monads. A monad is a complex

$$
0 \rightarrow \mathcal{F} \stackrel{a}{\rightarrow} \mathcal{G} \stackrel{b}{\rightarrow} \mathcal{H} \rightarrow 0
$$

of sheaves, which is exact at $\mathcal{F}$ and $\mathcal{H}$. The sheaf $\mathcal{E}:=\operatorname{ker}(b) / \operatorname{im}(a)$ is called the cohomology of the monad.

\subsection{Grothendieck and Chern classes}

The Grothendieck group of a smooth projective algebraic variety $X$ of dimension $n$, denoted by $K(X)$, is the quotient of the free abelian group generated by all coherent sheaves on $X$ by the subgroup generated by short exact sequences. That is, an element $\mathcal{G}-\mathcal{G}^{\prime}-\mathcal{G}^{\prime \prime}$ vanishes in $K(X)$, if there is an exact sequence $0 \rightarrow \mathcal{G}^{\prime} \rightarrow \mathcal{G} \rightarrow \mathcal{G}^{\prime \prime} \rightarrow 0$. We denote the equivalence class of a sheaf $\mathcal{G}$ in $K(X)$ by $g(\mathcal{G})$ and call it the Grothendieck class of $\mathcal{G}$.

For $X=\mathbb{P}_{n}, K\left(\mathbb{P}_{n}\right)$ is freely generated by $k^{i}:=\left.\mathcal{O}\right|_{\mathbb{P}\left(L_{i}\right)} ; i=0,1, \ldots, n$ as abelian group, where $L_{i}$ is a linear subspace of $\mathbb{C}^{n+1}$ of codimension $i$. Thus we write the Grothendieck class of a coherent sheaf $\mathcal{G}$ on $\mathbb{P}_{n}$ as

$$
g(\mathcal{G})=\sum_{i=0}^{n} g_{i}(\mathcal{G}) k^{i}
$$

The Grothendieck group also has a ring structure given by

$$
g \cdot \tilde{g}=\sum_{i=0}^{n}\left(\sum_{j+m=i} g_{j} \tilde{g}_{m}\right) k^{i}
$$

Considering it with this additional structure we call it the Grothendieck ring. 
Definition 2.6.1 For a coherent sheaf $\mathcal{G}$ on $\mathbb{P}_{n}$ of codimension d the multiplicity of $\mathcal{G}$ is given by

$$
m(\mathcal{G}):=g_{d}(\mathcal{G})
$$

The Chow ring $A(X)$ is the graded object

$$
A(X)=\bigoplus_{i=0}^{n} A^{i}(X)
$$

where $A^{i}(X)$ is the set of cycles $\sum m_{j} Y_{j}$ with $Y_{j}$ irreducible subvarieties of $X$ of codimension $j$ and $m_{j} \in \mathbb{Z}$ modulo rational equivalence (see $[\mathrm{H}] \mathrm{p}$. 426). It comes with a map $A^{r} \times A^{s} \rightarrow A^{r+s}$ which generalizes the intersection of two divisors on a surface and implements a ring structure on $A(X)$.

The Chow ring of $\mathbb{P}_{n}, A\left(\mathbb{P}_{n}\right)$, as a group is freely generated by $n+1$ elements $1, h, h^{2}, \cdots h^{n}$ :

$$
A\left(\mathbb{P}_{n}\right)=\mathbb{Z}[h] / h^{n+1},
$$

where $h$ is the Chow class of a hyperplane. The Chern character of a coherent sheaf $\mathcal{G}$ on $\mathbb{P}_{2}$, which is an element of $A\left(\mathbb{P}_{2}\right)$, is given by

$$
\operatorname{ch}(\mathcal{G})=r k(\mathcal{G})+c_{1}(\mathcal{G}) h+\frac{1}{2}\left(c_{1}(\mathcal{G})^{2}-2 c_{2}(\mathcal{G})\right) h^{2}
$$

and with Hirzebruch-Riemann-Roch one gets

$$
\chi(E)=r k(\mathcal{G})+\frac{3}{2} c_{1}(\mathcal{G})+\frac{1}{2}\left(c_{1}(\mathcal{G})^{2}-2 c_{2}(\mathcal{G})\right)
$$

We compute the Chern characters of the generators of $K\left(\mathbb{P}_{2}\right)$ :

$\mathcal{O}(i)$ : With 2.6.1 we have

$$
\operatorname{ch}(\mathcal{O}(i))=1+i h+\frac{i^{2}}{2} h^{2}
$$

$\left.\mathcal{O}\right|_{l}(i)$ : We consider the resolution of $\left.\mathcal{O}\right|_{l}(i)$ :

$$
\left.0 \rightarrow \mathcal{O}(i-1) \rightarrow \mathcal{O}(i) \rightarrow \mathcal{O}\right|_{l}(i) \rightarrow 0
$$


and get

$$
\operatorname{ch}\left(\left.\mathcal{O}\right|_{l}(i)\right)=\operatorname{ch}(\mathcal{O}(i))-\operatorname{ch}(\mathcal{O}(i-1))=h+\frac{2 i-1}{2} h^{2}
$$

$\left.\mathcal{O}\right|_{p}$ : There is the resolution:

$$
\left.\left.\left.0 \rightarrow \mathcal{O}\right|_{l}(-1) \rightarrow \mathcal{O}\right|_{l} \rightarrow \mathcal{O}\right|_{p} \rightarrow 0
$$

Thus $\operatorname{ch}\left(\left.\mathcal{O}\right|_{p}\right)=h^{2}$.

Notation: Since we will not be working with the local ring at a point $p$ in this text, we will often denote $\left.\mathcal{O}\right|_{p}$ by $\mathcal{O}_{p}$.

The image of a generic element of $K\left(\mathbb{P}_{2}\right)$ is

$$
\operatorname{ch}(g)=\operatorname{ch}\left(g_{0}+g_{1} k+g_{2} k^{2}\right)=g_{0}+g_{1} h+\left(g_{2}-\frac{g_{1}}{2}\right) h^{2}
$$

This formula shows, that $g_{1}=c_{1}$ for a sheaf on $\mathbb{P}_{2}$, therefore for a sheaf $\theta$ of codimension 1 on $\mathbb{P}_{2}$ one has $m(\theta)=c_{1}(\theta)$.

We compute the Hilbert polynomial of a coherent sheaf $\mathcal{G}$ on $\mathbb{P}_{2}$. By definition $P_{\mathcal{G}}(n)=\chi(\mathcal{G}(n))$. To apply 2.6.2, we need the Chern classes of the twisted sheaf $\mathcal{G}(n)$ in terms of the Chern classes of $\mathcal{G}$ : One has

$$
\begin{aligned}
& \operatorname{ch}(\mathcal{G}(n))=\operatorname{ch}(\mathcal{G}) \operatorname{ch}(\mathcal{O}(n))= \\
= & \left(r k(\mathcal{G})+c_{1}(\mathcal{G}) h+\frac{1}{2}\left(c_{1}(\mathcal{G})^{2}-2 c_{2}(\mathcal{G})\right) h^{2}\right)\left(1+n h+\frac{1}{2} n^{2} h^{2}\right)= \\
= & r k(\mathcal{G})+\left(c_{1}(\mathcal{G})+r k(\mathcal{G}) n\right) h+\left(\frac{1}{2} c_{1}(\mathcal{G})^{2}-c_{2}(\mathcal{G})+\frac{1}{2} r k(\mathcal{G}) n^{2}+c_{1}(\mathcal{G}) n\right) h^{2}
\end{aligned}
$$

Thus $c_{1}(\mathcal{G}(n))=c_{1}(\mathcal{G})+r k(\mathcal{G}) n$ and

$$
\begin{aligned}
& \frac{1}{2} c_{1}(\mathcal{G}(n))^{2}-c_{2}(\mathcal{G}(n))=\frac{1}{2} c_{1}(\mathcal{G})^{2}+r k(\mathcal{G}) c_{1}(\mathcal{G}) n+\frac{1}{2} r k(\mathcal{G})^{2} n^{2}-c_{2}(\mathcal{G}(n))= \\
= & \frac{1}{2} c_{1}(\mathcal{G})^{2}-c_{2}(\mathcal{G})+\frac{1}{2} r k(\mathcal{G}) n^{2}+c_{1}(\mathcal{G}) n
\end{aligned}
$$

giving $2 c_{2}(\mathcal{G}(n))=r k(\mathcal{G})(r k(\mathcal{G})-1) n^{2}+2(r k(\mathcal{G})-1) c_{1}(\mathcal{G}) n+2 c_{2}(\mathcal{G})$

Using this with 2.6.2 gives:

$$
\begin{array}{rlrl}
P_{\mathcal{G}}(n) & =r k(\mathcal{G})+\frac{3}{2} c_{1}(\mathcal{G})+\frac{3}{2} r k(\mathcal{G}) n+\frac{1}{2}\left(c_{1}(\mathcal{G})^{2}-2 c_{2}(\mathcal{G})\right)+\frac{1}{2} r k(\mathcal{G}) n^{2}+c_{1}(\mathcal{G}) n \\
& = & \frac{1}{2} r k(\mathcal{G}) n^{2}+\left(\frac{3}{2} r k(\mathcal{G})+c_{1}(\mathcal{G})\right) n+\chi(\mathcal{G})
\end{array}
$$




\subsection{The Grothendieck spectral sequence}

A (cohomology) spectral sequence is a sequence of double complexes $E_{r}^{p q}, r \in \mathbb{N}$ with differentials

$$
d_{r}^{p q}: E_{r}^{p q} \rightarrow E_{r}^{p+r, q-r+1}
$$

such that $E_{r+1}^{p q}$ is the cohomology of $\left(E_{r}^{p q}, d_{r}^{p q}\right)$. We say $E_{r}^{p q}$ is bounded if for any $n \in \mathbb{Z}$ there is a $r_{0}$ such that for all $p, q$ with $p+q=n$ one has $E_{r_{0}}^{p q}=E_{r_{0}+j}^{p, q}=: E_{\infty}^{p q}$ for $j \in \mathbb{N}$. We say $E_{r}^{p q}$ converges to $H^{*}$ in any object $H^{n}$ has a filtration

$0=F^{t} H^{n} \subseteq \cdots F^{p+1} H^{n} \subseteq F^{p} H^{n} \cdots \subseteq F^{s} H^{n}=H^{n}$ s.t $E_{\infty}^{p q} \simeq F^{p} H^{p+q} / F^{p+1} H^{p+q}$

In this case we write $E_{r}^{p q} \Rightarrow H^{p+q}$.

Now we come to the Grothendieck spectral sequence. Let $\mathcal{A}, \mathcal{B}, \mathcal{C}$ be abelian categories with $\mathcal{A}, \mathcal{B}$ having enough injectives and left exact functors $G: \mathcal{A} \rightarrow$ $\mathcal{B} ; F: \mathcal{B} \rightarrow \mathcal{C}$ such that $G$ sends injective objects of $\mathcal{A}$ to acyclic objects of $\mathcal{B}$ (that is $R^{i} F(G(a))=0$ for $i>0$ and $a$ any injective object), then for each object $a \in \mathcal{A}$ there is a spectral sequence with

$$
E_{2}^{p q}=\left(R^{p} F\right)\left(R^{q} G\right)(a) \Rightarrow R^{p+q}(F G)(a)
$$

The maps

$$
R^{p}(F)(G(a)) \rightarrow R^{p}(F G)(a) \text { and } R^{q}(F G)(a) \rightarrow F\left(R^{q} G(a)\right)
$$

are natural transformations. Furthermore one has the so called exact sequence of low degree

$$
0 \rightarrow\left(R^{1} F\right)(G(a)) \rightarrow R^{1}(F G)(a) \rightarrow F\left(R^{1} G(a)\right) \rightarrow\left(R^{2} F\right)(G(a)) \rightarrow R^{2}(F G)(a)
$$




\section{Chapter 3}

\section{The action of the group of biregular automorphism of the affine plane on instantons}

We give three equivalent descriptions of $\mathcal{M}^{\text {reg }}(n)$, the space of framed regular $S U(2)$ instantons of charge $n$ over $\mathbb{R}^{4}$ and define an action of the group of biregular automorphism of $\mathbb{C}^{2}$ on $\mathcal{M}^{\text {reg }}(n)$.

It is known [Sh1], that the group of biregular automorphism of the complex affine plane, which we denote by $\mathfrak{G}$, is the amalgamated product

$$
\mathfrak{G}=\mathcal{A}_{2} *_{\Gamma} \mathfrak{E}
$$

where the subgroups $\mathcal{A}_{2}, \Gamma, \mathfrak{E}$ are given by

$$
\begin{gathered}
\mathcal{A}_{2}=\left\{(x, y) \rightarrow\left(\mu_{0} x+\mu_{1} y+\mu_{2}, \nu_{0} x+\nu_{1} y+\nu_{2}\right) \mid \operatorname{det}(a)=\operatorname{det}\left(\begin{array}{cc}
\mu_{0} & \mu_{1} \\
\nu_{0} & \nu_{1}
\end{array}\right) \neq 0\right\} \\
\mathfrak{E}=\{(x, y) \mapsto(x, y+p(x)) \mid p \in \mathbb{C}[x]\}, \quad \Gamma=A_{2} \cap \mathfrak{E} .
\end{gathered}
$$

The following actions of $\mathcal{A}_{2}$ and $\mathfrak{E}$ canonically induce an action of $\mathcal{G}$ on the set of pairs of matrices. For $(A, B) \in g l(n) \times g l(n), a \in \mathcal{A}_{2}$ and $e_{p} \in \mathfrak{E}$ let

$$
\begin{gathered}
a(A, B)=\left(\mu_{0} A+\mu_{1} B+\mu_{2} \mathbf{1}, \nu_{0} A+\nu_{1} B+\nu_{2} \mathbf{1}\right), \\
e_{p}(A, B)=(A, B+p(A)) .
\end{gathered}
$$


We want to extend this action to an action of $\mathfrak{G}$ on framed instantons, these are anti self dual $S U(2)$-connections on bundles over $\mathbb{R}^{4}$. Their original description by ADHM data, as it can be found in [DK], states that the space of these connections with charge $n$ can be described as

$$
\mathcal{M}^{r e g}(n)=\left\{\begin{array}{ll} 
& (i)[A, B]+i j=0 \\
(A, B, i, j) \mid & (i i)\left[A, A^{*}\right]+\left[B, B^{*}\right]+i i^{*}-j^{*} j=0 \\
& (i i i) \operatorname{Stab}(n) \\
(A, B, i, j)=\mathbf{1}
\end{array}\right\} / U(n)
$$

where $A, B \in \operatorname{End}(V), i \in \operatorname{Hom}(W, V)$ and $j \in \operatorname{Hom}(V, W)$ with $V$ and $W$ hermitian vector spaces of dimension $n$ and 2 respectively and $U(n)$-action, which is given by the $G L(n)$-action

$$
g(A, B, i, j)=\left(g^{-1} A g, g^{-1} B g, g^{-1} i, j g\right) \text { for } g \in G L(n)
$$

Definition 3.0.1 We call a tuple $(A, B, i, j)$ stable if it satisfies the following conditions

(S1) $\nexists$ proper subspace $S \subset V$ such that $A(S) \subset S ; B(S) \subset S$ and $i m(i) \subset S$

(S2) \# proper subspace $S \subset V$ such that $A(S) \subset S ; B(S) \subset S$ and $S \subset k e r(j)$

Remark: $(A, B, i, j) \mapsto\left(B^{t}, A^{t}, j^{t}, i^{t}\right)$ is an involution on the set of tuples $(A, B, i, j)$. It preserves stability. For a tuple $(A, B, i, j)$ the condition $(\mathbf{S} 1)$ respectively (S2) is violated if and only if the condition (S2) respectively (S1) is violated for $\left(B^{t}, A^{t}, j^{t}, i^{t}\right)$. Therefore to show stability of $(A, B, i, j)$ it suffices to show condition $(\mathbf{S 1})$ for $(A, B, i, j)$ and $\left(B^{t}, A^{t}, j^{t}, i^{t}\right)$.

For the proof of the next lemma we will need a lemma of Mumford [MFK]

Lemma 3.0.2 Let $G$ be a finite dim. Lie group acting symlecticly on a symplectic manifold $X$ with moment map $\mu: X \rightarrow \mathfrak{g}^{*}$. Suppose $\mu(x)=0$ for an $x \in X$ and $\operatorname{Stab}_{G}(x)=1$. Then for the action of $G^{\mathbb{C}}$ induced by the action of $G$ one has $\operatorname{Stab}_{G^{\mathbb{C}}}(x)=\mathbf{1}$. 
Lemma 3.0.3 $\mathcal{M}^{\text {reg }}(n)$ is bijective to each of the following two set

$$
\begin{aligned}
& \left\{\begin{array}{cl} 
& (i)[A, B]+i j=0 \\
(A, B, i, j) \mid & (i i) G L(n)(A, B, i, j) \text { is closed } \\
& (i i i) S t a b_{G L(n)}(A, B, i, j)=\mathbf{1}
\end{array}\right\} / G L(n) ; \\
& \left\{\begin{array}{ll}
(A, B, i, j) \mid & (A, B]+i j=0 \\
(i i)(A, B, i, j) \text { is stable }
\end{array}\right\} / G L(n) .
\end{aligned}
$$

\section{Proof:}

Consider the vector space $\mathcal{V}$ of tuples $(A, B, i, j)$ with the hermitian metric induced from the metrics on $V$ and $W$ and canonical symplectic form. The $U(n)$ action given by

$$
u(A, B, i, j)=\left(u^{-1} A u, u^{-1} B u, u^{-1} i, j u\right)
$$

is symplectic. The corresponding moment map is

$$
\mu: \mathcal{V} \rightarrow \mathfrak{u}(n)^{*} ; \quad(A, B, i, j) \mapsto\left[A, A^{*}\right]+\left[B, B^{*}\right]+i i^{*}-j^{*} j
$$

The Lie group $U(n)$ is semisimple and its complexification is $G L(n)$. By a theorem of Kempf and Ness ([N], Th.3.12) there is a bijection

$$
\mu^{-1}(0) / U(n) \rightarrow\{\text { closed } G L(n) \text {-orbits in } \tilde{V}\}
$$

Since condition $(i i)$ of 3.0.1 for an ADHM datum is $\mu(A, B, i, j))=0$, we get $(i i)$ of 3.0.2 by applying 3.0.4, applying lemma 3.0.2 to $U(n)$ gives (iii) of 3.0.2 thus we have shown the first equivalence.

The Hilbert-Mumford criterion states that every point in the closure of an $G L(n)$ orbit $\mathcal{O}$ is in the closure of a 1-parameter family, we use this to show the equivalence of 3.0.2 and 3.0.3.

Let $G L(n)(A, B, i, j)$ be closed and $\operatorname{Stab}_{G L(n)}(A, B, i, j)=\mathbf{1}$. Assume $(A, B, i, j)$ does not satisfy (S1) then $\exists S \subset V$ such that $V=S \oplus S^{\perp}$ with

$$
A=\left(\begin{array}{cc}
* & * \\
0 & *
\end{array}\right) \quad B=\left(\begin{array}{cc}
* & * \\
0 & *
\end{array}\right) \quad i=\left(\begin{array}{c}
* \\
0
\end{array}\right) .
$$

Choose $\lambda(t):=\left(\begin{array}{cc}\mathbf{1} & 0 \\ 0 & t^{-1} \mathbf{1}\end{array}\right)$, then 


$$
\begin{array}{rlr}
\lim _{t \rightarrow 0} A^{\lambda(t)}=\left(\begin{array}{cc}
* & 0 \\
0 & *
\end{array}\right), & \lim _{t \rightarrow 0} B^{\lambda(t)}=\left(\begin{array}{cc}
* & 0 \\
0 & *
\end{array}\right), \\
\lim _{t \rightarrow 0} \lambda(t) i=\left(\begin{array}{l}
* \\
0
\end{array}\right), & \lim _{t \rightarrow 0} j \lambda(t)^{-1}=\left(\begin{array}{cc}
* & 0
\end{array}\right) .
\end{array}
$$

Thus $g=\left(\begin{array}{cc}\mathbf{1} & 0 \\ 0 & \tau \mathbf{1}\end{array}\right) \in \operatorname{Stab}_{G L(n)}\left(\lim _{t \rightarrow 0} \lambda(t)(A, B, i, j)\right) \neq \mathbf{1} \Rightarrow$ $\operatorname{Stab}_{G L(n)}(A, B, i, j) \neq \mathbf{1}$, which is a contradiction thus $(A, B, i, j)$ must satisfy (S1).

Since $(A, B, i, j) \in \mathcal{M}^{\text {reg }}(n)$ if and only if $\left(B^{t}, A^{t}, j^{t}, i^{t}\right) \in \mathcal{M}^{\text {reg }}(n)$, and (S1) for $\left(B^{t}, A^{t}, j^{t}, i^{t}\right)$ is $(\mathbf{S 2})$ for $(A, B, i, j)$, the conditions " $G L(n)(A, B, i, j)$ is closed" and $\operatorname{Stab}_{G L(n)}(A, B, i, j)=\mathbf{1}$ also induce $(\mathbf{S 2})$.

Let $(A, B, i, j)$ be stable. Suppose $G L(n)(A, B, i, j)$ is not closed, then by the Hilbert-Mumford criterion there exists a 1-parameter family $\lambda: \mathbb{C}^{*} \rightarrow G L(n)$ such that $\lim _{t \rightarrow 0} \lambda(t)(A, B, i, j)$ is not in $G L(n)(A, B, i, j)$. Consider the weight decomposition of $V$ with respect to $\lambda$, which has real weights only: $V=$ $\bigoplus_{\alpha} V(\alpha)$. Because of the existence of the limit we must have

$$
\begin{gathered}
A(V(\alpha)) \subset \bigoplus_{\beta \geq \alpha} V(\beta), \quad B(V(\alpha)) \subset \bigoplus_{\beta \geq \alpha} V(\beta), \\
i m(i) \subset \bigoplus_{\alpha \geq 0} V(\beta), \quad \bigoplus_{\alpha>0} V(\alpha) \subset \operatorname{ker}(j) .
\end{gathered}
$$

If there is a positive weight, then with $S=V(\alpha)$, where $\alpha$ is the highest weight, (S2) is violated and therefore $(A, B, i, j)$ would not be stable.

If there is a negative weight, then with $S=\bigoplus_{\alpha \geq 0} V(\beta)$ (S1) would be violated and the tuple therefore not stable.

Thus $\lambda(t)$ can only have the weight 0 , but that means $\lambda(t)=$ const, and that contradicts that $\lim _{t \rightarrow 0} \lambda(t)(A, B, i, j)$ is not in $G L(n)(A, B, i, j)$. Thus stability induces a closed orbit.

Finally we show, that stability also induces a trivial stabilizer: Suppose $(A, B, i, j)$ is stable but $\operatorname{Stab}_{G L(n)}(A, B, i, j) \neq \mathbf{1}$. Then let $s \in$ $\operatorname{Stab}_{G L(n)}(A, B, i, j)$. We have to consider two cases:

1. $V$ is a generalized eigenspace of $s$. Then $s^{-1} i=i$ and $j s=j$ imply $i=j=0$. That means $[A, B]=0$, therefore it exists an at least one dimensional common eigenspace $S$ of $A$ and $B$. This $S$ violates (S2). 
2. Otherwise let $V_{1}$ be a generalized eigenspace of $S$, which is not an eigenspace, or an eigenspace with eigenvalue $\neq 1$ which is also a generalized eigenspace. Then with $V=V_{1} \oplus V_{2}$ and a basis of $V$ such that $s$ has Jordan normal form one computes that $s \in \operatorname{Stab}_{G L(n)}(A, B, i, j)$ implies

$$
A, B=\left(\begin{array}{cc}
* & * \\
0 & *
\end{array}\right), \quad j=\left(\begin{array}{ll}
0 & *
\end{array}\right)
$$

Thus $V_{1}$ violates (S2).

This completes the proof.

Lemma 3.0.4 The following actions of $\mathcal{A}_{2}$ and $\mathfrak{E}$ on $\mathcal{M}^{\text {reg }}(n)$ are well defined and induce therefore a $\mathfrak{G}$-action on $\mathcal{M}^{\text {reg }}(n)$.

$$
\begin{gathered}
a(A, B, i, j)=\left(\mu_{0} A+\mu_{1} B+\mu_{2} \mathbf{1}, \nu_{0} A+\nu_{1} B+\nu_{2} \mathbf{1}, \operatorname{det}(a) i, j\right) \\
e(A, B, i, j)=(A, B+p(A), i, j)
\end{gathered}
$$

\section{Proof:}

We use 3.0.3.

(i) This action, which is defined on $\mathcal{V}$, commutes with the $G L(n)$-action. The $\mathfrak{G}$-action therefore descends to $\mathcal{V} / G L(n)$.

(ii) One has

$$
\begin{aligned}
{\left[\mu_{0} A+\mu_{1} B+\right.} & \left.\mu_{2} \mathbf{1}, \nu_{0} A+\nu_{1} B+\nu_{2} \mathbf{1}\right]+\operatorname{det}(a) i j= \\
=\left(\mu_{0} \nu_{1}-\mu_{1} \nu_{0}\right)[A, B]+\operatorname{det}(a) i j & =\operatorname{det}(a)([A, B]+i j)=0,
\end{aligned}
$$

and

$$
[A, B+p(A)]+i j=[A, B]+i j=0 .
$$

Therefore the $\mathfrak{G}$-action preserves condition (i) of 3.0.3. 
(iii) Suppose $a(A, B, i, j)$ is not stable. Then there exists $S \subsetneq V$ such that

$$
\left(\mu_{0} A+\mu_{1} B\right)(S) \subset S \quad\left(\nu_{0} A+\nu_{1} B\right)(S) \subset S
$$

Therefore

$$
\frac{1}{\operatorname{det}(a)}\left(\nu_{1}\left(\mu_{0} A+\mu_{1} B\right)-\mu_{1}\left(\nu_{0} A+\nu_{1} B\right)\right)(S)=A(S) \subset S,
$$

and one shows in the same way that $B(S) \subset S$. Thus if $a(A, B, i, j)$ is not stable then $(A, B, i, j)$ is not stable, which means that the action of $\mathcal{A}(2)$ preserves condition $(i i)$.

If $e_{p}(A, B, i, j)$ is not stable, then there exists $S \subsetneq V$ such that

$$
A(S) \subset S \quad(B+p(A))(S) \subset S
$$

But $A(S) \subset S$ implies $p(A)(S) \subset S$, and therefore $B(S)=(B+p(A)-$ $p(A))(S) \subset S$. Thus the $\mathfrak{G}$-action preserves condition (ii) of 3.0.3.

This completes the proof. 


\section{Chapter 4}

\section{Instantons as vector bundles on $\mathbb{P}_{2}$}

Now we will show, that there is a 1-to-1 correspondence between $\mathcal{M}^{\text {reg }}(n)$ and the set of locally free sheaves of rank 2 on $\mathbb{P}_{2}$ with $\left(c_{1}, c_{2}\right)=(0, n)$, which are trivial on a fixed line $l_{\infty} \subset \mathbb{P}_{2}$. We start with

Theorem 4.0.5 (Nakajima $[N]$ )

Let $\mathfrak{M}(n)$ be the set of torsion free sheaves $\mathcal{F}$ of rank 2 on $\mathbb{P}_{2}$ with $\left(c_{1}(\mathcal{F}), c_{2}(\mathcal{F})\right)=(0, n)$, trivial at $l_{\infty}$ with framing of $H^{0}\left(\left.\mathcal{F}\right|_{l_{\infty}}\right)$. There is a bijection of $\mathfrak{M}(n)$ to

$$
\left\{\begin{array}{ll}
(A, B, i, j) \mid & (i)[A, B]+i j=0 \\
& (i i)(A, B, i, j) \text { satisfies }(\mathbf{S 1})
\end{array}\right\} / G L(n)
$$

Thus using this bijection and 3.0.3 we can identify

$$
\mathcal{M}^{r e g}(n) \subset \mathfrak{M}(n)
$$

with "trivial at $l_{\infty}$ " we mean that $\mathcal{F}$ is locally free at any $p \in l_{\infty}$ and that $\left.\mathcal{F}\right|_{l_{\infty}}=\mathcal{O}_{l_{\infty}} \oplus \mathcal{O}_{l_{\infty}}$

We divide the proof into a couple of lemmata.

Lemma 4.0.6 Let $\mathcal{F}$ be a torsion free sheaf of rank 2 on $\mathbb{P}_{2}$ with $\left(c_{1}, c_{2}\right)=(0, n)$ trivial at $l_{\infty}$ then

$$
\text { 1. } H^{0}(\mathcal{F}(-1))=H^{0}(\mathcal{F}(-2))=0
$$


2. $H^{2}(\mathcal{F}(-1))=H^{2}(\mathcal{F}(-2))=0$

3. $H^{0}(\mathcal{F}(-1) \otimes \Omega(1))=H^{2}(\mathcal{F}(-1) \otimes \Omega(1))=0$

\section{Proof:}

We consider the resolution of $\mathcal{O}_{l_{\infty}}$ :

$$
0 \rightarrow \mathcal{O}(-1) \rightarrow \mathcal{O} \rightarrow \mathcal{O}_{l_{\infty}} \rightarrow 0
$$

We tensor it with $\mathcal{F}(-k)$ : Since $\underline{\operatorname{Tor}}^{1}(\mathcal{F}(-k), \mathcal{O})=0$ and $\mathcal{F}$ is torsion free one has $\underline{\operatorname{Tor}}^{1}\left(\mathcal{F}, \mathcal{O}_{l_{\infty}}\right)=0$, thus we get

$$
\left.0 \rightarrow \mathcal{F}(-k-1) \rightarrow \mathcal{F}(-k) \rightarrow \mathcal{F}\right|_{l_{\infty}}(-k) \rightarrow 0
$$

The resulting long exact sequence of cohomology is

$$
\begin{aligned}
& 0 \rightarrow H^{0}(\mathcal{F}(-k-1)) \rightarrow H^{0}(\mathcal{F}(-k)) \rightarrow H^{0}\left(\left.\mathcal{F}\right|_{l_{\infty}}(-k)\right) \rightarrow \\
& \rightarrow H^{1}(\mathcal{F}(-k-1)) \rightarrow H^{1}(\mathcal{F}(-k)) \rightarrow H^{1}\left(\left.\mathcal{F}\right|_{l_{\infty}}(-k)\right) \rightarrow \\
& \rightarrow H^{2}(\mathcal{F}(-k-1)) \rightarrow H^{2}(\mathcal{F}(-k)) \rightarrow \quad 0
\end{aligned}
$$

Now

$$
\begin{gathered}
H^{1}\left(\mathbb{P}_{2},\left.\mathcal{F}\right|_{l_{\infty}}(-k)\right)^{*} \cong \operatorname{Ext}_{\mathbb{P}_{2}}^{1}\left(\left.\mathcal{F}\right|_{l_{\infty}}(-k), \omega_{\mathbb{P}_{2}}\right) \cong \\
\cong \operatorname{Hom}_{l_{\infty}}\left(\left.\mathcal{F}\right|_{l_{\infty}}(-k), \omega_{l_{\infty}}\right) \cong H^{0}\left(l_{\infty}, \mathcal{O}_{l_{\infty}}(k-2) \oplus \mathcal{O}_{l_{\infty}}(k-2)\right)
\end{gathered}
$$

Thus $H^{1}\left(\left.\mathcal{F}\right|_{l}(-k)\right)=0$ for $k<2$. Therefore 4.0 .2 gives

$$
H^{2}(\mathcal{F}(-k-1)) \cong H^{2}(\mathcal{F}(-k)) \text { for } k<2
$$

Now Serres vanishing theorem says that there is a $j_{0} \in \mathbb{Z}$ such that $\forall j>j_{0}$ $H^{2}(\mathcal{F}(j))=0$, therefore by 4.0 .3

$$
H^{2}(\mathcal{F}(k))=0 \text { for } k \geq-2
$$

which is 2. This is also true for $\left(\mathcal{F}^{* *}\right)^{*}=\mathcal{F}^{*}$. But since $\mathcal{F}^{* *}$ is locally free we can use Serre duality for it: 


$$
H^{0}\left(\mathcal{F}^{* *}(-k)\right) \cong H^{2}\left(\mathcal{F}^{*}(k-3)\right)^{*}
$$

Together with the natural inclusion $H^{0}(\mathcal{F}(-k)) \hookrightarrow H^{0}\left(\mathcal{F}^{* *}(-k)\right)$ we get 1 .

To show 3. we tensor the resolution of $\mathcal{O}_{l_{\infty}}$ with $\mathcal{F}(-k) \otimes \Omega(1)$. The resulting long exact sequence of cohomology is

$$
\begin{aligned}
& 0 \rightarrow H^{0}(\mathcal{F}(-k-1) \otimes \Omega(1)) \rightarrow H^{0}(\mathcal{F}(-k) \otimes \Omega(1)) \quad \rightarrow \\
& \rightarrow H^{0}\left(\left.\mathcal{F}(-k) \otimes \Omega(1)\right|_{l_{\infty}}\right) \quad \rightarrow \quad H^{1}(\mathcal{F}(-k-1) \otimes \Omega(1)) \rightarrow \\
& \rightarrow \quad H^{1}(\mathcal{F}(-k) \otimes \Omega(1)) \quad \rightarrow \quad H^{1}\left(\left.\mathcal{F}(-k) \otimes \Omega(1)\right|_{l_{\infty}}\right) \quad \rightarrow \\
& \rightarrow H^{2}(\mathcal{F}(-k-1) \otimes \Omega(1)) \rightarrow H^{2}(\mathcal{F}(-k) \otimes \Omega(1)) \quad \rightarrow \quad 0
\end{aligned}
$$

The restriction of the tangent sheaf $\mathcal{T}_{\mathbb{P}_{2}}$ to any line (in particular to $l_{\infty}$ ) splits as follows (see [OSS] p.27)

$$
\left.\mathcal{T}_{\mathbb{P}_{2}}\right|_{l_{\infty}}=\mathcal{O}_{l_{\infty}}(2) \oplus \mathcal{O}_{l_{\infty}}(1)
$$

Therefore with $\underline{\operatorname{Ext}}{ }^{1}\left(\mathcal{O}_{l_{\infty}}(i), \mathcal{O}\right)=\mathcal{O}_{l_{\infty}}(1-i)$ we get

$$
\begin{aligned}
& \left.\Omega(1)\right|_{l_{\infty}} \quad=\quad \Omega \otimes \mathcal{O}_{l_{\infty}}(1) \quad=\Omega \otimes \underline{\operatorname{Ext}^{1}}\left(\mathcal{O}_{l_{\infty}}, \mathcal{O}\right)= \\
& =\underline{\operatorname{Ext}^{1}}\left(\mathcal{T}_{l_{\infty}}, \mathcal{O}\right)=\underline{\operatorname{Ext}^{1}}\left(\mathcal{O}_{l_{\infty}}(2) \oplus \mathcal{O}_{l_{\infty}}(1), \mathcal{O}\right)=\mathcal{O}_{l_{\infty}} \oplus \mathcal{O}_{l_{\infty}}(-1)
\end{aligned}
$$

With this we find $H^{1}\left(\left.(\mathcal{F}(-k) \otimes \Omega(1))^{*}\right|_{l_{\infty}}\right)=0$ for $k<1$ and 4.0 .4 gives

$$
H^{2}(\mathcal{F}(k-1) \otimes \Omega(1)) \cong H^{2}(\mathcal{F}(k) \otimes \Omega(1)) \text { for } k>-1
$$

which gives with Serres vanishing theorem $H^{2}(\mathcal{F}(-1) \otimes \Omega(1))=0$. Since this is also true for $(\mathcal{F}(-1) \otimes \Omega(1))^{* *}$ we get with Serre duality for this locally free sheaf and the natural inclusion of a sheaf into its double dual 3.

This completes the proof.

We will use this result in an application of a Beilinson spectral sequence, which is

Theorem 4.0.7 [OSS] Let $\mathcal{F}$ be a torsion free sheaf of rank $r$ over $\mathbb{P}_{2}$, then there is a spectral sequence with

$$
G_{1}^{p . q}=H^{q}\left(\mathcal{F} \otimes \Omega^{-p}(-p)\right) \otimes \mathcal{O}(p)
$$

which converges to $\mathcal{F}$ in degree 0 and to 0 otherwise. 
Lemma 4.0.8 Let $V$ and $\tilde{V}$ be vector spaces of dimension $n$ and $W$ a vector space of dimension $2 n+2$. There is a bijection from $\mathfrak{M}(n)$ to the set of pairs $(M, F r(\mathcal{F}))$, where $M$ is a monad of the form

$$
0 \rightarrow V \otimes \mathcal{O}(-1) \stackrel{a}{\rightarrow} W \otimes \mathcal{O} \stackrel{b}{\rightarrow} \tilde{V} \otimes \mathcal{O}(1) \rightarrow 0
$$

$\mathcal{F}$ the cohomology of $M$ and $\operatorname{Fr}(\mathcal{F})$ a framing of $H^{0}\left(\left.\mathcal{F}\right|_{l_{\infty}}\right)$.

\section{Proof:}

Let $\mathcal{F}$ be a torsion free sheaf of rank 2 on $\mathbb{P}_{2}$ with $\left(c_{1}, c_{2}\right)=(0, n)$ trivial at $l_{\infty}$. We apply theorem 4.0.7 to $\mathcal{F}(-1)$. Because of lemma 4.0.6 there are only 3 non vanishing entries:

$$
0 \rightarrow H^{1}(\mathcal{F}(-2)) \otimes \mathcal{O}(-2) \rightarrow H^{1}(\mathcal{F} \otimes \Omega) \otimes \mathcal{O}(-1) \rightarrow H^{1}(\mathcal{F}(-1)) \otimes \mathcal{O} \rightarrow 0
$$

Thus $G_{p, q}^{2}=G_{p, q}^{\infty}$, therefore 4.0 .5 is a monad and $\mathcal{F}(-1)$ is its cohomology.

We tensor 4.0.5 with $\mathcal{O}_{1}$, denote the first arrow by $a$, the second by $b$ and set $H^{1}(\mathcal{F}(-2))=V, H^{1}(\mathcal{F} \otimes \Omega)=W$ and $H^{1}(\mathcal{F}(-1))=\tilde{V}$, then the monad becomes

$$
0 \rightarrow V \otimes \mathcal{O}(-1) \stackrel{a}{\rightarrow} W \otimes \mathcal{O} \stackrel{b}{\rightarrow} \tilde{V} \otimes \mathcal{O}(1) \rightarrow 0
$$

Because of lemma 4.0.6 one has $\chi(\mathcal{F}(-i))=-h^{1}(\mathcal{F}(-i))$ for $i=1,2$. Furthermore one has $\left(c_{1}(\mathcal{F}(-i))\left(, c_{2}(\mathcal{F}(-i))\right)=\left(-2 i, i^{2}+n\right)\right.$, which gives with 2.6.2 $-h^{1}(\mathcal{F}(-i))=\chi(\mathcal{F}(-i))=-n$ This shows $\operatorname{dim}(V)=\operatorname{dim}(\tilde{V})=n$. The same kind of computation shows $\operatorname{dim}(W)=2 n+2$. On the other hand a monad of the form 4.0.6 is uniquely defined by its cohomology (see [OSS]). Therefore the only thing left to be shown is that the cohomology $\mathcal{F}$ of any monad of the form 4.0.6 is torsion free with $\left(c_{1}(\mathcal{F}), c_{2}(\mathcal{F})\right)=(0, n)$. This means $\operatorname{ch}(\mathcal{F})=2-n h^{2}$. That can be verified by computing $\operatorname{ch}(k e r(b))=(n+2)-n h-\frac{n}{2} h^{2}$ from

$$
0 \rightarrow \operatorname{ker}(b) \rightarrow W \otimes \mathcal{O} \rightarrow \tilde{V} \otimes \mathcal{O}(1) \rightarrow 0
$$

and then computing $\operatorname{ch}(\mathcal{F})$ out of

$$
0 \rightarrow V \otimes \mathcal{O}(-1) \rightarrow \operatorname{ker}(b) \rightarrow \mathcal{F} \rightarrow 0
$$


And $\mathcal{F}$ is also torsion free: With 4.0.7 one shows, that $\underline{\operatorname{Ext}}{ }^{j}(k e r(b), \mathcal{O})=0$ for $j>0$, therefore $\operatorname{ker}(b)$ is locally free. With 4.0 .8 one gets $\underline{\operatorname{Ext}} t^{2}(\mathcal{F}, \mathcal{O})=0$. Furthermore since $\mathcal{F}$ is trivial at $l_{\infty}$ one has $\operatorname{setsupp}\left(\underline{\operatorname{Ext}} t^{1}(\mathcal{F}, \mathcal{O})\right) \cap l_{\infty}=\emptyset$, thus $\operatorname{setsupp}\left(\underline{\operatorname{Ext}} t^{1}(\mathcal{F}, \mathcal{O})\right)$ is of codimension 2, and therefore $\mathcal{F}$ torsion free due to lemma 2.4.2.

This completes the proof.

The final step of the proof of theorem 4.0.5

Lemma 4.0.9 Let $V$ and $\tilde{V}$ be vector spaces of dimension $n$ and $W$ a vector space of dimension $2 n+2$. There is a bijection from the set pairs $(M, F r(\mathcal{F}))$, where $M$ is a monad of the form

$$
0 \rightarrow V \otimes \mathcal{O}(-1) \stackrel{a}{\rightarrow} W \otimes \mathcal{O} \stackrel{b}{\rightarrow} \tilde{V} \otimes \mathcal{O}(1) \rightarrow 0
$$

$\mathcal{F}$ the cohomology of $M$ and $\operatorname{Fr}(\mathcal{F})$ a framing of $H^{0}\left(\left.\mathcal{F}\right|_{l_{\infty}}\right)$, to

$$
\left\{\begin{array}{cl}
(A)[A, B]+i j=0 \\
(\text { ii })(A, B, i, j) \text { satisfies }(\mathbf{S 1})
\end{array}\right\} / G L(n)
$$

\section{Proof:}

We choose homogenous coordinates $z_{0}, z_{1}, z_{2}$ of $\mathbb{P}_{2}$ such that $l_{\infty}=\left\{z_{0}=0\right\}$. One has $a \in H o m(V \otimes \mathcal{O}(-1), W \otimes \mathcal{O})=H^{0}(\mathcal{O}(1)) \otimes V^{*} \otimes W$. Thus choosing $z_{i}$ as basis of $H^{0}(\mathcal{O}(1))$ we write $a=\sum_{i=0}^{2} z_{i} a_{i}$, where $a_{i} \in \operatorname{Hom}(V, W)$. Furthermore $b=\sum_{i=0}^{2} z_{i} b_{i}$, where $b_{i} \in \operatorname{Hom}(W, V)$. We show, that $b_{2} a_{1}$ is an isomorphism: 4.0.8 tensored with $\mathcal{O}_{l_{\infty}}$ is still short exact, since $\mathcal{F}$ is trivial on $l_{\infty}$ :

$$
\left.\left.0 \rightarrow V \otimes \mathcal{O}_{l_{\infty}}(-1) \rightarrow \operatorname{ker}(b)\right|_{l_{\infty}} \rightarrow \mathcal{F}\right|_{l_{\infty}} \rightarrow 0
$$

By applying $H^{0}$ we get

$$
\tilde{W}:=H^{0}\left(\left.k e r(b)\right|_{l_{\infty}}\right) \cong H^{0}\left(\left.\mathcal{F}\right|_{l_{\infty}}\right)
$$

We choose a basis of $\tilde{W}$ to set the framing. Now let $s \in \tilde{W}$ be a nonvanishing section. Since 4.0.7 induces $\tilde{W} \hookrightarrow W$ we get $s(p)=w \in W \forall p \in l_{\infty}, \tilde{s} \in$ $H^{0}\left(\left.\mathcal{F}\right|_{l_{\infty}}\right)$ corresponding to $s$ under the isomorphism of 4.0.12. Then $\tilde{s}$ has a zero at $(0: 1: 0)$ if and only if there is a $v \in V$ such that 


$$
a_{1}(v)=w
$$

But this is equivalent to $\operatorname{im}\left(a_{1}\right) \cap \tilde{W} \neq \emptyset$ and since $\tilde{W}=\operatorname{ker}\left(b_{1}\right) \cap \operatorname{ker}\left(b_{2}\right)$ that means $\operatorname{im}\left(a_{1}\right) \cap \operatorname{ker}\left(b_{2}\right) \neq \emptyset$. Now $\left.\mathcal{F}\right|_{l_{\infty}}$ is trivial, therefore no nonvanishing section has a zero, thus we have

$$
\operatorname{im}\left(a_{1}\right) \cap \operatorname{ker}\left(b_{2}\right)=\emptyset .
$$

Tensoring 4.0.11 with $\mathcal{O}_{(0: 1: 0)}$ and $\mathcal{O}_{(0: 0: 1)}$ shows that $a_{1}, a_{2}$ are injective. Furthermore $b_{1}, b_{2}$ are surjective, therefore 4.0.13 gives $b_{2} a_{1}$ is an isomorphism. We identify $V$ and $\tilde{V}$ via this map.

The monad condition becomes with $a=\sum_{i=0}^{2} z_{i} a_{i}$ and $b=\sum_{i=0}^{2} z_{i} b_{i}$

$$
\begin{aligned}
& \text { (i) } b_{0} a_{0}=0 \quad \text { (ii) } b_{0} a_{1}+b_{1} a_{0}=0 \\
& \text { (iii) } b_{1} a_{1}=0 \quad \text { (iv) } b_{1} a_{2}+b_{2} a_{1}=0 \\
& \text { (v) } b_{2} a_{2}=0 \quad \text { (vi) } b_{0} a_{2}+b_{2} a_{0}=0
\end{aligned}
$$

These equations lead us to 4.0.10: We found $i m\left(a_{1}\right) \cap \tilde{W}=\emptyset$. The same argument shows $i m\left(a_{2}\right) \cap \tilde{W}=\emptyset$. And since $i m\left(a_{i}\right) \subset \operatorname{ker}\left(b_{i}\right)$ we can decompose $W=$ $i m\left(a_{1}\right) \oplus i m\left(a_{2}\right) \oplus \tilde{W}$. Identifying $i m\left(a_{i}\right)$ with $V$ via $a_{i}$ we get

$$
W=V \oplus V \oplus \tilde{W}
$$

Thus we have

$$
a_{1}=(\mathbf{1}, 0,0)^{t} \quad a_{2}=(0, \mathbf{1}, 0)^{t}
$$

and with $(i i i),(v), b_{2} a_{1}=\mathbf{1}$ and $(i v)$

$$
b_{1}=(0, \mathbf{1}, 0) \quad b_{2}=(-\mathbf{1}, 0,0)
$$

Now we set

$$
a_{0}=(B, A,-j)^{t} \quad b_{0}=(C, D, i)
$$

The equations (ii) and (vi) give us $C=-A$ and $B=D$. Thus $(i)$ becomes

$$
[A, B]+i j=0
$$


Now lemma 2.7 of $[N]$ says that having a tuple $(A, B, i, j)$ and related maps $a, b$ as above, then $\operatorname{ker}(a)=0$ and $b$ is surjective if and only if $\nexists S \subset V$ s.t $A(S) \subset S$, $B(S) \subset S$ and $i m(i) \subset S$. Thus a tuple $(A, B, i, j)$ gives rise to a monad if and only if it satisfies (S1). Furthermore we see that two tuples giving the same monad if and only if they belong to the same $G L(n)$-orbit.

This completes the proof.

Now we show that under the identification of theorem 4.0.5 $\mathcal{M}^{\text {reg }}(n)$ corresponds to the locally free sheaves in $\mathfrak{M}(n)$.

Lemma 4.0.10 Let $E \in \mathfrak{M}(n)$ with corresponding tuple $(A, B, i, j)$, then $E$ is locally free if and only if $(A, B, i, j)$ satisfies $(\mathbf{S 2})$.

\section{Proof:}

Consider

$$
0 \rightarrow V \otimes \mathcal{O}(-1) \stackrel{a}{\rightarrow} \operatorname{ker}(b) \rightarrow E \rightarrow 0
$$

By lemma 2.5.1 $E$ is not locally free if and only if there is a point $z \in \mathbb{P}_{2}$ such that $\operatorname{Tor}^{1}\left(E, \mathcal{O}_{z}\right) \neq 0$. Since $\operatorname{ker}(b)$ is locally free we see by tensoring 4.0.14 with $\mathcal{O}_{z}$ that $\operatorname{Tor}^{1}\left(E, \mathcal{O}_{z}\right) \neq 0$ is equivalent to the existence of a point $z$ such that $a_{z}$ is not injective. But that means $\hat{b}$ the map resulting from $\left(B^{t}, A^{t}, j^{t}, i^{t}\right)$ is not surjective and by lemma 2.7 of $[\mathrm{N}]$ that means, that $\left(B^{t}, A^{t}, j^{t}, i^{t}\right)$ does not satisfy condition $(\mathbf{S 1})$, which is equivalent to condition $(\mathbf{S 2})$ for $(A, B, i, j)$.

This completes the proof. 


\section{Chapter 5}

\section{The $\mathfrak{G}$-action on $\mathcal{M}_{c}^{r e g}(n)$ for $n \leq 4$}

From now on we will consider the unframed versions of $\mathcal{M}^{\text {reg }}(n)$ and $\mathfrak{M}(n)$. We set

$$
\tilde{\mathcal{M}}^{r e g}(n):=\mathcal{M}^{r e g}(n) / G L(2), \quad \tilde{\mathfrak{M}}(n)=\mathfrak{M}(n) / G L(2)
$$

Note that the $\mathfrak{G}$-action descents to $\tilde{\mathcal{M}}^{\text {reg }}(n)$.

\section{1 $\tilde{\mathcal{M}}_{c}^{r e g}(n)$ and $\tilde{\mathcal{M}}_{n c}^{r e g}(n)$}

We will show, that there is no element of $\tilde{\mathcal{M}}^{\text {reg }}(n)$ such that for the corresponding $(A, B, i, j)$ one has $r k[A, B]=1$. Therefore we can decompose

$$
\tilde{\mathcal{M}}^{\text {reg }}(n)=\tilde{\mathcal{M}}_{c}^{\text {reg }}(n) \sqcup \tilde{\mathcal{M}}_{n c}^{\text {reg }}(n),
$$

where $\tilde{\mathcal{M}}_{c}^{\text {reg }}(n)$ denotes the set of instantons such that for the corresponding $(A, B, i, j)$ we have $[A, B]=0$, and $\tilde{\mathcal{M}}_{n c}^{r e g}(n)$ denotes the set of instantons such that for the corresponding $(A, B, i, j)$ we have $r k[A, B]=2$.

Furthermore we show, that an element of $\tilde{\mathcal{M}}_{n c}^{\text {reg }}(n)$ is already uniquely defined by the corresponding $(A, B)$.

Lemma 5.1.1 There is no element of $\tilde{\mathcal{M}}^{\text {reg }}(n)$ such that for the corresponding $(A, B, i, j)$ one has $r k[A, B]=1$.

\section{Proof:}

Due to lemma 4.0.10 an element of $\tilde{\mathcal{M}}^{\text {reg }}(n)$ corresponds to a locally free sheaf $E$, thus the conditions of [OSS] p.279 Corollary 2 are satisfied. Therefore the adjoint of the monad of $E$ is also the monad of $E$, since $E=E^{*}$. This implies $r k(i)=r k(j)$. 
So if $r k[A, B]=1$, one must have $r k(j)=r k(i)=1$. Now we choose a basis of $V$ such that $[A, B]$ has Jordan normal form. Since it must be trace free one has

$$
[A, B]=\left(\begin{array}{ccccc}
0 & 1 & 0 & \cdots & 0 \\
0 & 0 & 0 & & 0 \\
\vdots & \vdots & \vdots & & \vdots \\
& & & & \\
0 & 0 & 0 & \cdots & 0
\end{array}\right)
$$

and taking the suitable basis of $\tilde{W}$

$$
i=\left(\begin{array}{cc}
1 & 0 \\
0 & 0 \\
\vdots & \vdots \\
0 & 0
\end{array}\right), \quad j=\left(\begin{array}{ccccc}
0 & 1 & 0 & \cdots & 0 \\
0 & 0 & 0 & \cdots & 0
\end{array}\right)
$$

Now let $C$ be any word in $A$ and $B$. We show by induction on the length of $C$, that $j C i=0$. But that means, since $j \neq 0$ that the subspace $S=$ $\operatorname{span}\{C i(\tilde{W}) \mid C$ is a word in $A, B\}$ contradict condition (S2).

If $C$ is of length 0 one computes $j i=0$. Now let $C$ be of length $k$. Then one has

$$
j C i=\left(\begin{array}{cc}
c_{21} & 0 \\
0 & 0
\end{array}\right), \quad C i j=\left(\begin{array}{ccccc}
0 & c_{11} & 0 & \cdots & 0 \\
0 & c_{21} & 0 & & 0 \\
\vdots & \vdots & \vdots & & \vdots \\
& & & & \\
0 & c_{n 1} & 0 & \cdots & 0
\end{array}\right)
$$

So we have to show, that $\operatorname{tr}(j C i)=\operatorname{tr}(C i j)=0$. The proof of that is taken from [N] p.24: We denote for the moment $A, B$ by $D_{1}, D_{2}$. So we can write $C=D_{\alpha_{1}} \cdots D_{\alpha_{k}}$ where $\alpha_{l}=1$ or $=2$. If there is a $l$ such that $D_{\alpha_{l-1}}=B$, $D_{\alpha_{l}}=A$, we have

$$
\begin{aligned}
j C & =j D_{\alpha_{1}} \cdots B A \cdots D_{\alpha_{k}} \\
& =j D_{\alpha_{1}} \cdots([B, A]+A B) \cdots D_{\alpha_{k}} \\
& =\left(j D_{\alpha_{1}} \cdots i\right) j \cdots D_{\alpha_{k}}+j D_{\alpha_{1}} \cdots A B \cdots D_{\alpha_{k}} \\
& =j D_{\alpha_{1}} \cdots A B \cdots D_{\alpha_{k}}
\end{aligned}
$$

where the last equality results from the induction hypothesis. So it suffices to proof $\operatorname{tr}(i C j)=0$ for $C=A^{k-l} B^{l}$ : 


$$
\begin{aligned}
\operatorname{tr}(j C i) & =\operatorname{tr}(C i j)=-\operatorname{tr}\left(A^{k-l} B^{l}[A, B]\right) \\
& \left.=-\operatorname{tr}\left(\left[A^{k-l} B^{l}, A\right] B\right]\right)=-\operatorname{tr}\left(A^{k-l}\left[B^{l}, A\right] B\right) \\
& =-\sum_{p=0}^{p=l-1} \operatorname{tr}\left(A^{k-l} B^{p}[B, A] B^{l-p-1} B\right) \\
& =-\sum_{p=l-1}^{p=0} \operatorname{tr}\left(B^{l-p} A^{k-l} B^{p}[B, A]\right) \\
& =-\sum_{p=0}^{p=l-1} \operatorname{tr}\left(B^{l-p} A^{k-l} B^{p} i j\right) \\
& =-\sum_{p=0}^{p=l-1} \operatorname{tr}\left(j B^{l-p} A^{k-l} B^{p} i\right)
\end{aligned}
$$

Thus with 5.1.1 we get

$$
\operatorname{tr}(j C i)=-l \cdot \operatorname{tr}(j C i)
$$

This completes the proof.

A commutator has a vanishing trace, therefore for $n=2$ there is up to a factor only one possible conjugation class of $C=[A, B]$. For $n=3$ there is also the possibility that $C$ is nilpotent and for $n \geq 4$ a nilpotent $C$ can be either satisfy $C^{2}=0$ or $C^{2} \neq 0$. Therefore for $n \geq 4$ up to a factor there are 3 possible conjugation classes of $C$. We give 3 examples for $n=4$ to show that all possibilities occur.

Let $A$ always be

$$
A=\left(\begin{array}{llll}
0 & 1 & 0 & 0 \\
0 & 0 & 1 & 0 \\
0 & 0 & 0 & 1 \\
0 & 0 & 0 & 0
\end{array}\right)
$$

- with

$$
B=\left(\begin{array}{llll}
0 & 0 & 0 & 0 \\
1 & 0 & 0 & 0 \\
0 & 1 & 0 & 0 \\
0 & 0 & 1 & 0
\end{array}\right)
$$

Then

$$
C=\left(\begin{array}{cccc}
1 & 0 & 0 & 0 \\
0 & 0 & 0 & 0 \\
0 & 0 & 0 & 0 \\
0 & 0 & 0 & -1
\end{array}\right) \quad i=\left(\begin{array}{cc}
1 & 0 \\
0 & 0 \\
0 & 0 \\
0 & 1
\end{array}\right) \quad j=\left(\begin{array}{cccc}
1 & 0 & 0 & 0 \\
0 & 0 & 0 & -1
\end{array}\right)
$$


This tuple is stable: One has

$$
A(i m(i))=\mathbb{C}\left(\begin{array}{l}
0 \\
0 \\
1 \\
0
\end{array}\right) \quad B(i m(i))=\mathbb{C}\left(\begin{array}{l}
0 \\
1 \\
0 \\
0
\end{array}\right)
$$

and since $\operatorname{span}\{i m(i), A(i m(i)), B(i m(i))\}$ is of dimension $4(\mathbf{S 1})$ is satisfied. The same is true for the transposed tuple, thus (S2) is also satisfied, and $C$ is not nilpotent.

- with

$$
B=\left(\begin{array}{llll}
0 & 0 & 0 & 0 \\
0 & 0 & 0 & 0 \\
0 & 0 & 0 & 0 \\
1 & 0 & 0 & 0
\end{array}\right)
$$

Then

$$
C=\left(\begin{array}{cccc}
0 & 0 & 0 & 0 \\
0 & 0 & 0 & 0 \\
1 & 0 & 0 & 0 \\
0 & -1 & 0 & 0
\end{array}\right) \quad i=\left(\begin{array}{ll}
0 & 0 \\
0 & 0 \\
1 & 0 \\
0 & 1
\end{array}\right) \quad j=\left(\begin{array}{cccc}
1 & 0 & 0 & 0 \\
0 & -1 & 0 & 0
\end{array}\right)
$$

This tuple is stable since $\operatorname{dim}\left(\operatorname{span}\left\{i m(i), A^{2}(i m(i))\right\}\right)=4$ and $\operatorname{dim}\left(\operatorname{span}\left\{i m\left(j^{t}\right), A^{2}\left(i m\left(j^{t}\right)\right)\right\}\right)=4$. One has $C^{2}=0$.

- with

$$
B=\left(\begin{array}{llll}
0 & 0 & 0 & 0 \\
0 & 0 & 0 & 0 \\
1 & 0 & 0 & 0 \\
1 & 0 & 0 & 0
\end{array}\right)
$$

Then 


$$
C=\left(\begin{array}{cccc}
0 & 0 & 0 & 0 \\
1 & 0 & 0 & 0 \\
1 & -1 & 0 & 0 \\
0 & -1 & 0 & 0
\end{array}\right) \quad i=\left(\begin{array}{cc}
0 & 0 \\
1 & 0 \\
1 & -1 \\
0 & -1
\end{array}\right) \quad j=\left(\begin{array}{cccc}
1 & 0 & 0 & 0 \\
0 & 1 & 0 & 0
\end{array}\right)
$$

This tuple is stable: One has

$$
A^{2}(i m(i))=\operatorname{span}\left\{\left(\begin{array}{l}
1 \\
0 \\
0 \\
0
\end{array}\right),\left(\begin{array}{c}
-1 \\
-1 \\
0 \\
0
\end{array}\right)\right\}
$$

thus $\operatorname{span}\left\{\mathrm{im}(i), A^{2}(\mathrm{im}(i))\right\}$ is of dimension 4 and $(\mathbf{S 1})$ is satisfied. As in the previous case also the transposed tuple satisfies $(\mathbf{S 1})$. One has $C^{2} \neq 0$ but $C^{3}=0$.

Lemma 5.1.2 Let $A, B \in \operatorname{gl}(V)$ s.t. $\quad r k[A, B]=2$. If $A, B$ is part of two $A D H M$ data $(A, B, i, j)$ and $\left(A, B, i^{\prime}, j^{\prime}\right)$, then there is an $s \in G L(2)$ such that $(\mathbf{1}, s)(A, B, i, j)=(A, B, \tilde{i}, \tilde{j})$.

\section{Proof:}

Consider 2 data $(A, B, i, j)$ and $(A, B, \tilde{i}, \tilde{j})$. Let $C=[A, B]$ we show the claim for $C$ having Jordan normal form

$$
C=\left(\begin{array}{ccccc}
\eta & 0 & 0 & \cdots & 0 \\
0 & -\eta & 0 & \cdots & 0 \\
0 & 0 & 0 & \cdots & 0 \\
\vdots & \vdots & \vdots & \ddots & \\
0 & 0 & 0 & \cdots & 0
\end{array}\right)
$$

for the other at most 2 Jordan normal forms one proves the claim in the same way. In a basis where $C$ has Jordan normal form we have

$$
i=\left(\begin{array}{cc}
i_{11} & i_{12} \\
i_{21} & i_{22} \\
0 & 0 \\
\vdots & \vdots \\
0 & 0
\end{array}\right) \quad j=\left(\begin{array}{ccccc}
j_{11} & j_{12} & 0 & \cdots & 0 \\
j_{21} & j_{22} & 0 & \cdots & 0
\end{array}\right)
$$


and $\tilde{i}, \tilde{j}$ are of the same form. Let

$$
\check{j}=\left(\begin{array}{cc}
\tilde{j}_{11} & \tilde{j}_{12} \\
\tilde{j}_{21} & \tilde{j}_{22}
\end{array}\right) \text { and set } \hat{j}=\left(\begin{array}{c}
\check{j}^{-1} \\
0 \\
\vdots \\
0
\end{array}\right)
$$

Then with $s:=j \hat{j}$ we have $(\mathbf{1}, s)(A, B, i, j)=(A, B, \tilde{i}, \tilde{j})$, since $\tilde{j} \hat{j}=\mathbf{1}$.

This completes the proof.

\subsection{Stable pairs}

We show that $(A, B, i, j) \in \tilde{\mathcal{M}}_{c}^{r e g}(n)$ is not uniquely determined by the pair $(A, B)$. We therefore introduce the set of stable pairs $\mathcal{S}(n)$ and compute the number of $\mathfrak{G}$-orbits in $\mathcal{S}(n)$ for $n \leq 4$.

Lemma 5.2.1 If $(A, B, i, j)$ represents an element of $\tilde{\mathcal{M}}_{c}^{r e g}(n)$, then $r k(i)=$ $r k(j)=1$

\section{Proof:}

Since we know, that $r k(i)=r k(j)$, see lemma 4.0.10, there are only two possibilities. If $r k(i)=r k(j)=0$, then the span of the common eigenvector of $A$ and $B$ violates (S2).

This completes the proof.

Definition 5.2.2 Let $(A, B) \in \operatorname{gl}(n) \times g l(n)$. Then $(A, B)$ is called stable if it satisfied the following two conditions:

(s1) There is no common eigenspace of $A^{t}, B^{t}$ of dimension $\geq 2$.

(s2) There is no common eigenspace of $A, B$ of dimension $\geq 2$.

We denote the set of conjugation classes of stable pairs of size $n$ by $\mathcal{S}(n)$.

Lemma 5.2.3 For a given pair of matrices $(A, B)$ with $[A, B]=0$ there exists $i, j$ such that the $A D H M$ datum $(A, B, i, j)$ is stable if and only if $(A, B)$ is stable. In this case the $G L(2)$-orbit of $i, j$ is not uniquely defined by $(A, B)$. 


\section{Proof:}

Let $(A, B)$ such that there are $i, j$ such that $(A, B, i, j)$ is stable. Suppose $(A, B)$ does not satisfy (s2), then since due to lemma 5.2.1 $\operatorname{dim}(\operatorname{ker}(j))=n-1, S \cap$ $\operatorname{ker}(j)=: \tilde{S} \neq \emptyset$, and $\tilde{S}$ violates (S2) for $(A, B, i, j)$, which is a contradiction. Now let $(A, B)$ satisfy (s2), then the number of invariant subspaces of $(A, B)$ is finite. Therefore we can choose $j$ such that non of these invariant subspaces is a subspace of $\operatorname{ker}(j)$ and this choice is not unique.

(s1) is (s2) for $\left(B^{t}, A^{t}\right)$. Thus (s1) is equivalent to (S2) for $\left(B^{t}, A^{t}, j^{t}, i^{t}\right)$, which is equivalent to $(\mathbf{S} 1)$ for $(A, B, i, j)$.

This completes the proof.

Remark: Because of lemma 5.2.3 for a given stable pair $(A, B)$ there are families of pairs $(i, j)$ such that $(A, B, i, j)$ represents an instanton. Furthermore for a generic choice of $(i, j)$ and $\left(i^{\prime}, j^{\prime}\right),(A, B, i, j)$ and $\left(A, B, i^{\prime}, j^{\prime}\right)$ do not belong to the same $\mathcal{G}$-orbit. Therefore we will investigate the $\mathcal{G}$ action on stable pairs.

Lemma 5.2.4 There is a dense $\mathfrak{G}$-orbit in $\mathcal{S}(n)$. It consists of all diagonalizable stable pairs.

\section{Proof:}

Let $(A, B)=\left(\operatorname{diag}\left(a_{1}, \cdots, a_{n}\right), \operatorname{diag}\left(b_{1}, \cdots b_{n}\right)\right)$. We show that we can map it to $(\tilde{A}, \tilde{B})=(\operatorname{diag}(1,2, \cdots n), \operatorname{diag}(-1,-2, \cdots,-n))$. Since $(A, B)$ is stable there is a $\lambda \in \mathbb{C}$ such that $A+\lambda B=\operatorname{diag}\left(\tilde{a}_{1}, \ldots ., \tilde{a}_{n}\right)$ has no two dimensional eigenspace. Let

$$
p(x):=\sum_{k=1}^{n} p_{k}(x)=\sum_{k=1}^{n}\left(-k-b_{k}\right) \prod_{i=1 ; i \neq k}^{n} \frac{x-\tilde{a}_{i}}{\tilde{a}_{k}-\tilde{a}_{i}}
$$

and $a=(x, y) \mapsto(A+\lambda B, B)$. Then $e_{p}(a(A, B))=(A+\lambda B, \tilde{B})$. Now we map $(A+\lambda B, \tilde{B})$ to $(\tilde{B}, A+\lambda B)$ which is mapped by $e_{\tilde{p}}$ to $(\tilde{B}, \tilde{A})$ where

$$
\tilde{p}(x):=\sum_{k=1}^{n} p_{k}(x)=\sum_{k=1}^{n}\left(k-\tilde{a}_{k}\right) \prod_{i=1 ; i \neq k}^{n} \frac{x+i}{i-k}
$$

Since the $\mathfrak{G}$-action preserves the condition of being diagonalizable there are no further pairs in this orbit

This completes the proof. 


\section{3 $\tilde{\mathcal{M}}_{c}^{r e g}(2)$ and $\tilde{\mathcal{M}}_{c}^{r e g}(3)$}

Definition 5.3.1 Let $(A, B) \in g l(n) \times g l(n)$ and consider $\mathbb{P}_{2}$ as the scheme $\operatorname{Proj}\left(\mathbb{C}\left[\lambda_{0}, \lambda_{1}, \lambda_{2}\right]\right)$. The subscheme $\Sigma_{(A, B)} \subset \mathbb{P}_{2}$ given by homogeneous ideal generated by

$$
\operatorname{det}\left(\lambda_{0} A+\lambda_{1} B-\lambda_{2} \mathbf{1}\right)
$$

is called the spectral scheme of $(A, B)$. We set $\operatorname{supp}\left(\Sigma_{(A, B)}\right)=: \tilde{\Sigma}_{(A, B)}$ and called it the spectral curve.

Since $\Sigma_{(A, B)}$ is invariant under conjugation of $(A, B)$ this is a well defined object for $(A, B)^{G L(n)}$, the $G L(n)$-orbit of $(A, B)$.

Lemma 5.3.2 There are two $\mathfrak{G}$-orbits in $\mathcal{S}(2)$. They are characterized by the spectral curves of their elements: One orbit consists of classes of stable pairs such that their spectral curves consist of two non identical lines (the dense orbit), the other one of classes of stable pairs with a line as spectral curve.

\section{Proof:}

If $A^{G L(2)}$ and $B^{G L(2)}$ of a class $(A, B)^{G L(2)}$ have only one eigenvalue each, then one of them must not be a multiply of the identity, say $A^{G L(N)}$, since otherwise the pair would not be stable. Thus $(A, B)^{G L(2)}$ has a representative

$$
A=\left(\begin{array}{cc}
a & \alpha \\
0 & a
\end{array}\right) \quad B=\left(\begin{array}{cc}
b & \beta \\
0 & b
\end{array}\right)
$$

with $\alpha \neq 0$, and we can assume $a \neq 0$. We perform the following mappings: $(x, y) \rightarrow\left(x, y+1-\frac{b}{a} x\right)$ and $(x, y) \mapsto\left(x, y+\left(1-\left(\frac{\beta}{\alpha}-\frac{b}{a}\right)(x-a)\right)\right.$ then we get

$$
\left(\left(\begin{array}{ll}
a & \alpha \\
0 & a
\end{array}\right),\left(\begin{array}{ll}
1 & 1 \\
0 & 1
\end{array}\right)\right)
$$

then we act with $(x, y) \mapsto(y, x)$ and use mappings like above to get

$$
\left(\left(\begin{array}{ll}
1 & 1 \\
0 & 1
\end{array}\right),\left(\begin{array}{ll}
1 & 1 \\
0 & 1
\end{array}\right)\right)
$$

This completes the proof.

Lemma 5.3.3 There are three $\mathfrak{G}$-orbits in $\mathcal{S}(3)$. They are characterized by the degree of the spectral curve. 


\section{Proof:}

If $A$ and $B$ have both one eigenvalue only, we have to consider the following cases:

1. One of the two classes $A^{G L(3)}, B^{G L(3)}$ say $A^{G L(3)}$ has only one Jordan block, then there is a representative of $(A, B)^{G L(3)}$ of the form

$$
A=\left(\begin{array}{ccc}
a & \alpha & \delta \\
0 & a & \alpha \\
0 & 0 & a
\end{array}\right), \quad B=\left(\begin{array}{ccc}
b & \beta & \gamma \\
0 & b & \beta \\
0 & 0 & b
\end{array}\right) .
$$

with $\alpha \neq 0$. All these classes belong to the same orbit: We perform the following mappings: $(x, y) \mapsto\left(x, y+\left(1-\frac{b}{q} x\right)\right) ;(x, y) \mapsto\left(x, y+\left(1-\left(\frac{\beta}{\alpha}-\right.\right.\right.$ $\left.\left.\frac{a}{b}\right)(x-a)\right)$ and $(x, y) \mapsto\left(x, y+\left(1-\left(\frac{\gamma}{2 \delta}-\frac{b}{a}\right)(x-a)^{2}\right)\right.$ to get

$$
\left(\begin{array}{lll}
a & \alpha & \delta \\
0 & a & \alpha \\
0 & 0 & a
\end{array}\right),\left(\begin{array}{lll}
1 & 1 & 1 \\
0 & 1 & 1 \\
0 & 0 & 1
\end{array}\right)
$$

After an interchange of the matrices we perform similar mappings to get

$$
\left(\begin{array}{lll}
1 & 1 & 1 \\
0 & 1 & 1 \\
0 & 0 & 1
\end{array}\right),\left(\begin{array}{lll}
1 & 1 & 1 \\
0 & 1 & 1 \\
0 & 0 & 1
\end{array}\right) .
$$

2. One of the two classes $A^{G L(3)}, B^{G L(3)}$ say $A^{G L(3)}$ has 2 Jordan blocks, then there is a representative

$$
A=\left(\begin{array}{ccc}
a & 0 & 0 \\
0 & a & 1 \\
0 & 0 & a
\end{array}\right) \quad B=\left(\begin{array}{ccc}
b & 0 & \gamma \\
\alpha & b & \beta \\
0 & 0 & b
\end{array}\right)
$$

of $(A, B)^{G L(3)}$. If $\alpha=0$ then with $S=\operatorname{span}\left\{e_{1}, e_{2}\right\}$ we violate condition (s2). If $\gamma=0$ we violate condition (s1) with $S=\operatorname{span}\left\{e_{1}, e_{3}\right\}$. The equation

$$
B(v)=b \cdot v+\left(\begin{array}{c}
\gamma v_{3} \\
\alpha v_{1}+\beta v_{3} \\
0
\end{array}\right)
$$

therefore shows that $B$ has only one eigenvector. Thus if we permute $A, B$ and bring $B$ in Jordan normal form, we are in situation 1). 
If one of the matrices $A, B$ has two different eigenvalues, we can assume that

$$
A=\left(\begin{array}{ccc}
a_{1} & 0 & 0 \\
0 & a_{2} & 1 \\
0 & 0 & a_{2}
\end{array}\right) \quad B=\left(\begin{array}{ccc}
b_{1} & 0 & 0 \\
0 & b_{2} & \beta \\
0 & 0 & b_{2}
\end{array}\right)
$$

with $a_{1} \neq a_{2}$. By multiplying the polynomials, that we used for charge 2 with $\left(x-a_{1}\right)$ and then acting with the appropriate multiply of $\left(x-a_{2}\right)^{2}$, interchanging the matrices and repeating the same process, we reach

$$
\left(\begin{array}{lll}
2 & 0 & 0 \\
0 & 1 & 1 \\
0 & 0 & 1
\end{array}\right),\left(\begin{array}{lll}
2 & 0 & 0 \\
0 & 1 & 1 \\
0 & 0 & 1
\end{array}\right) .
$$

The $\mathfrak{G}$-action preserves the common invariant subspaces of $A$ and $B$. Therefore these pairs form a second orbit. The third is the dense one.

This completes the proof.

\section{$\mathbf{5 . 4} \tilde{\mathcal{M}}_{c}^{r e g}(4)$}

In charge 4 the spectral curve does not suffice to characterize the existing $\mathfrak{G}$-orbits in $\mathcal{S}(4)$ :

Lemma 5.4.1 Let $\Delta \subset \mathcal{S}(4)$ be the set of classes of stable pairs that have a line as spectral curve. Then there are two $\mathfrak{G}$-orbits in $\Delta$.

\section{Proof:}

We have to consider the following cases:

1. One of the two classes $A^{G L(4)}, B^{G L(4)}$ say $A^{G L(4)}$, has only one Jordan block, then we act as we did in the case of charge 3: A representative of $(A, B)^{G L(4)}$ is

$$
A=\left(\begin{array}{cccc}
a & 1 & 0 & 0 \\
0 & a & 1 & 0 \\
0 & 0 & a & 1 \\
0 & 0 & 0 & a
\end{array}\right) \quad B=\left(\begin{array}{cccc}
b & \alpha & \beta & \gamma \\
0 & b & \alpha & \beta \\
0 & 0 & b & \alpha \\
0 & 0 & 0 & b
\end{array}\right)
$$

We can always map $(A, B)$ to 


$$
\left(\begin{array}{llll}
1 & 1 & 0 & 0 \\
0 & 1 & 1 & 0 \\
0 & 0 & 1 & 1 \\
0 & 0 & 0 & 1
\end{array}\right),\left(\begin{array}{llll}
1 & 1 & 0 & 0 \\
0 & 1 & 1 & 0 \\
0 & 0 & 1 & 1 \\
0 & 0 & 0 & 1
\end{array}\right)
$$

by acting with $(x, y) \mapsto(x+(1-a), y+(1-b))$ and $(x, y) \mapsto(x, y+(1-$ $\left.\alpha)(x-1)-\beta(x-1)^{2}-\gamma(x-1)^{3}\right)$. Thus all these classes of pairs belong to one orbit $\mathcal{O}_{1}$.

2. One of the two classes $A^{G L(4)}, B^{G L(4)}$ say $A^{G L(4)}$ has a Jordan block of size 1 and a Jordan block of size 3 , then there is a representative

$$
A=\left(\begin{array}{cccc}
a & 0 & 0 & 0 \\
0 & a & 1 & 0 \\
0 & 0 & a & 1 \\
0 & 0 & 0 & a
\end{array}\right) \quad B=\left(\begin{array}{cccc}
b & 0 & 0 & \eta \\
\tau & b & \beta & \delta \\
0 & 0 & b & \beta \\
0 & 0 & 0 & b
\end{array}\right)
$$

Stability implies $\tau \neq 0$ and $\eta \neq 0$. By using $(x, y) \mapsto(x, y-\beta(x-a \mathbf{1})-$ $\left.\delta(A-a \mathbf{1})^{2}\right)$ followed by a conjugation with $\operatorname{diag}(1, \tau, \tau, \tau)$, which is in the stabilizer of $A$, we get

$$
\tilde{B}=\left(\begin{array}{llll}
b & 0 & 0 & \xi \\
1 & b & 0 & 0 \\
0 & 0 & b & 0 \\
0 & 0 & 0 & b
\end{array}\right)
$$

with $\xi=\tau \eta \neq 0$. We will show after considering the last case, that these pairs belong to a second orbit $\mathcal{O}_{2}$.

3. One of the two classes $A^{G L(4)}, B^{G L(4)}$ say $A^{G L(4)}$ has two Jordan blocks of size 2. Since the choice of $B$ is unique only up to conjugation with $S t a b_{G L(4)} A$, we can choose a representative

$$
A=\left(\begin{array}{cccc}
a & 1 & 0 & 0 \\
0 & a & 0 & 0 \\
0 & 0 & a & 1 \\
0 & 0 & 0 & a
\end{array}\right) \quad B=\left(\begin{array}{cccc}
b & \alpha & \delta & \gamma \\
0 & b & 0 & \delta \\
0 & \eta & b & \beta \\
0 & 0 & 0 & b
\end{array}\right)
$$


Because $(A, B)$ has to be stable we need $\delta \neq 0$. The equation

$$
B(v)=b \cdot v+\left(\begin{array}{c}
\alpha v_{2}+\delta v_{3}+\gamma v_{4} \\
\delta v_{4} \\
\eta v_{2}+\beta v_{4} \\
0
\end{array}\right)
$$

shows that $B$ has a 2 dimensional eigenspace if and only if $\eta=0$. Therefore $(A, B)^{G L(4)} \in \mathcal{O}_{1}$ if $\eta \neq 0$. So let $\eta=0$. A, B have a two dimensional eigenspace each, and the intersection of these eigenspaces is of dimension one. These eigenspace conditions are preserved by the $\mathcal{G}$-action. Therefore $(A, B)^{G L(4)}$ cannot belong to $\mathcal{O}_{1}$. So we have found that there is at least one other orbit. We show, that all pairs of this form belong to one orbit:

A conjugation with

$$
g=\left(\begin{array}{cccc}
1 & \frac{\gamma}{\delta} & 0 & 0 \\
0 & 1 & 0 & 0 \\
0 & 0 & \delta^{-1} & 0 \\
0 & 0 & 0 & \delta^{-1}
\end{array}\right)
$$

which is in $\operatorname{Stab}_{G L(4)} A$ and an action of $(x, y) \mapsto(x, y-\alpha(x-a))$ maps $B$ to

$$
\tilde{B}=\left(\begin{array}{llll}
b & 0 & 1 & 0 \\
0 & b & 0 & 1 \\
0 & 0 & b & \xi \\
0 & 0 & 0 & b
\end{array}\right)
$$

If $\xi=0$ which means $\alpha=\beta$, then $A$ and $B$ have both two Jordan blocks of size 2 . By acting with $(x, y) \mapsto(x-a, y-b)$ we can map any such pair to the class having the representative

$$
\left(\begin{array}{llll}
0 & 1 & 0 & 0 \\
0 & 0 & 0 & 0 \\
0 & 0 & 0 & 1 \\
0 & 0 & 0 & 0
\end{array}\right),\left(\begin{array}{llll}
0 & 0 & 1 & 0 \\
0 & 0 & 0 & 1 \\
0 & 0 & 0 & 0 \\
0 & 0 & 0 & 0
\end{array}\right) .
$$


Thus they belong to one orbit $\mathcal{O}_{2}$. If $\xi \neq 0$ then $\tilde{B}$ has a Jordan block of size 3 , since $(\tilde{B}-b \mathbf{1})^{2} \neq 0$. Therefore after permuting $A$ and $\tilde{B}$ we have mapped $(A, B)^{G L(4)}$ to a class of 2$)$.

Now we show, that we can map

$$
A=\left(\begin{array}{llll}
a & 1 & 0 & 0 \\
0 & a & 0 & 0 \\
0 & 0 & a & 1 \\
0 & 0 & 0 & a
\end{array}\right) \quad B=\left(\begin{array}{cccc}
b & 0 & 1 & 0 \\
0 & b & 0 & 1 \\
0 & 0 & b & 0 \\
0 & 0 & 0 & b
\end{array}\right)
$$

to

$$
\left(\begin{array}{llll}
a & 0 & 0 & 0 \\
0 & a & 1 & 0 \\
0 & 0 & a & 1 \\
0 & 0 & 0 & a
\end{array}\right),\left(\begin{array}{llll}
b & 0 & 0 & \xi \\
1 & b & 0 & 0 \\
0 & 0 & b & 0 \\
0 & 0 & 0 & b
\end{array}\right)
$$

For any given $\xi \neq 0$. That shows, that all classes of 2. and 3 . belong to $\mathcal{O}_{2}$. At first we map $(A, B)$ to $\left(C_{\lambda}, D\right):=(A-\lambda B, A+B)$. For the moment we only assume $\lambda \neq 0$. Let

$$
M:=\left(\begin{array}{cccc}
0 & 1 & 0 & 0 \\
\lambda & 0 & \frac{1}{2} & \lambda \\
1 & 0 & -\frac{1}{2 \lambda} & 1 \\
0 & 0 & 0 & -\frac{1}{2 \lambda}
\end{array}\right)
$$

then we have with $c_{\lambda}=a-\lambda b$ and $d=a+b$

$$
C_{\lambda}^{M}=\left(\begin{array}{cccc}
c_{\lambda} & 0 & 0 & 0 \\
0 & c_{\lambda} & 1 & 0 \\
0 & 0 & c_{\lambda} & 1 \\
0 & 0 & 0 & c_{\lambda}
\end{array}\right) ; \quad D^{M}=\left(\begin{array}{cccc}
d & 0 & 0 & -\frac{1+\lambda}{4 \lambda^{2}} \\
1+\lambda & d & \frac{\lambda-1}{2 \lambda} & 1+\lambda \\
0 & 0 & d & \frac{\lambda-1}{2 \lambda} \\
0 & 0 & 0 & d
\end{array}\right)
$$

Thus $\left(C_{\lambda}^{M}, D^{M}\right)$ is of the form 2. Using the same maps as there we can map $\left(C_{\lambda}^{M}, D^{M}\right)$ to

$$
\left(\left(\begin{array}{cccc}
a & 0 & 0 & 0 \\
0 & a & 1 & 0 \\
0 & 0 & a & 1 \\
0 & 0 & 0 & a
\end{array}\right),\left(\begin{array}{cccc}
b & 0 & 0 & -\frac{1}{4 \lambda^{2}} \\
1 & b & 0 & 0 \\
0 & 0 & b & 0 \\
0 & 0 & 0 & b
\end{array}\right)\right)
$$


Thus for a given $\xi$ we have to choose $\lambda=\frac{i}{2} \xi^{-\frac{1}{2}}$.

This completes the proof.

As explicitly done in the proof of charge 3 one can "manipulate" the matrices $A$ and $B$ in each common generalized eigenspace separately. Therefore we get

Corollary 5.4.2 There are $6 \mathfrak{G}$-orbits in $\mathcal{S}(4)$ : One s.t. a pair belonging to it has 4 lines, one s.t. a pair belonging to it has 3 lines, two s.t. a pair belonging to it has 2 lines and two s.t. a pair belonging to it has 1 line as spectral curve. 


\section{Chapter 6}

\section{The $\mathfrak{G}$-action on $\mathcal{M}_{n c}^{r e g}(2)$ and $\mathcal{M}_{n c}^{\text {reg }}(3)$}

\subsection{A dense orbit in $\mathcal{M}_{n c}^{r e g}(n)$}

The adjoint action of $G L(n)$ on $g l(n) \times g l(n)$ is a symplectic action with moment map $m: g l(n) \times g l(n) \rightarrow g l(n) ; m((A, B))=[A, B]$.

In $[\mathrm{A} 1]$ Artamkin shows the following lemma:

Lemma 6.1.1 Let $0 \neq C \in g l(n)$. Then there is a dense $\mathfrak{G}$-orbit $\mathcal{O}_{\mathbb{C}^{*} C}$ in $m^{-1}\left(\mathbb{C}^{*} C\right)$. If a pair $(A, B) \in g l(n) \times g l(n)$ satisfies

(i) $[A, B] \in \mathbb{C}^{*} C$,

(ii) $\Sigma_{(A, B)}$ is reduced,

(iii) there is no common invariant subspace of $A$ and $B$,

then $(A, B) \in \mathcal{O}_{\mathbb{C}^{*} C}$.

Considering conjugation classes Artamkins lemma becomes

Corollary 6.1.2 Let $0 \neq C^{G L(n)} \in g l(n) / G L(n)$. There is a dense $\mathfrak{G}$-orbit $\mathcal{O}_{\mathbb{C}^{*} C^{G L(n)}}$ in $\left\{(A, B)^{G L(n)} \mid \exists k \in \mathbb{C}^{*}\right.$ such that $\left.[A, B]^{G L(n)}=k C^{G L(n)}\right\}$ containing the conjugation classes of pairs satisfying

(i) $\Sigma_{(A, B)}$ is reduced,

(ii) there is no common invariant subspace of $A$ and $B$. 
This lemma is the key for our further investigations. Since one easily obtains, that a pair $(A, B)$ corresponding to an element of $\tilde{\mathcal{M}}_{n c}^{r e g}(n)$ for $n \leq 4$ satisfies condition ( $i$ i ) of corollary 6.1.2 (see the end of the next subsection), everything of what follows is done in order to show that there is no instanton of charge $\leq 4$ having a spectral scheme, which is non reduced. This together with the examples of chapter 5.4 will show that there are exactly $n-1 \mathfrak{G}$-orbits in $\tilde{\mathcal{M}}_{n c}^{\text {reg }}(n)$ for $n \leq 4$.

\subsection{The spectral scheme}

The restriction of a locally free sheaf $E$ of rank 2 on $\mathbb{P}_{2}$ with $\left(c_{1}, c_{2}\right)=(0, n)$ to a generic line is trivial (see [OSS]). The dual of the lines, for which this is not the case, so called jumping lines, form a curve in $\mathbb{P}_{2}^{*}$, the jumping curve, which we denote by $\tilde{\Sigma}_{E}$. It is the support of the scheme $\Sigma_{E}$ called the jumping scheme defined below. We show, that the jumping curve of a locally free sheaf $E$ is the same as the spectral curve $\tilde{\Sigma}_{(A, B)}$ of the corresponding $(A, B, i, j)$, considered as a curve in $\mathbb{P}_{2}^{*}$. Therefore the spectral scheme of a locally free sheaf $E$ is non reduced if and only if the jumping scheme is. Furthermore we sketch how one shows that even $\Sigma_{E}=\Sigma_{(A, B)}$.

In chapter 5 we considered the spectral scheme as a subscheme of $\mathbb{P}_{2}$ and we chose an arbitrary coordinate ring $\mathbb{C}\left[\lambda_{0}, \lambda_{1}, \lambda_{2}\right]$, which induced homogeneous coordinates $\left(\lambda_{0}: \lambda_{1}: \lambda_{2}\right)$ on $\mathbb{P}_{2}$. But when we considered the relation between instantons an torsion free sheaves we fixed coordinates $\left(z_{0}: z_{1}: z_{2}\right)$ on $\mathbb{P}_{2}$ and we set $l_{\infty}=\left\{z_{0}=0\right\}$. Therefore we define the spectral scheme as the subscheme of $\mathbb{P}_{2}^{*}=\operatorname{Proj}\left(\mathbb{C}\left[\nu_{0}, \nu_{1}, \nu_{2}\right]\right)$ corresponding to the dual coodinates $\left(\nu_{0}: \nu_{1}: \nu_{2}\right)$ given by the ideal generated by

$$
\operatorname{det}\left(-\nu_{0} \mathbf{1}+\nu_{1} A+\nu_{2} B\right)
$$

Lemma 6.2.1 Let $E$ be a locally free sheaf of rank 2 on $\mathbb{P}_{2}$ with $\left(c_{1}, c_{2}\right)=(0, n)$ trivial at $l_{\infty}$ and $A D H M$ datum $(A, B, i, j)$ and $l$ a line in $\mathbb{P}_{2}$ such that $\check{l}=\left(\nu_{0}\right.$ : $\left.\nu_{1}: \nu_{2}\right)$. Then $l$ is a jumping line of $E$ if and only if $\operatorname{det}\left(-\nu_{0} \mathbf{1}+\nu_{1} B+\nu_{2} A\right)=0$.

\section{Proof:}

$l$ is a jumping line if and only if there is a $s \in H^{0}\left(\left.E\right|_{l}\right)$ that has a zero. In the proof of lemma 4.0.9 we have seen that there is an isomorphism $\phi: H^{0}\left(\left.E\right|_{l}\right) \rightarrow$ $H^{0}(\operatorname{ker}(b))$, an inclusion $H^{0}(\operatorname{ker}(b)) \hookrightarrow W$, and that if $\phi(s)=w \in W$ then $s$ vanishes at $p \in l$ if and only if there is a $v \in V$ such that $a_{p}(v)=w$. But for any point $q \in l$ one has $b_{q}(w)=0$, thus $s$ vanishes at $p$ if and only if 


$$
b_{q}\left(a_{p}(v)\right)=0 \forall q \in l
$$

Furthermore we have the monad condition $b_{q}\left(a_{q}(v)\right)=0$ and since $a$ and $b$ are linear in $q, 6.2 .1$ is equivalent to

$$
b_{q}\left(a_{p}(v)\right)=0 \text { for any two points } p, q \in l ; p \neq q
$$

So let $\check{l}$ be given by $\left(\nu_{0}: \nu_{1}: \nu_{2}\right)$ such that $\nu_{0} \neq 0$. Then with $p=\left(-\frac{\nu_{1}}{\nu_{0}}: 1: 0\right)$ and $q=\left(-\frac{\nu_{2}}{\nu_{0}}: 0: 1\right)$ we get

$$
\begin{aligned}
& b\left(p_{2}\right) a\left(p_{1}\right)=\left(\begin{array}{lll}
\frac{\nu_{2}}{\nu_{0}} A-\mathbf{1}, & -\frac{\nu_{2}}{\nu_{0}} B, \quad-\frac{\nu_{2}}{\nu_{0}} i
\end{array}\right)\left(\begin{array}{c}
-\frac{\nu_{1}}{\nu_{0}} B+\mathbf{1} \\
-\frac{\nu_{1}}{\nu_{0}} A \\
\frac{\nu_{1}}{\nu_{0}} j
\end{array}\right)= \\
= & -\frac{\nu_{1} \nu_{2}}{\nu_{0}^{2}} A B+\frac{\nu_{1}}{\nu_{0}} A+\frac{\nu_{2}}{\nu_{0}} B-\mathbf{1}+\frac{\nu_{1} \nu_{2}}{\nu_{0}^{2}} B A-\frac{\nu_{1} \nu_{2}}{\nu_{0}^{2}} i j= \\
= & \frac{\nu_{1} \nu_{2}}{\nu_{0}^{2}}([A, B]+i j)+\frac{\nu_{1}}{\nu_{0}} A+\frac{\nu_{2}}{\nu_{0}} B-\mathbf{1}=\frac{\nu_{1}}{\nu_{0}} A+\frac{\nu_{2}}{\nu_{0}} B-\mathbf{1}
\end{aligned}
$$

If $\nu_{0}=0$ we can choose two other points to show the result.

This completes the proof.

Consider the standard construction

$$
\begin{aligned}
& D \subset \mathbb{P}_{2} \times \mathbb{P}_{2}^{*} \stackrel{q}{\rightarrow} \mathbb{P}_{2} \\
& \downarrow p \\
& \mathbb{P}_{2}^{*}
\end{aligned}
$$

In general for a torsion free sheaf $\mathcal{F}$ the net of $\mathcal{F}$ (see [B1]), which is the second arrow in

$$
0 \rightarrow H^{1}(\mathcal{F}(-2)) \otimes \mathcal{O}_{\mathbb{P}_{2}^{*}}(-1) \rightarrow H^{1}(\mathcal{F}(-1)) \otimes \mathcal{O}_{\mathbb{P}_{2}^{*}} \rightarrow R^{1} p_{*}\left(\mathcal{O}_{D} \otimes q^{*} \mathcal{F}(-1)\right) \rightarrow 0
$$

defines the jumping scheme

$$
\Sigma_{\mathcal{F}}:=F_{i t t}\left(R^{1} p_{*}\left(\mathcal{O}_{D} \otimes q^{*} \mathcal{F}(-1)\right)\right)
$$

While the Kronecker modul of $\mathcal{F}$, which is given by $-\nu_{0} \mathbf{1}+\nu_{1} A+\nu_{2} B$, see [OSS] pp. 322 and $[\mathrm{LPH}]$, the second arrow of 


$$
0 \rightarrow H^{1}(\mathcal{F}(-2)) \otimes \mathcal{O}_{\mathbb{P}_{2}^{*}}(-1) \rightarrow H^{1}(\mathcal{F}(-1)) \otimes \mathcal{O}_{\mathbb{P}_{2}^{*}} \rightarrow \underline{\operatorname{Exp}_{p}^{1}}{ }_{p}\left(q^{*}(\mathcal{F}(1)), \mathcal{O}_{D}\right) \rightarrow 0
$$

where $\underline{\operatorname{Ext}}_{p}^{1}(-, \mathcal{H})=R^{1}(p \circ \underline{\operatorname{Hom}}(-, \mathcal{H}))$ defines the spectral scheme of the pair $(A, B)$ corresponding to $\mathcal{F}$, i.e.

$$
\Sigma_{(A, B)}=\operatorname{Fitt}_{0}\left(\underline{\operatorname{Exp}}_{p}^{1}\left(q^{*}(\mathcal{F}(1)), \mathcal{O}_{D}\right)\right)
$$

For a locally free $E$ the sheaves $R^{1} p_{*}\left(\mathcal{O}_{D} \otimes q^{*} E(-1)\right)$ and $\underline{\operatorname{Exp}}_{p}^{1}\left(q^{*}(E(1)), \mathcal{O}_{D}\right)$ are isomorphic:

The exact sequence of low degree for the Grothendieck spectral sequence of $p_{*} \circ$ $\underline{\operatorname{Hom}}\left(-, \mathcal{O}_{D}\right)$ applied to a coherent sheaf $\mathcal{H}$ is

$$
0 \rightarrow R^{1} p_{*}\left(\underline{\operatorname{Hom}}\left(\mathcal{H}, \mathcal{O}_{D}\right)\right) \rightarrow \underline{\operatorname{Ext}}_{p}^{1}\left(\mathcal{H}, \mathcal{O}_{D}\right) \rightarrow p_{*} \underline{\operatorname{Ext}}^{1}\left(\mathcal{H}, \mathcal{O}_{D}\right) \rightarrow
$$

But for a locally free $E, q^{*}(E(1))$ is locally free as well and therefore

$$
\underline{\operatorname{Ext}}^{1}\left(q^{*}(E(1)), \mathcal{O}_{D}\right)=\underline{\operatorname{Ext}}^{1}\left(\mathcal{O}, \mathcal{O}_{D}\right) \otimes\left(q^{*}(E(1))\right)^{*}=0
$$

because $\underline{\operatorname{Ext}} t^{1}\left(\mathcal{O}, \mathcal{O}_{D}\right)=0$, which one deduces from applying $\underline{\operatorname{Hom}}(\mathcal{O},-)$ to the resolution of $D$

$$
0 \rightarrow \mathcal{O}_{\mathbb{P}_{2} \times \mathbb{P}_{2}^{*}}(-1,-1) \stackrel{s_{D}}{\rightarrow} \mathcal{O}_{\mathbb{P}_{2} \times \mathbb{P}_{2}^{*}} \rightarrow \mathcal{O}_{D} \rightarrow 0
$$

Therefore

$$
\underline{\operatorname{Ext}}_{p}^{1}\left(q^{*}(E(1)), \mathcal{O}_{D}\right) \cong R^{1} p_{*}\left(\underline{\operatorname{Hom}}\left(q^{*}(E(1)), \mathcal{O}_{D}\right)\right) \cong R^{1} p_{*}\left(q^{*}(E(-1)) \otimes \mathcal{O}_{D}\right)
$$

since $E$ is selfdual in this case. Thus for a locally free sheaf $E$ the two sheaves are isomorphic and therefore the net and the Kronecker module of $E$ are the same. For a not locally free $\mathcal{F}$ these sheaves do not have to be isomorphic but since we have shown that setsupp $\left.\left(\underline{\operatorname{Ext}}_{p}^{1}\left(q^{*}(\mathcal{F}(1)), \mathcal{O}_{D}\right)\right)\right)=\operatorname{set} \operatorname{supp}\left(R^{1} p_{*}\left(q^{*}(\mathcal{F}(-1)) \otimes\right.\right.$ $\left.\left.\mathcal{O}_{D}\right)\right)$ ) and $R^{1} p_{*}\left(q^{*}(\mathcal{F}(-1)) \otimes \mathcal{O}_{D}\right)$ and $\underline{\operatorname{Ext}}_{p}^{1}\left(q^{*}(\mathcal{F}(1)), \mathcal{O}_{D}\right)$ have the same Chern class, one has

$$
\operatorname{Fitt}_{0}\left(R^{1} p_{*}\left(q^{*}(\mathcal{F}(-1)) \otimes \mathcal{O}_{D}\right)\right)=\operatorname{Fitt}_{0}\left(\underline{\operatorname{Exp}}_{p}^{1}\left(q^{*}(\mathcal{F}(1)), \mathcal{O}_{D}\right)\right)
$$


see $[\mathrm{LPH}]$.

The net of a locally free $E \in \mathfrak{M}(4)$, which in our notation is $(A, B, \mathbf{1})$ has no invariant subspace of dimension or codimension 1 (see for example [A2], thm. 1 ). Therefore for $n \leq 3$ condition ( $i i)$ of corollary 6.1.2 is trivially satisfied. For $n=4$ the only possibility for a pair $(A, B)$ corresponding to $E$ not to satisfy $(i i)$ of corollary 6.1 .2 would be to have a two dimension invariant subspace, i.e.

$$
A=\left(\begin{array}{cc}
A_{1} & A_{2} \\
0 & A_{3}
\end{array}\right), \quad B=\left(\begin{array}{cc}
B_{1} & B_{2} \\
0 & B_{3}
\end{array}\right)
$$

with $A_{k}, B_{k} 2 \times 2$ matrices. The commutator $[A, B]=C$ is also of such a form, and since $C=-i j$ stability implies that $r k\left(C_{1}\right)=r k\left(C_{3}\right)=1$. Considering $A_{1}$ in Jordan normal form the equation $C_{1}=\left[A_{1}, B_{1}\right]$ together with $r k\left(C_{1}\right)=1$ induces that $B_{1}$ is also upper triangular. Therefore we would have a invariant subspace of dimension one, a contradiction. So a pair $(A, B)$ representing an element of $\mathcal{M}_{n c}^{r e g}(4)$ satisfies condition (ii) of corollary 6.1 .2 and we only have to investigate whether there are elements of $\mathcal{M}_{n c}^{r e g}(n)$ for $n \leq 4$, that have a non reduced spectral scheme.

\subsection{Spectral schemes of elements of $\tilde{\mathcal{M}}_{n c}^{r e g}(2)$ and $\tilde{\mathcal{M}}_{n c}^{\text {reg }}(3)$}

Now we will show, that there is one $\mathfrak{G}$-orbit in $\mathcal{M}_{n c}^{r e g}(2)$ and two orbits in $\mathcal{M}_{n c}^{\text {reg }}(3)$. To do that, we need the following lemma:

Lemma 6.3.1 Let $\mathcal{F}$ be a coherent sheaf on $\mathbb{P}_{2}$ that is the quotient of two locally free sheaves $L_{1}, L_{2}$ of rank $k$ :

$$
0 \rightarrow L_{1} \stackrel{\phi}{\rightarrow} L_{2} \rightarrow \mathcal{F} \rightarrow 0
$$

Let $p \in \operatorname{supp}\left(\operatorname{Fitt}_{0}(\mathcal{F})\right)$ but $p \notin \operatorname{supp}\left(F i t t_{1}(\mathcal{F})\right)$ and $U \simeq \mathbb{C}^{2}$ an open neighborhood of $p$ with coordinates $\left(x_{1}, x_{2}\right)$ and $p=(0,0)$. Then Fitt $t_{0}(\mathcal{F})$ is singular at $p$ if and only if

$$
\frac{\partial \phi}{\partial x_{i}}(p)(\operatorname{ker} \phi(p)) \subset i m(\phi(p)) \text { for } i=1,2
$$

\section{Proof:}


One has $\operatorname{codim}(\mathcal{F})=1$, because applying $\underline{\operatorname{Hom}}(-, \mathcal{O}(-3))$ to 6.3 .1 gives $\underline{\operatorname{Ext}} t^{2}(\mathcal{F}, \mathcal{O}(-3))=0$, which is due to 2.4 .1 a contradiction to $\operatorname{codim}(\mathcal{F})=2$. So $F_{i t t_{0}}(\mathcal{F})$ is singular at $p$ if and only if $T_{F i t t_{0}(\mathcal{F}), p}$ is of dimension 2 . Let $U=\operatorname{Spec}\left(\mathbb{C}\left[x_{1}, x_{2}\right]\right)$. Then $p$ corresponds to the maximal ideal $\left(x_{1}, x_{2}\right)$ and $T_{\text {Fitt }_{0}(\mathcal{F}), p}^{*}$ is isomorphic to $\left(x_{1}, x_{2}\right) /\left(x_{1}, x_{2}\right)^{2}$ as a $\mathbb{C}$ vector space. A subscheme of $U$ given by an ideal $(f)$ with $f \in \mathbb{C}\left[x_{1}, x_{2}\right]$ is singular a $p$ if and only if $f \in\left(x_{1}, x_{2}\right)^{2}$, therefore $\operatorname{dim}_{\mathbb{C}}\left(T_{F_{i t t}(\mathcal{F}), p}\right)=2$ if and only if

$$
\frac{\partial \operatorname{det}(\phi)}{\partial x_{i}}(p)=0 \quad \text { for } i=1,2
$$

For $i=1$ this means

$$
\operatorname{det}(\phi)(\epsilon, 0)=\mathcal{O}\left(\epsilon^{2}\right)
$$

for infinitesimal $\epsilon$. Since $p \notin \operatorname{supp}\left(F_{i t t}(\mathcal{F})\right)$ one has $r k(\phi(p))=k-1$. Therefore 6.3 .2 is equivalent to

$$
\exists v \in V \text { s.t } \phi(\epsilon, 0) v=\mathcal{O}\left(\epsilon^{2}\right)
$$

where we identify the fibers of $L_{1}$ and $L_{2}$ at $\mathrm{p}$ with the vector space $V$. Let $v=u+\epsilon \tilde{u}$ with $u \in \operatorname{ker}(\phi(p))$ then 6.3 .3 becomes

$$
\phi(\epsilon, 0)(u+\epsilon \tilde{u})=\phi(0,0) u+\epsilon \phi(0,0) \tilde{u}+\epsilon \frac{\partial \phi}{\partial x_{1}}(0,0) u+O\left(\epsilon^{2}\right)
$$

So 6.3.3 is satisfied if and only if $\frac{\partial \phi}{\partial x_{1}}(p)(\operatorname{ker} \phi(p)) \subset i m(\phi(p))$. The same is true for $i=2$

This completes the proof.

Proposition 6.3.2 The action of $\mathfrak{G}$ on $\tilde{\mathcal{M}}_{n c}^{r e g}(2)$ is transitive.

\section{Proof:}

$a \in \mathcal{A}_{2}$ maps a non reduced component to a non reduced component and $\mathcal{A}_{2}$ acts transitive on the set of lines in $\mathbb{P}_{2}$, therefore we can assume that the non reduced spectral scheme is given by $\left(\nu_{0}^{2}\right)$. That means $r k\left(\nu_{1} A+\nu_{2} B\right) \leq 1 \forall\left(\nu_{1}, \nu_{2}\right) \neq(0,0)$, and since $r k(A)=0$ or $r k(B)=0$ would be a contradiction to $[A, B] \neq 0$ we have $r k(A)=r k(B)=1$. Therefore the points $(0: 1: 0)$ and $(0: 0: 1)$ do not belong to $\operatorname{Fitt}_{1}(\mathcal{F})$, where $\mathcal{F}$ is the sheaf corresponding to the instanton 
uniquely defined by $(A, B)$, so we can apply lemma 6.3 .1 at these 2 points. With $\phi=-\nu_{0} \mathbf{1}+\nu_{1} A+\nu_{2} B$ we get for $p=(0: 0: 1)$

$$
\frac{d \phi}{d \nu_{1}}=A \quad \frac{d \phi}{d \nu_{0}}=-\mathbf{1}
$$

and for $p=(0: 1: 0)$

$$
\frac{d \phi}{d \nu_{2}}=B \quad \frac{d \phi}{d \nu_{0}}=-\mathbf{1}
$$

Since $\frac{d \phi}{d \nu_{0}}=\mathbf{- 1}$ lemma 6.3.1 induces $\operatorname{ker}(A) \subset i m(A)$. So with a basis such that $\operatorname{span}\left\{e_{1}\right\}=\operatorname{ker}(A)$ and $\frac{d \phi}{d \nu_{2}}=B$ we get

$$
A=\left(\begin{array}{cc}
0 & * \\
0 & 0
\end{array}\right), \quad B=\left(\begin{array}{cc}
* & * \\
0 & *
\end{array}\right)
$$

Which is a contradiction to $\operatorname{rk}[A, B]=2$.

This completes the proof.

Proposition 6.3.3 There are two $\mathfrak{G}$-orbits in $\tilde{\mathcal{M}}_{n c}^{r e g}(3)$.

\section{Proof:}

As in the proof of lemma 6.3.2 we can assume that the non reduced component of the spectral scheme is given by the ideal $\left(\nu_{0}^{2}\right)$, so $r k\left(\nu_{1} A+\nu_{2} B\right) \leq 2 \forall\left(\nu_{1}, \nu_{2}\right) \neq$ $(0,0)$. $r k\left(\nu_{1} A+\nu_{2} B\right) \leq 1 \forall\left(\nu_{1}, \nu_{2}\right) \neq(0,0)$ is impossible since $r k[A, B]=2$, $r k\left(\nu_{1} A+\nu_{2} B\right)=2$ for a generic $\left(\nu_{1}, \nu_{2}\right)$. The subgroup of $\mathcal{A}_{2}$ that preserves the line $\nu_{2}=0$ moves the points on this line, therefore we can assume $r k(A)=$ $r k(B)=2$. This means that the points $(0: 1: 0)$ and $(0: 0: 1)$ do not belong to $F_{i t t}(\mathcal{F})$, therefore we can apply lemma 6.3 .1 at these 2 points. One again with $\phi=-\nu_{0} \mathbf{1}+\nu_{1} A+\nu_{2} B$ we get

$$
\frac{d \phi}{d \nu_{1}}=A \quad \frac{d \phi}{d \nu_{2}}=B \quad \frac{d \phi}{d \nu_{2}}=\mathbf{- 1}
$$

Since $\frac{d \phi}{d \nu_{0}}=\mathbf{- 1}$ lemma 6.3.1 induces $\operatorname{ker}(A) \subset i m(A)$ and $\operatorname{ker}(B) \subset i m(B)$. We show, that a regular instanton with $\operatorname{Proj}\left(\mathbb{C}\left[\nu_{0}, \nu_{1}, \nu_{2}\right] /\left(\nu_{0}^{2}\right)\right) \subset \Sigma_{(A, B)}$ does not exist: We have to consider 2 cases.

1. $\operatorname{ker}(B) \subset \operatorname{im}(A)$ : 
- $\operatorname{ker}(B)=\operatorname{ker}(A)$

We choose a basis of $V$ such that $\operatorname{span}\left\{e_{1}\right\}=\operatorname{ker}(A)$ and $\operatorname{span}\left\{e_{1}, e_{2}\right\}=i m(A)$ : Thus

$$
A=\left(\begin{array}{lll}
0 & * & * \\
0 & * & * \\
0 & 0 & 0
\end{array}\right) \quad B=\left(\begin{array}{lll}
0 & * & * \\
0 & * & * \\
0 & * & *
\end{array}\right)
$$

then

$$
[A, B]=\left(\begin{array}{ccc}
0 & * & * \\
0 & * & * \\
0 & * & *
\end{array}\right)
$$

But this means, that $j_{1}=0$. Therefore by setting $S=\operatorname{span}\left\{e_{1}\right\}$ the stability condition (S2) is violated.

- $\operatorname{ker}(b) \neq \operatorname{ker}(A)$ :

Then we choose $e_{1}, e_{2}$ such that $\operatorname{span}\left\{e_{1}\right\}=\operatorname{ker}(A), \operatorname{span}\left\{e_{2}\right\}=$ $\operatorname{ker}(B)$ and $\operatorname{span}\left\{e_{1}, e_{2}\right\}=i m(A)$. Since $\frac{d \phi}{d \nu_{2}}=B$ we need $b_{31}=0$ due to lemma 6.3.1:

$$
A=\left(\begin{array}{lll}
0 & * & * \\
0 & * & * \\
0 & 0 & 0
\end{array}\right) \quad B=\left(\begin{array}{ccc}
* & 0 & * \\
* & 0 & * \\
0 & 0 & *
\end{array}\right)
$$

then

$$
[A, B]=\left(\begin{array}{ccc}
* & * & * \\
* & * & * \\
0 & 0 & 0
\end{array}\right)
$$

But this means, that $i_{3}=0$. Therefore by setting $S=\operatorname{span}\left\{e_{1}, e_{2}\right\}$ the stability condition $(\mathbf{S 1})$ is violated.

2. $\operatorname{ker}(B) \nsubseteq \operatorname{im}(A)$ :

Then we choose a basis such that $\operatorname{span}\left\{e_{1}\right\}=\operatorname{ker}(A), \operatorname{span}\left\{e_{3}\right\}=\operatorname{ker}(B)$ and $\operatorname{span}\left\{e_{1}, e_{2}\right\}=i m(A)$.

$$
A=\left(\begin{array}{ccc}
0 & * & * \\
0 & * & * \\
0 & 0 & 0
\end{array}\right) \quad B=\left(\begin{array}{ccc}
* & * & 0 \\
* & * & 0 \\
0 & * & 0
\end{array}\right)
$$


We compute the generator of the Fitting ideal:

$$
\begin{aligned}
\operatorname{det}(\phi)= & \nu_{0}\left(\nu_{0}+\nu_{2} b_{11}\right)\left(\nu_{0}+\nu_{2} b_{22}+\nu_{1} a_{22}\right)+\nu_{1} \nu_{2}^{2} a_{13} b_{21} b_{32} \\
& -\left(\nu_{0}+\nu_{2} b_{11}\right) \nu_{1} \nu_{2} a_{23} b_{32}-\nu_{0} \nu_{2} b_{21}\left(\nu_{2} b_{12}+\nu_{1} a_{12}\right)
\end{aligned}
$$

$\operatorname{ker}(B) \subset i m(B)$ implies $b_{32} \neq 0$. To get a spectral scheme with component given by $\left(\nu_{0}^{2}\right)$ the first summand of 6.3 .5 must have a factor $\nu_{0}^{2}$ and the other summands have to vanish. There are two possibilities for a factor $\nu_{0}^{2}$ in $\nu_{0}\left(\nu_{0}+\nu_{2} b_{11}\right)\left(\nu_{0}+\nu_{2} b_{22}+\nu_{1} a_{22}\right)$ :

- $b_{11}=0$ :

The third summand of 6.3 .5 vanishes only if $a_{23}=0$. Therefore the second summand of 6.3 .5 vanishes if $a_{13} b_{21}=0$. But if $b_{21}=0$ then $r k(B)<2$, and if $a_{13}=0$ then $r k(A)<2$.

- $b_{22}=a_{22}=0$ :

The last three summands again only vanish if $a_{23}=0$ and $a_{13} b_{21}=0$ and one gets the same contradiction as before.

This completes the proof. 


\section{Chapter 7}

\section{Stability of locally free sheaves corresponding to instantons}

\subsection{Stability of torsion free sheaves}

We will introduce the notation of $\mu$ - and $G$ - (semi)stability for a torsion free sheaf on $\mathbb{P}_{m}$ and show, that in the case $\mathbb{P}_{2}$ for a locally free $E$ of rank 2 with $\left(c_{1}(E), c_{2}(E)\right)=(0, n)$, (which means, that $E$ corresponds to a regular instanton due to lemma 4.0.10) the conditions $r k[A, B]=2, \mu$-stable and $G$-semistable are equivalent.

\subsubsection{Stability on curves}

Consider a smooth plane curve $C$ and let $\mathcal{F}$ be a torsion free and therefore locally free sheaf on it, then the degree of $\mathcal{F}$ is defined as

$$
d(\mathcal{F}):=\chi(\mathcal{F})-r k(\mathcal{F}) \cdot \chi\left(\mathcal{O}_{C}\right)
$$

This just extends the definition of the degree of a line bundle on $C$, since for a given divisor $D$ one has $d\left(L_{D}\right)=\operatorname{deg}(D)$

Definition 7.1.1 In the above setting we define the slope of $\mathcal{F}$ :

$$
\mu(\mathcal{F}):=\frac{d(\mathcal{F})}{r k(\mathcal{F})}
$$

$\mathcal{F}$ is called (semi)stable if and only if for any coherent $\mathcal{F}^{\prime} \subset \mathcal{F}$ one has

$$
\mu\left(\mathcal{F}^{\prime}\right)<\mu(\mathcal{F}) \text { resp. } \mu\left(\mathcal{F}^{\prime}\right) \leq \mu(\mathcal{F})
$$


We can express this in terms of the Hilbert polynomial $P_{\mathcal{F}}(n):=\chi(\mathcal{F}(n))$. On the curve one has

$$
P_{\mathcal{F}}(n)=r k(\mathcal{F}) \cdot \operatorname{deg} C \cdot n+\chi(\mathcal{F})
$$

Therefore

$$
\begin{aligned}
0 \leq \mu(\mathcal{F})-\mu\left(\mathcal{F}^{\prime}\right) & =\frac{\chi(\mathcal{F})}{r k(\mathcal{F})}-\chi\left(\mathcal{O}_{C}\right)-\left(\frac{\chi\left(\mathcal{F}^{\prime}\right)}{r k\left(\mathcal{F}^{\prime}\right)}-\chi\left(\mathcal{O}_{C}\right)\right)=\frac{\chi(\mathcal{F})}{r k(\mathcal{F})}-\frac{\chi\left(\mathcal{F}^{\prime}\right)}{r k\left(\mathcal{F}^{\prime}\right)} \pm \operatorname{deg} C \cdot n= \\
& \frac{P_{\mathcal{F}}(n)}{r k \mathcal{F}}-\frac{P_{\mathcal{F}^{\prime}}(n)}{r k \mathcal{F}^{\prime}}:=p_{\mathcal{F}}(n)-p_{\mathcal{F}^{\prime}}(n)
\end{aligned}
$$

where $p_{\mathcal{F}}(n)$ is the reduced Hilbert polynomial of $\mathcal{F}$. Thus the semistability condition is equivalent to

$$
p_{\mathcal{F}^{\prime}}(n) \leq p_{\mathcal{F}}(n)
$$

\subsubsection{Stability on $\mathbb{P}_{m}$}

Because of that two equivalent conditions for stability on curves there are two kinds of generalizations of stability for a torsion free sheaf $\mathcal{F}$ on $\mathbb{P}_{2}$ :

(1) $\mu$-semistability: $\mu\left(\mathcal{F}^{\prime}\right) \leq \mu(\mathcal{F})$ for any coherent $\mathcal{F}^{\prime} \subset \mathcal{F}$.

(2) $G$-semistability: i.e. $p_{\mathcal{F}^{\prime}}(n) \leq p_{\mathcal{F}}(n)$ for any coherent $\mathcal{F}^{\prime} \subset \mathcal{F}$.

where we define $\mu(\mathcal{F}):=\frac{c_{1}(\mathcal{F})}{r k(\mathcal{F})}$, since $d\left(\left.\mathcal{F}\right|_{l}\right)=c_{1}(\mathcal{F})$ for a general line $l \subset \mathbb{P}_{2}$. These are also the definitions of stability on $\mathbb{P}_{2}$. The reduced Hilbert polynomial for a sheaf $\mathcal{F}$ on $\mathbb{P}_{2}$ reads

$$
p_{\mathcal{F}}(n)=\tau_{1} n^{2}+\left(\mu(\mathcal{F})+\tau_{2}\right) n+\chi(\mathcal{F})
$$

with constants $\tau_{1}, \tau_{2}$ (see [LP3]). Thus we can easily compare these two stabilities:

$$
\begin{array}{ccc}
\underset{\uparrow}{G \text {-semistable }} & \Rightarrow & \mu \text {-semistable } \\
\underset{\uparrow}{G \text {-stable }} & \Leftarrow & \mu \text {-stable }
\end{array}
$$

Furthermore there is a characterization of $\mu$-(semi)stability for sheaves of rank 2 on $\mathbb{P}_{n}$ by cohomology: For a sheaf $\mathcal{F}$ set $k_{\mathcal{F}}$ s.t. $c_{1}\left(\mathcal{F}\left(k_{\mathcal{F}}\right)\right)$ is 0 or -1 . We call $\mathcal{F}\left(k_{\mathcal{F}}\right)$ the nomalization of $\mathcal{F}$.

Lemma 7.1.2 ([OSS] Ch.2, Lemma 1.2.5) Let $\mathcal{F}$ be a torsion free sheaf of rank 2 on $\mathbb{P}_{n}$. Consider the conditions

1. $\mathcal{F}$ is $\mu$-stable. 
2. $H^{0}\left(\mathcal{F}_{\text {norm }}\right)=0$.

Then we have the implication 1) $\Rightarrow 2)$. If $\mathcal{F}$ is furthermore reflexive one also has 2) $\Rightarrow 1$ ). The same is true for the conditions

1. $\mathcal{F}$ is $\mu$-semistable.

2. $H^{0}\left(\mathcal{F}_{\text {norm }}(-1)\right)=0$.

Lemma 7.1.3 For a torsion free sheaf $\mathcal{F}$ of rank 2 on $\mathbb{P}_{2}$ with $\left(c_{1}, c_{2}\right)=(0, n)$ and corresponding tuple $(A, B, i, j)$ one has $r k[A, B]=2$ if and only if $H^{0}(\mathcal{F})=$ 0 .

\section{Proof:}

From the sequence (which comes from the monad of $\mathcal{F}$ )

$$
0 \rightarrow V \otimes \mathcal{O}(-1) \rightarrow \operatorname{ker} b \rightarrow \mathcal{F} \rightarrow 0
$$

we get since $H^{0}(\mathcal{O}(-1))=H^{1}(\mathcal{O}(-1))=0$

$$
H^{0}(\operatorname{ker} b) \cong H^{0}(\mathcal{F})
$$

Applying the cohomology functor to

$$
0 \rightarrow \operatorname{ker} b \rightarrow W \otimes \mathcal{O} \stackrel{b}{\rightarrow} V \otimes \mathcal{O}(1) \rightarrow 0
$$

we get

$$
0 \rightarrow H^{0}(\operatorname{ker} b) \rightarrow H^{0}(W \otimes \mathcal{O}) \stackrel{b_{*}}{\rightarrow} H^{0}(V \otimes \mathcal{O}(1))
$$

Thus $H^{0}(\operatorname{ker} b)=H^{0}(\mathcal{F})=0$ if and only if $b_{*}$ is injective. With the notation of the proof of 4.0.9, $W=V \oplus V \oplus \tilde{W}, b=\sum z_{i} b_{i}, H^{0}(W \otimes \mathcal{O})=W$ and $H^{0}(V \otimes \mathcal{O}(1))=V \otimes H^{0}(\mathcal{O}(1))$ we get

$$
b_{*}\left(v_{1} \oplus v_{2} \oplus w\right)=\left(B v_{2}-A v_{1}+i w\right) \otimes z_{0}+v_{2} \otimes z_{1}-v_{1} \otimes z_{2}
$$

Thus $b_{*}$ is not injective if and only if there is a $w \in \tilde{W}$ such that $i w=0$. But that means, that $r k(i)<2$, which implies $r k(i j)<2$ and therefore $r k[A, B]<2$ if and only if $H^{0}(\mathcal{F}) \neq 0$.

This completes the proof. 
Lemma 7.1.4 [OSS] Let $E$ be a locally free sheaf of rank 2 on $\mathbb{P}_{2}$ with $\left(c_{1}, c_{2}\right)=$ $(0, n)$ with $n>0$. Then $E$ is $\mu$-stable if and only if it is $G$-semistable.

Proof: We only have to show, that $G$-semistability induces $\mu$-stability. For any $\mathcal{F} \in \tilde{\mathfrak{M}}(n)$ one has $H^{0}(E(-1))=0$. Suppose $E$ is not $\mu$-stable, i.e. $H^{0}(E) \neq 0$. Let $s$ be a global section of $E$. Since $H^{0}(E(-1))=0$, Lemma 2 of [B1] implies, that the zeros of $s$ must be of codim. 0 or 2 . Since $E$ is locally free an almost everywhere vanishing section must vanish identically. Therefore we only have to consider codim. 2. Set $Y:=(s)_{0}$. We have

$$
0 \rightarrow \mathcal{O} \stackrel{s}{\rightarrow} E \rightarrow \mathcal{K} \rightarrow 0
$$

If $Y$ is empty then the restriction of 7.1.1 to any point $p \in \mathbb{P}_{2}$ is exact, which means $\operatorname{Tor}^{1}\left(\mathcal{K}, \mathcal{O}_{p}\right)=0$, thus by lemma 2.5.1 $\mathcal{K}$ is locally free: $\mathcal{K}=\mathcal{O}(i)$. But from 7.1.1 we get as well $\operatorname{ch}(\mathcal{K})=1-n^{2} h^{2}$ which can only be satisfied with $i=n=0$. This contradicts the assumption $n>0$.

If $Y$ is not empty, we get $\mathcal{K}=\mathcal{J}_{Y}$, the ideal sheaf of $Y$. Then the $G$-semistability gives:

$$
\begin{gathered}
\chi(\mathcal{O}(k))=P_{\mathcal{O}}(k) \leq \frac{P_{E}(k)}{2} \leq P_{\mathcal{J}_{Y}}(k)=\chi\left(\mathcal{J}_{Y}(k)\right)=\chi(\mathcal{O}(k))-\chi\left(\mathcal{O}_{Y}(k)\right) \\
\Rightarrow \chi\left(\mathcal{O}_{Y}(k)\right) \leq 0
\end{gathered}
$$

But for $k \gg 0$ this is $H^{0}\left(\mathcal{O}_{Y}(k)\right)$ due to Serres vanishing theorem, and due to his theorem $\mathrm{A} \mathcal{O}_{Y}(k)$ is then generated by global sections, which implies the contradiction $H^{0}\left(\mathcal{O}_{Y}(k)\right)>0$.

This completes the proof.

Corollary 7.1.5 There are bijections between the following sets:

- Locally free $G$-semistable sheaves of rank 2 on $\mathbb{P}_{2}$ with $\left(c_{1}, c_{2}\right)=(0, n)$ trivial at $l_{\infty}$.

- Locally free $\mu$-stable sheaves of rank 2 on $\mathbb{P}_{2}$ with $\left(c_{1}, c_{2}\right)=(0, n)$ trivial at $l_{\infty}$

- $\tilde{\mathcal{M}}_{n c}^{r e g}(n)$ 


\section{$7.2 \mathfrak{G}$ and the Cremora group}

Let $C r\left(\mathbb{P}_{2}\right)$ denote the group of biregular automorphism of $\mathbb{P}_{2}$ with homogeneous coordinates $\left(z_{0}: z_{1}: z_{2}\right)$. Under the identification $\mathbb{C}^{2}=\left\{\left(1: z_{1}: z_{2}\right)\right\}$ with affine coordinates $(x, y)$ the elements of $\mathfrak{G}$ uniquely extend to elements of $\mathrm{Cr}\left(\mathbb{P}_{2}\right)$ in the following way:

$$
\begin{gathered}
\left((x, y) \mapsto\left(\mu_{0} x+\mu_{1} y+\mu_{2}, \nu_{0} x+\nu_{1} y+\nu_{2}\right)\right) \rightarrow\left(\begin{array}{ccc}
1 & 0 & 0 \\
\mu_{2} & \mu_{0} & \mu_{1} \\
\nu_{2} & \nu_{0} & \nu_{1}
\end{array}\right) \in \operatorname{PGL}(3) \subset C r\left(\mathbb{P}_{2}\right) \\
\left((x, y) \mapsto\left(x, y+x^{m}\right)\right) \rightarrow\left(\left(z_{0}: z_{1}: z_{2}\right) \mapsto\left(z_{0}^{m}: z_{0}^{m-1} z_{1}: z_{0}^{m-1} z_{2}+z_{1}^{m}\right)\right)
\end{gathered}
$$

Therefore one can consider $\mathfrak{G}$ as a subgroup of $C r\left(\mathbb{P}_{2}\right)$. Artamkin shows in [A2] the following:

Theorem 7.2.1 There is a well defined action of $\mathfrak{G} \subset C r\left(\mathbb{P}_{2}\right)$ on the set of $\mu$ stable locally free sheaves $E$ with $c_{1}(E)=0, c_{2}(E)=n$ trivial at $l_{\infty}$. The bijection between $\mu$-stable locally free sheaves and its Kronecker modules given by theorem 4.0.5 is $\mathfrak{G}$-equivariant.

We give a sketch of the proof: Since $a \in \mathcal{A}_{2}$ is a bijection of $\mathbb{P}_{2}$ preserving $l_{\infty}$, $a_{*}(E)$ is well defined and locally free. Since $a$ is just a coordinate transformation a look at the monad of $E$, that defines $A, B$ shows the $\mathcal{A}_{2}$-equivariance.

The non trivial part is the $\mathfrak{E}$-action. $e_{x^{m}}$ is not a bijection of $\mathbb{P}_{2}$, therefore we cannot consider the push forward of $E$ under $e_{x^{m}}$. But since it is a birational map, there is a projective surface $X$ and a diagram

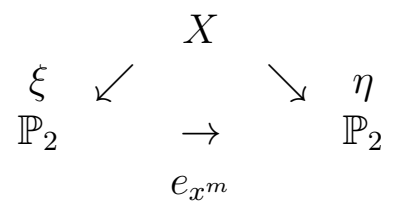

with regular maps $\xi, \eta$ (See [Sh2],p.210). Thus one defines $\left(e_{x^{m}}\right)_{*}(E):=$ $\eta_{*}\left(\xi^{*}(E)\right) . \xi$ consists of the following $2 m-1 \sigma$-processes: 1 . blow up the point $(0: 0: 1)$, denote the exceptional divisor by $C_{1} .2$. blow up the intersection point of $C_{1}$ and the proper preimage of $l_{\infty}$ with exceptional divisor $C_{2}$. 3. blow up the intersection point of $C_{2}$ and the proper preimage of $C_{1} \ldots \mathrm{m}$. blow up: blow up the intersection point of $C_{m-1}$ and the proper preimage of $C_{1}$. Let $\tilde{X}$ be the 
surface resulting from this $m \sigma$-processes and $D$ the zero set divisor of the map $\tilde{X} \rightarrow \mathbb{P}_{2}$ resulting from $\left(z_{0}: z_{1}: z_{2}\right) \mapsto z_{0}^{m-1} z_{2}+z_{1}^{m}$. The following $\sigma$-processes are blow ups of $D$ resp. its proper preimage and the exceptional divisor of the blow up before.

$\eta$ consists of the blow downs of the preimages of the following divisors in the following order: $l_{\infty}, C_{1}, C_{2}, \ldots, C_{2 m-2}$. Since the blow ups and blow downs commute one can perform after the first blow up, which makes $\mathbb{P}_{2}$ to the rational ruled surface $F_{1}$ the second blow up followed by the first blow down, which leads to $F_{2}$. In this way one can factor $e_{x^{m}}$ through $F_{m}$ :

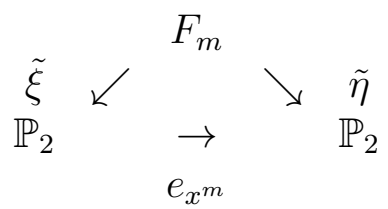

with regular maps $\tilde{\xi}, \tilde{\eta}$ and $\left(e_{x^{m}}\right)_{*}(E)=\tilde{\xi}_{*}\left(\tilde{\eta}^{*}(E)\right)$. Let $\psi_{i}: F_{i-1} \rightarrow F_{i}$ be the birational map resulting from a blow up blow down process. Now Artamkin shows that there is an isomorphism between the set of stable bundles on $F_{i}$ and $F_{i-1}$ respectively the stable bundles on $F_{1}$ and $\mathbb{P}_{2}$ trivial on certain divisors, and a 1-to-1 correspondence of those stable bundles and stable nets, which are linear maps

$$
V \otimes H^{0}\left(\mathcal{O}_{F_{i}}(C+(i) f)\right) \rightarrow V^{*}
$$

with $n$ dimensional vector spaces $V, V^{*}$ and $C, f$ being generators of the Picard group of $F_{i}$. Furthermore he shows that under the natural inclusion $H^{0}\left(\mathcal{O}_{F_{i-1}}(C+(i-1) f)\right) \hookrightarrow H^{0}\left(\mathcal{O}_{F_{i}}(C+i f)\right)$ for a stable bundle $E$ on $F_{i-1}$ with net $\left(\gamma_{0}, \gamma_{1}, \gamma_{2}\right)$ the restriction of the net of $\left(\psi_{i}\right)_{*}(E)$ to $H^{0}\left(\mathcal{O}_{F_{i-1}}(C+(i-1) f)\right)$ and the net of $E$ coincide and that the net of $\left(\psi_{i}\right)_{*}(E)$ is uniquely determined by its restriction to $H^{0}\left(\mathcal{O}_{F_{i-1}}(C+(i-1) f)\right)$.

Because of these facts and the circumstance that the lines $z_{0}=0$ and $z_{1}=0$ are invariant under the resolution of $e_{x}^{m}$ one get $e_{x^{m}}\left(\gamma_{0}\right)=\gamma_{0}=\mathbf{1}$ and $e_{x^{m}}\left(\gamma_{1}\right)=\gamma_{1}=$ A. $e_{x^{m}}\left(\gamma_{2}\right)$ is obtained in the following way: One has $\eta^{*}\left(S_{z_{2}}\right) \nsubseteq \xi^{*}\left(H^{0}\left(\mathcal{O}_{\mathbb{P}_{2}}(1)\right)\right)$, where $S_{z_{2}} \subset H^{0}\left(\mathcal{O}_{\mathbb{P}_{2}}(1)\right)$ consist of the section with $\left\{z_{2}=0\right\}$ as zero set divisor. But for a representative $s_{2} \in S_{z_{2}}$ one gets $\eta^{*}(s)=\xi^{*}(s)+t$ where $t \in H^{0}\left(\mathcal{O}_{F_{i}}(C+\right.$ $(i) f)$ ) has a zero set divisor of the form $C+m f_{2}$. Furthermore for $s_{1} \in S_{z_{1}}$ the zero set divisor of $\xi^{*}\left(s_{1}\right)$ is $C+m f_{1}$, where $f_{1}$ and $f_{2}$ are two different fibers of $F_{m}$, and $\xi^{*}(E)$ is trivial on $f_{1}$. Now Artamkin shows that under this circumstances one has $\gamma_{C+m f_{2}}=\gamma_{C+m f_{1}}^{m}=A^{m}$ (see [A2] Prop. 5). Therefore $e_{x^{m}}\left(\gamma_{2}\right)=B+A^{m}$, which shows the equivariance of the $\mathfrak{E}$-action. 
Remark: It seems that this $\mathfrak{G}$-equivariance extends to the whole $\tilde{\mathfrak{M}}(n)$. To prove this, one has to ensure, that for torsion free sheaves on $F_{i}$ trivial on the exceptional divisors of the blow ups and blow downs one has a monad uniquely determined by a datum $(A, B, i, j)$ (for locally free sheaves those monads have been investigated in $[\mathrm{Bu}]$ ) and that even for non stable nets the formula of [A2] Prop. 5 is true. 


\title{
Chapter 8
}

\author{
$\tilde{\mathcal{M}}_{n c}^{r e g}(4)$
}

In this chapter we will show, that there is no element in $\tilde{\mathcal{M}}_{n c}^{r e g}(4)$ that has a non reduced spectral scheme. In order to do that, we will consider the space of certain coherent systems, which is a blow up of $\mathfrak{M}_{\text {Gss }}(4)$ the set of $G$-semistable torsion free sheaves with $\left(c_{1}, c_{2}\right)=(0,4)$, which includes under the identification of theorem 4.0.5 $\tilde{\mathcal{M}}_{n c}^{r e g}(4)$.

\subsection{Coherent systems}

We give a summary of coherent systems and coherent cosystems without proofs. They were introduced by LePotier in [LP1].

Definition 8.1.1 $A$ coherent system $(\Gamma, \mathcal{F})$ on $\mathbb{P}_{n}$ of dimension $d$ consists of a coherent sheaf $\mathcal{F}$ with $\operatorname{dim}(\mathcal{F})=d$ and a subspace $\Gamma \subset H^{0}(\mathcal{F})$.

$A$ morphism of two coherent systems $\left(\Gamma^{\prime}, \mathcal{F}^{\prime}\right) \rightarrow(\Gamma, \mathcal{F})$, is a map $f: \mathcal{F}^{\prime} \rightarrow \mathcal{F}$ such that $f_{*}\left(\Gamma^{\prime}\right) \subset \Gamma$. $\left(\Gamma^{\prime}, \mathcal{F}^{\prime}\right)$ is called coherent subsystem of $(\Gamma, \mathcal{F})$ if there is a morphism $f:\left(\Gamma^{\prime}, \mathcal{F}^{\prime}\right) \rightarrow(\Gamma, \mathcal{F})$ of coherent systems that is injective.

Definition 8.1.2 Let $\mathcal{F}$ be a coherent sheaf on $\mathbb{P}_{n}$ of codimension $d$ and let $P_{\mathbb{P}_{n}}$ be the Hilbert polynomial of $\mathcal{O}_{\mathbb{P}_{n}}$ and $P_{\mathcal{F}}$ the Hilbert polynomial of $\mathcal{F}$. We define the reduced Hilbert polynomial of a coherent system $(\Gamma, \mathcal{F})$ as follows:

$$
p_{(\Gamma, \mathcal{F})}:=\frac{\operatorname{dim}(\Gamma) P_{\mathbb{P}_{n}}+P_{\mathcal{F}}}{m(\mathcal{F})}
$$

where $m(\mathcal{F})$ is the multiplicity of $\mathcal{F}$.

Definition 8.1.3 A coherent system $(\Gamma, \mathcal{F})$ is called semistable if and only if 
a) $\mathcal{F}$ is a torsion free sheaf on its support.

b) $\forall\left(\Gamma^{\prime}, \mathcal{F}^{\prime}\right) \subset(\Gamma, \mathcal{F})$ we have

$$
p_{\left(\Gamma^{\prime}, \mathcal{F}^{\prime}\right)} \leq p_{(\Gamma, \mathcal{F})}
$$

$(\Gamma, \mathcal{F})$ is called semistable concerning $\left(\Gamma^{\prime}, \mathcal{F}^{\prime}\right)$ if $\left.\boldsymbol{b}\right)$ is satisfied for this fixed system.

As it is shown in [LP1] one can define families of coherent systems over algebraic varieties such that every fibre of such a family is a coherent system with a given Chern character ch. In particular one can define a functor Syst(ch) from the category of algebraic varieties to the category of sets for which there is a coarse moduli space Syst(ch). Due to [LP1, Th. 4.12.] Syst(ch) is a projective algebraic variety. It decomposes into the disjoint union of $\operatorname{Syst}(\mathrm{ch}, m)$, where $m=\operatorname{dim}(\Gamma)$.

Dualizing the concept of coherent systems leads to so called coherent cosystems:

Definition 8.1.4 A coherent cosystem $(\Lambda, \mathcal{E})$ of dimension $d$ on $\mathbb{P}_{n}$ consists of a coherent sheaf $\mathcal{E}$ of dimension d and a subspace $\Lambda \subset \operatorname{Ext}^{c}\left(\mathcal{E}, \mathcal{O}_{\mathbb{P}_{n}}\right)$ with $c=n-d$. $A$ morphism of coherent cosystems $(\Lambda, \mathcal{E}) \rightarrow\left(\Lambda^{\prime}, \mathcal{E}^{\prime}\right)$ of dimension d is a map $f^{*}: \operatorname{Ext}^{c}\left(\mathcal{E}^{\prime}, \mathcal{O}_{\mathbb{P}_{n}}\right) \rightarrow \operatorname{Ext}^{c}\left(\mathcal{E}, \mathcal{O}_{\mathbb{P}_{n}}\right)$ induced by a map $f: \mathcal{E} \rightarrow \mathcal{E}^{\prime}$ such that $f^{*}\left(\Lambda^{\prime}\right) \subset \Lambda$, in particular if a map $f$ induces a commutative diagram

$$
\begin{array}{ccc}
\Lambda^{\prime} & \rightarrow & \operatorname{Ext}^{c}\left(\mathcal{E}^{\prime}, \mathcal{O}_{\mathbb{P}_{n}}\right) \\
\downarrow & & \downarrow \\
\Lambda & \rightarrow & \operatorname{Ext}^{c}\left(\mathcal{E}, \mathcal{O}_{\mathbb{P}_{n}}\right)
\end{array} .
$$

Once again we define a reduced Hilbert polynomial and a stability condition for them:

Definition 8.1.5 Let $(\Lambda, \mathcal{E})$ be a coherent cosystem of dimension $d$ on $\mathbb{P}_{n}$. We set

$$
p_{(\Lambda, \mathcal{E})}:=\frac{\operatorname{dim}(\Lambda) P_{\mathbb{P}_{n}}-P_{\mathcal{E}}}{m(\mathcal{E})} .
$$

We call the cosystem semistable if

a) $\mathcal{E}$ is torsion free on its support. 
b) For any coherent cosystem $\left(\Lambda^{\prime}, \mathcal{E}^{\prime}\right) \mathcal{E}^{\prime}$ of dimension $d$ torsion free on its support such that there is a surjective $f: \mathcal{E} \rightarrow \mathcal{E}^{\prime}$ that induces a morphism of coherent cosystems $(\Lambda, \mathcal{E}) \rightarrow\left(\Lambda^{\prime}, \mathcal{E}^{\prime}\right)$ one has

$$
p_{\left(\Lambda^{\prime}, \mathcal{E}^{\prime}\right)} \leq p_{(\Lambda, \mathcal{E})} .
$$

We shall need the following results.

Lemma 8.1.6 (LP1, Lemma 5.8) A coherent cosystem $(\Lambda, \mathcal{E})$ of dimension 1 on $\mathbb{P}_{n}$ is semistable if and only if the coherent system $\left(\Lambda, \underline{E x t}^{n-1}\left(\mathcal{E}, \mathcal{O}_{\mathbb{P}_{n}}\right)\right)$ is.

Lemma 8.1.7 (LP1, Prop 4.4) Let $(\Gamma, \mathcal{F})$ be a semistable coherent system of dimension d on $\mathbb{P}_{n}$ with $\Gamma \neq 0$. Then the evaluation map

$$
\Gamma \otimes \mathcal{O}_{\mathbb{P}_{n}} \rightarrow \mathcal{F}
$$

has a cokernel of dimension $<d$.

Lemma 8.1.8 (LP1, Prop 5.3) Let $(\Gamma, \mathcal{E})$ be a semistable coherent cosystem of codimension d with $\Gamma \neq 0$. Then the cokernel of

$$
\Gamma \otimes \mathcal{O}_{\mathbb{P}_{n}} \rightarrow \underline{\operatorname{Ext}^{d}}\left(\mathcal{E}, \mathcal{O}_{\mathbb{P}_{n}}\right)
$$

is of codimension $>d$.

Furthermore in the special case of $n=2$ one can reduce the semistability of the coherent system $(\Gamma, \mathcal{F})$ to the $G$-semistability of $\mathcal{F}$ :

Lemma 8.1.9 (LP1, Lemma 6.6) Let $(\Gamma, \mathcal{F})$ be a coherent system on $\mathbb{P}_{2}$ with $\operatorname{dim}(\Gamma)=r k(\mathcal{F})$. Then $(\Gamma, \mathcal{F})$ is semistable if and only if $\mathcal{F}$ is $G$-semistable.

\subsection{Instantons and coherent systems}

Now we consider torsion free sheaves $\mathcal{F}$ on $\mathbb{P}_{2}$ with $\left(c_{1}, c_{2}\right)=(0,4)$. One has $\operatorname{ch}(\mathcal{F}(1))=2+2 h-3 h^{2}$ and $\chi(\mathcal{F})=2$. And since $h^{2}(\mathcal{F}(1))=0$ (see the proof of 4.0.6) we find that $\operatorname{Syst}\left(2+2 h-3 h^{2}\right) \neq \emptyset$. The following theorem is based on [LP1], Theorem 5.11.

Theorem 8.2.1 There is a bijection

$$
\operatorname{Syst}\left(2+2 h-3 h^{2}, 2\right) \cong \operatorname{Syst}\left(2 h+3 h^{2}, 2\right)
$$




\section{Proof:}

We construct two maps:

$$
\begin{aligned}
& \Psi: \operatorname{Syst}\left(2+2 h-3 h^{2}, 2\right) \rightarrow \operatorname{Syst}\left(2 h+3 h^{2}, 2\right) \\
& \Phi: \operatorname{Syst}\left(2 h+3 h^{2}, 2\right) \rightarrow \operatorname{Syst}\left(2+2 h-3 h^{2}, 2\right)
\end{aligned}
$$

and show $\Phi \circ \Psi=i d$ and that $\Phi$ is injective.

Construction of $\Psi$ :

Take $(\Gamma, \mathcal{F}(1)) \in \operatorname{Syst}\left(2+2 h-3 h^{3}, 2\right)$. Because of lemma 8.2.5 the evaluation map is injective. Let $\check{\theta}$ be the quotient of $\mathcal{F}(1)$ by $e v(\Gamma \otimes \mathcal{O})$ :

$$
0 \rightarrow \Gamma \otimes \mathcal{O} \rightarrow \mathcal{F}(1) \rightarrow \check{\theta} \rightarrow 0
$$

One has $\operatorname{Hom}(\mathcal{F}(1), \mathcal{O})=H^{0}\left(\mathcal{F}^{*}(-1)\right)$. By lemma 8.1.9 $\mathcal{F}$ is $G$-semistable and therefore $\mu$-semistable, so $\mathcal{F}^{*}$ is $\mu$-semistable as well, thus $H^{0}\left(\mathcal{F}^{*}(-1)\right)=0$. Therefore applying $\operatorname{Hom}(-, \mathcal{O})$ to 8.2 .1 gives

$$
0 \rightarrow \Gamma^{*} \rightarrow \operatorname{Ext}^{1}(\check{\theta}, \mathcal{O})
$$

Thus $(\Gamma, \mathcal{F}(1))$ defines a coherent cosystem $\left(\Gamma^{*}, \check{\theta}\right)$. Since $\underline{\operatorname{Hom}}(\check{\theta}, \mathcal{O})=0$ the extact sequence of low degree of the Grothendieck spectral sequence applied to $H^{0} \circ \underline{\operatorname{Hom}}(-, \mathcal{O})=\operatorname{Hom}(-, \mathcal{O})$ gives $H^{0}\left(\underline{\operatorname{Ext}} \underline{1}^{1}(\check{\theta}, \mathcal{O}) \simeq \operatorname{Ext}^{1}(\check{\theta}, \mathcal{O})\right.$. Thus $(\Gamma, \mathcal{F}(1))$ also defines a coherent system $\left(\Gamma^{*}, \theta\right)$ with $\theta:=\underline{\operatorname{Ext}^{1}}(\check{\theta}, \mathcal{O})$. We set

$$
\Psi((\Gamma, \mathcal{F}(1))):=\left(\Gamma^{*}, \theta\right)
$$

Chern character of $\theta$ :

Since $\underline{\operatorname{Ext}} \underline{x}^{2}(\check{\theta}, \mathcal{O}(-3))=0$ we have

$$
\begin{aligned}
& H^{i}(\theta(n))=H^{i}\left(\underline{\operatorname{Ext}}^{1}(\check{\theta}, \mathcal{O}(n))\right)=\operatorname{Ext}^{i+1}(\check{\theta}(-n), \mathcal{O})= \\
= & \operatorname{Ext}^{i+1}(\check{\theta}(-n-3), \mathcal{O}(-3))=H^{n-i-1}(\check{\theta}(-3) \otimes \mathcal{O}(-n)) .
\end{aligned}
$$

where the second equality again results from the Grothendieck spectral sequence applied to $H^{0} \circ \underline{\operatorname{Hom}}(-, \mathcal{O}=\operatorname{Hom}(-, \mathcal{O})$. Therefore

$$
P_{\theta}(n)=-P_{\breve{\theta}(-3)}(-n) \text {. }
$$

Thus to compute $\operatorname{ch}(\theta)$ we compute its Hilbert polynomial by computing the Hilbert polynomial of $\tilde{\theta}$, read out its Chern classes and use them to compute the 
Chern character of $\theta$. With the help of 2.6.4 we find $P_{\mathcal{F}(1)}(n)=n^{2}+5 n+2$ and $P_{\Gamma \otimes \mathcal{O}}(n)=n^{2}+3 n+2$. Therefore with the additivity of Hilbert polynomials on short exact sequences 8.2.1 leads to

$$
P_{\breve{\theta}}(n)=2 n \Rightarrow P_{\theta}(n)=-P_{\breve{\theta}(-3)}(-n)=-P_{\breve{\theta}}(-n-3)=-2(-n-3)=2 n+6 .
$$

Thus $c_{1}(\theta)=2$ and 2.6 .2 gives $\frac{3}{2} c_{1}(\theta)+\frac{1}{2}\left(c_{1}(\theta)^{2}-2 c_{2}(\theta)\right)=3+\frac{1}{2}\left(c_{1}(\theta)^{2}-2 c_{2}(\theta)\right)=$ 6 , which is equivalent to $\frac{1}{2}\left(c_{1}(\theta)^{2}-2 c_{2}(\theta)\right)=3$, giving

$$
\operatorname{ch}(\theta)=2 h+3 h^{2}
$$

Stability of $\left(\Gamma^{*}, \theta\right)$ :

Due to lemma 8.1.6 the semistability of $\left(\Gamma^{*}, \theta\right)$ is equivalent to the semistability of the coherent cosystem $\left(\Gamma^{*}, \check{\theta}\right)$. Therefore we verify the semistability of the coherent cosystem:

To see, that $\check{\theta}$ is torsion free on its support, we apply $\underline{\operatorname{Hom}}\left(-, \omega_{\mathbb{P}_{2}}\right)=$ $\underline{\operatorname{Hom}}(-, \mathcal{O}(-3))$ to 8.2.1. Since $\underline{\operatorname{Ext}} \underline{ }^{i}(\Gamma \otimes \mathcal{O}, \mathcal{O}(-3))=0$ for $i=1,2$, we find

$$
\underline{\operatorname{Ext}} t^{2}(\check{\theta}, \mathcal{O}(-3)) \cong \underline{\operatorname{Ext}}{ }^{2}(\mathcal{F}(1), \mathcal{O}(-3))
$$

Now $\mathcal{F}(1)$ is a torsion free sheaf, that is a sheaf with support of codimension 0 , torsion free on its support. By lemma 2.4.2 $\underline{E x t}^{2}(\mathcal{F}(1), \mathcal{O}(-3))=0$ and therefore $\underline{\operatorname{Ext}} t^{2}(\check{\theta}, \mathcal{O}(-3))=0$ which due to lemma 2.4 .2 means that $\check{\theta}$ is torsion free on its support.

Let $f: \check{\theta} \rightarrow \check{E} \rightarrow 0$ be given and $\check{E}$ of codimension 1 and torsion free sheaf on its support. Since $f^{*}: \operatorname{Ext}^{1}(\check{E}, \mathcal{O}) \rightarrow \operatorname{Ext}^{1}(\check{\theta}, \mathcal{O})$ is injective, $\Lambda^{*}:=\left(f^{*}\right)^{-1}\left(\Gamma^{*}\right)$ is the maximal subspace of $\Gamma^{*}$ such that $f$ induces a commutative diagram

$$
\begin{array}{ccccc}
\Lambda^{*} & \hookrightarrow & \operatorname{Ext}^{1}(\check{E}, \mathcal{O}) & =H^{0}(E) \\
\downarrow & & \downarrow f^{*} & \\
\Gamma^{*} & \hookrightarrow & \operatorname{Ext}^{1}(\check{\theta}, \mathcal{O}) & =H^{0}(\theta) .
\end{array}
$$

Let $i$ be the element of $E x t^{1}(\check{E}, \Lambda \otimes \mathcal{O})$ that corresponds to the upper inclusion and $\tilde{i}$ the element of $\operatorname{Ext}^{1}(\tilde{\theta}, \Gamma \otimes \mathcal{O})$ that corresponds to the lower inclusion. Let $\check{K}$ be the kernel of $f$ and $C^{*}$ the cokernel of $\Lambda^{*} \hookrightarrow \Gamma^{*}$. We can form a 
cross out of the two sequences that result from applying $\operatorname{Hom}(-, \Lambda \otimes \mathcal{O})$ to $0 \rightarrow \check{K} \rightarrow \check{\theta} \rightarrow \check{E} \rightarrow 0$ and $\operatorname{Hom}(\check{\theta},-)$ to $0 \rightarrow C \otimes \mathcal{O} \rightarrow \Gamma \otimes \mathcal{O} \rightarrow \Lambda \otimes \mathcal{O} \rightarrow 0$ :

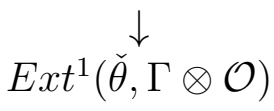

$$
\begin{aligned}
& 0 \rightarrow \operatorname{Ext}^{1}(\check{E}, \Lambda \otimes \mathcal{O}) \stackrel{\beta}{\rightarrow} \operatorname{Ext}^{1}(\check{\theta}, \Lambda \otimes \mathcal{O}) \rightarrow . \\
& \downarrow \\
& 0
\end{aligned}
$$

The commutativity of 8.2.2 means that $\alpha(\tilde{i})=\beta(i)$. Since $\tilde{i}$ corresponds to the extension 8.2.1, we get a map from 8.2.1 to an element of $\operatorname{Ext}^{1}(\tilde{E}, \Lambda \otimes \mathcal{O})$, in particular a commutative diagram

$$
\begin{aligned}
& 0 \rightarrow \Gamma \otimes \mathcal{O} \rightarrow \mathcal{F}(1) \rightarrow \check{\theta} \rightarrow 0 \\
& 0 \rightarrow \Lambda \otimes \stackrel{\downarrow}{\otimes} \rightarrow \stackrel{\downarrow}{\mathcal{J}} \rightarrow \stackrel{\downarrow}{\check{E}} \rightarrow 0
\end{aligned}
$$

where the right vertical arrow is $f$ and therefore surjective and so is the left vertical arrow, which is just restriction. Thus the middle vertical arrow is a surjection as well. Now we can use the $G$-semistability of $\mathcal{F}(1)$, which is

$$
P_{\mathcal{J}} \geq \frac{r k(\mathcal{J})}{r k(\mathcal{F})} P_{\mathcal{F}(1)} .
$$

By the additivity of the Hilbert polynomial on short exact sequences, since $P_{\mathcal{O}}(n)=\frac{1}{2} n^{2}+\frac{3}{2} n+1$, we get from 8.2 .3

$$
\begin{aligned}
& P_{\mathcal{J}}(n)=P_{\check{E}}(n)+r k(\mathcal{J})\left(\frac{1}{2} n^{2}+\frac{3}{2} n+1\right) \\
& P_{\mathcal{F}}(n)=P_{\check{\theta}}(n)+r k(\mathcal{F})\left(\frac{1}{2} n^{2}+\frac{3}{2} n+1\right)
\end{aligned}
$$

Plugging this into 8.2.4 leads to

$$
\begin{aligned}
& P_{\breve{E}}(n)+r k(\mathcal{J})\left(\frac{1}{2} n^{2}+\frac{3}{2} n+1\right) \geq \frac{r k(\mathcal{J})}{r k(\mathcal{F})}\left(P_{\ddot{\theta}}(n)+r k(\mathcal{F})\left(\frac{1}{2} n^{2}+\frac{3}{2} n+1\right)\right)= \\
= & \frac{r k(\mathcal{J})}{r k(\mathcal{F})} P_{\breve{\theta}}(n)+r k(\mathcal{J})\left(\frac{1}{2} n^{2}+\frac{3}{2} n+1\right)
\end{aligned}
$$

Thus 


$$
P_{\check{E}} \geq \frac{r k(\mathcal{J})}{r k(\mathcal{F})} P_{\check{\theta}}
$$

Now since $\check{\theta}$ and $\check{E}$ are both of codimension 1, their multiplicity is the leading coefficient in their Hilbert polynomial and therefore this inequality implies

$$
\frac{m(\check{E})}{m(\check{\theta})} \geq \frac{r k(\mathcal{J})}{r k(\mathcal{F})}=\frac{\operatorname{dim}(\Lambda)}{\operatorname{dim}(\Gamma)}=\frac{\operatorname{dim}\left(\Lambda^{*}\right)}{\operatorname{dim}\left(\Gamma^{*}\right)}
$$

where the first ' $=$ ' results from the injectivity of the evaluation maps in 8.2.3. Thus

$$
\frac{\operatorname{dim}\left(\Lambda^{*}\right)}{m(\check{E})} \leq \frac{\operatorname{dim}\left(\Gamma^{*}\right)}{m(\check{\theta})}
$$

which in case of inequality means that $\left(\Gamma^{*}, \check{\theta}\right)$ is stable concerning $\left(\Lambda^{*}, \check{E}\right)$. In case of equality we have $\frac{m(\check{E})}{m(\dot{\theta})}=\frac{r k(\mathcal{J})}{r k(\mathcal{F})}$ and therefore 8.2 .5 becomes

$$
P_{\check{E}} \geq \frac{m(\check{E})}{m(\check{\theta})} P_{\check{\theta}}
$$

which means that $\left(\Gamma^{*}, \check{\theta}\right)$ is semistable concerning $\left(\Lambda^{*}, \check{E}\right)$. Thus the semistability is proved.

Construction of $\Phi$ :

The constuction of this map is simple: Since a coherent system $\left(\Gamma^{*}, \theta\right)$ corresponds to a coherent cosystem $\left(\Gamma^{*}, \check{\theta}\right)$, and $\left(\Gamma^{*} \hookrightarrow \operatorname{Ext}^{1}(\check{\theta}, \mathcal{O})\right) \in \operatorname{Ext}^{1}(\check{\theta}, \Gamma \otimes \mathcal{O})$, a coherent system $\left(\Gamma^{*}, \theta\right)$ induces an extension

$$
0 \rightarrow \Gamma \otimes \mathcal{O} \rightarrow \mathcal{F}(1) \rightarrow \check{\theta} \rightarrow 0
$$

We define

$$
\Phi\left(\Gamma^{*}, \theta\right):=(\Gamma, \mathcal{F}(1))
$$

The computation of the Chern character from above works in the other direction as well, thus we find that the Chern character of $\mathcal{F}(1)$ is as stated. 
Stability of $(\Gamma, \mathcal{F}(1))$ :

By dualizing 8.2.6 we find

$$
0 \rightarrow \mathcal{F}^{*} \rightarrow \Gamma^{*} \otimes \mathcal{O} \rightarrow \underline{\operatorname{Ext}^{1}}(\check{\theta}, \mathcal{O}) \rightarrow \underline{\operatorname{Ext}}^{1}(\mathcal{F}(1), \mathcal{O}) \rightarrow 0
$$

and $\underline{\operatorname{Ext}}^{2}(\check{\theta}, \mathcal{O}(-3)) \cong \underline{E x t}^{2}(\mathcal{F}(1), \mathcal{O}(-3))$. $\check{\theta}$ is torsion free on its support, therefore $\operatorname{Ext}^{2}(\mathcal{F}(1), \mathcal{O}(-3))=0$, furthermore by lemma 8.1.8 the codimension of $\operatorname{supp}\left(\underline{\operatorname{Ext}^{1}}(\mathcal{F}(1), \mathcal{O}(-3))\right)$ is $\geq 2$. Thus by lemma 2.4.2 $\mathcal{F}(1)$ is torsion free.

Now let $\left(\Gamma^{\prime}, \mathcal{F}^{\prime}\right) \subset(\Gamma, \mathcal{F}(1))$. That means, we have a commutative diagram

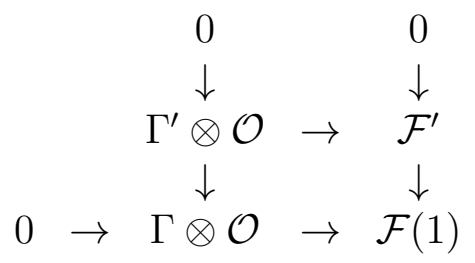

Because of the injectivity of the lower horizontal and the two vertical arrows, the upper horizontal is injective as well, and we can extend the diagram to

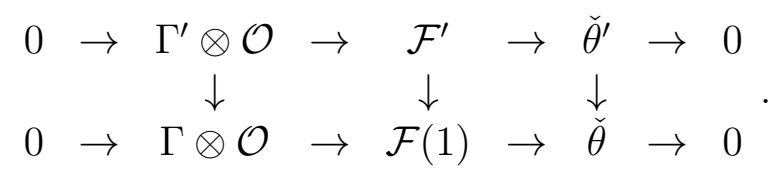

The first two vertical arrows are injective, therefore so is the third, thus we have constructed an injective morphism $0 \rightarrow \check{\theta}^{\prime} \rightarrow \check{\theta}$.

Since for a sheaf of rank $>0$ on $\mathbb{P}_{2}$ the rank is the same as the multiplicity, it follows from diagram 8.2.7 that

$$
\frac{\operatorname{dim}\left(\Gamma^{\prime}\right)}{m\left(\mathcal{F}^{\prime}\right)}=\frac{\operatorname{dim}(\Gamma)}{m(\mathcal{F}(1))}=1
$$

Therefore the semistability of $(\Gamma, \mathcal{F}(1))$ concerning $\left(\Gamma^{\prime}, \mathcal{F}^{\prime}\right)$ is equivalent to $p_{\mathcal{F}^{\prime}} \leq$ $p_{\mathcal{F}(1)}$. If $\operatorname{dim}\left(\Gamma^{\prime}\right)=r k\left(\mathcal{F}^{\prime}\right)=2$ this is true, since in that case $p_{\mathcal{F}^{\prime}} \leq p_{\mathcal{F}(1)}$ is equivalent to $P_{\mathcal{F}^{\prime}} \leq P_{\mathcal{F}(1)}$, the map $\mathcal{F}^{\prime} \rightarrow \mathcal{F}(1)$ is an injection and the Hilbert polynomial is additive on short exact sequences. Thus we only have to consider the case of $\operatorname{dim}\left(\Gamma^{\prime}\right)=r k\left(\mathcal{F}^{\prime}\right)=1$. Now we use the semistability of the coherent cosystem: Since we have an injection $0 \rightarrow \check{\theta}^{\prime} \rightarrow \check{\theta}$, the semistability of $\left(\Gamma^{*}, \check{\theta}\right)$ implies 


$$
\frac{\operatorname{dim}\left(\Gamma^{\prime}\right)}{m\left(\check{\theta}^{\prime}\right)} \geq \frac{\operatorname{dim}(\Gamma)}{m(\check{\theta})}
$$

The right hand side is 1 . For that inequality to hold, we need $m\left(\check{\theta}^{\prime}\right)=1$, which means that ?? is an equality, thus the semistability of $\left(\Gamma^{*}, \check{\theta}\right)$ concerning $\left(\left(\Gamma^{\prime}\right)^{*}, \check{\theta}^{\prime}\right)$ induces $p_{\check{\theta}^{\prime}} \leq p_{\check{\theta}}$. That is

$p_{\check{\theta}^{\prime}}=\frac{P_{\check{\theta}^{\prime}}}{m\left(\check{\theta}^{\prime}\right)}=\frac{P_{\check{\theta}^{\prime}}}{\operatorname{dim}\left(\Gamma^{\prime}\right)}=\frac{P_{\mathcal{F}^{\prime}}-P_{\Gamma^{\prime} \otimes \mathcal{O}}}{\operatorname{dim}\left(\Gamma^{\prime}\right)}=p_{\mathcal{F}^{\prime}}-P_{\mathcal{O}} \leq p_{\check{\theta}}=\frac{P_{\mathcal{F}(1)}-P_{\Gamma \otimes \mathcal{O}}}{\operatorname{dim}(\Gamma)}=p_{\mathcal{F}(1)}-P_{\mathcal{O}}$

Thus

$$
p_{\mathcal{F}^{\prime}} \leq p_{\mathcal{F}(1)}
$$

and the semistability of $(\Gamma, \mathcal{F}(1))$ concerning $\left(\Gamma^{\prime}, \mathcal{F}^{\prime}\right)$ is shown. Thus $(\Gamma, \mathcal{F}(1))$ is semistable.

$\Phi \circ \Psi=i d:$

We have a 1-to-1 correspondence between coherent systems $(\Gamma, \mathcal{F}(1))$ and short exact sequences

$$
0 \rightarrow \Gamma \otimes \mathcal{O} \rightarrow \mathcal{F}(1) \rightarrow \check{\theta} \rightarrow 0
$$

The image of $(\Gamma, \mathcal{F}(1))$ under $\Psi$ is the coherent system $\left(\Gamma^{*}, \theta\right)$, which uniquely determines an inclusion $\Gamma^{*} \hookrightarrow \operatorname{Ext}^{1}(\check{\theta}, \mathcal{O})$, which corresponds to an element of $\operatorname{Ext}^{1}(\check{\theta}, \Gamma \otimes \mathcal{O})$. The image of $\left(\Gamma^{*}, \theta\right)$ under $\Phi$ is defined via this extension, thus to show $\Phi \circ \Psi=i d$, we have to show, that the extension that corresponds to $\Gamma^{*} \hookrightarrow \operatorname{Ext}^{1}(\check{\theta}, \mathcal{O})$ is 8.2.9.

If we apply $\operatorname{Hom}(-, \Gamma \otimes \mathcal{O})$ to 8.2 .9 , we get

$$
0 \rightarrow \Gamma^{*} \otimes \Gamma \rightarrow \operatorname{Ext}^{1}(\check{\theta}, \Gamma \otimes \mathcal{O}) \rightarrow \operatorname{Ext}^{1}(\mathcal{F}(1), \Gamma \otimes \mathcal{O}) \rightarrow 0
$$

It is known, that the image of the identity of $\Gamma^{*} \otimes \Gamma$ under this construction is the extension that one has started with. But the image of the identity in $\operatorname{Ext}^{1}(\check{\theta}, \Gamma \otimes \mathcal{O})=\operatorname{Ext}^{1}(\check{\theta}, \mathcal{O}) \otimes \Gamma$ is the inclusion $\Gamma^{*} \hookrightarrow \operatorname{Ext}^{1}(\check{\theta}, \mathcal{O})$. Therefore the extension that corresponds to $\Gamma^{*} \hookrightarrow \operatorname{Ext}(\check{\theta}, \mathcal{O})$ is 8.2.9.

$\Phi$ is injective: 
Assume that $\Phi\left(\left(\left(\Gamma^{\prime}\right)^{*}, \theta^{\prime}\right)\right)=\Phi\left(\left(\Gamma^{*}, \theta\right)\right)$. First of all that would imply $\check{\theta}^{\prime}=\check{\theta}$ and therefore $\theta^{\prime}=\theta$. Thus if $\left(\left(\Gamma^{\prime}\right)^{*}, \theta^{\prime}\right) \neq\left(\Gamma^{*}, \theta\right)$ they represent two different extensions of $\check{\theta}$ by $V \otimes \mathcal{O}$, where $V$ is a 2 dimensional vector space, as $E x t^{1}$ classifies the set of extensions up to an isomorphism. Thus the resulting sheaves $\mathcal{F}(1)$ and $\mathcal{F}^{\prime}(1)$ cannot be the identical.

That completes the proof.

In the proof of lemma 4.0.6 we have seen that $h^{2}(\mathcal{F}(1))=0$, furthermore in [LP1] it is shown that $h^{1}(\mathcal{F}) \leq 1$ and since $\chi(\mathcal{F}(1))=h^{0}(\mathcal{F}(1))-h^{1}(\mathcal{F}(1))=2$ one has $2 \leq h^{0}(\mathcal{F}(1)) \leq 3$.

The sheaves with $h^{1}(\mathcal{F}(1))=1$, so called special sheaves, are locally free, form a set of codimension 3 and have a unique jumping line of order 3 . In fact Syst $\left(2+2 h-3 h^{3}, 2\right)$ is a blowup of $\mathfrak{M}_{\text {Gss }}(4)$ along the set of special sheaves. (See $[\mathrm{LP} 1])$

For $(\Gamma, \theta) \in \operatorname{Syst}\left(2+2 h-3 h^{3}, 2\right)$ we denote the map, that evaluates a section $s \in \Gamma$ in $\theta$ by $\overline{e v}: \Gamma \otimes \mathcal{O} \rightarrow \theta$. We apply $\underline{\operatorname{Hom}}(-, \mathcal{O})$ to the standard sequence of the coherent system 8.2.1:

$$
0 \rightarrow \mathcal{F}^{*}(-1) \rightarrow \Gamma \otimes \mathcal{O} \stackrel{\overline{e v}}{\rightarrow} \theta \rightarrow \underline{\operatorname{Ext}^{1}}(\mathcal{F}(1), \mathcal{O}) \rightarrow 0
$$

$\underline{\operatorname{Ext}} t^{1}(\mathcal{F}(1), \mathcal{O})$ is the obstruction for $\mathcal{F}$ to be locally free, thus we found the following lemma:

Lemma 8.2.2 Let $(\Gamma, \theta) \in \operatorname{Syst}\left(2 h+3 h^{2}, 2\right)$ and $\Phi((\Gamma, \theta))=\left(\Gamma^{*}, \mathcal{F}(1)\right)$. Then $\mathcal{F}$ is locally free if and only if the evaluation map

$$
\overline{e v}: \Gamma \otimes \mathcal{O} \rightarrow \theta
$$

is surjective.

Definition 8.2.3 Let $(\Gamma, \theta) \in \operatorname{Syst}\left(2 h+3 h^{2}, 2\right)$. A point $p \in \mathbb{P}_{2}$ such that the restriction of $\overline{e v}$ to the stalks at $p$ is not surjective, is called base point of the coherent system. If $(\Gamma, \theta)$ has no base point, we call it base point free.

Corollary 8.2.4 In the above setup $p$ is a base point if and only if $p \in$ $\operatorname{setsupp}\left(\underline{\operatorname{Ext}^{1}}(\mathcal{F}(1), \mathcal{O})\right)$.

Lemma 8.2.5 A coherent system $(\Gamma, \theta) \in \operatorname{Syst}\left(2 h+3 h^{2}, 2\right)$ has a base point if and only if there is $a \vartheta \subset \theta$ such that $\Gamma \subset H^{0}(\vartheta)$. 


\section{Proof:}

If there is a $\vartheta \subset \theta$ such that $\Gamma \subset H^{0}(\vartheta)$, then $\overline{e v}(\Gamma \otimes \mathcal{O}) \subset \vartheta$, since $\Gamma \otimes \mathcal{O}$ is globally generated.

Suppose $(\Gamma, \theta)$ has a base point, that is $\underline{\operatorname{Ext}} t^{1}(\mathcal{F}(1), \mathcal{O}) \neq 0$. Thus there is a subsheaf $\vartheta \subset \theta$, such that $\underline{\operatorname{Ext}}(\mathcal{F}(1), \mathcal{O})$ is the quotient of $\theta$ by $\vartheta$ and we have

$$
0 \rightarrow \mathcal{F}^{*}(-1) \rightarrow \Gamma \otimes \mathcal{O} \rightarrow \vartheta \rightarrow 0 .
$$

Since $\mathcal{F}$ is torsion free and stable its dual is stable as well and has the same first Chern class, therefore $H^{0}\left(\mathcal{F}^{*}(-1)\right)=0$ and the long exact sequence of cohomology gives

$$
0 \rightarrow \Gamma \rightarrow H^{0}(\vartheta)
$$

This completes the proof.

\subsection{Spectral schemes and coherent systems}

In chapter 6.2 we found that the spectral scheme of an instanton can be described in terms of the associated $G$-semistable sheaf $\mathcal{F}$, in particular

$$
\Sigma_{\mathcal{F}}=\operatorname{Fitt}_{0}\left(\underline{\operatorname{Exp}}_{p}^{1}\left(q^{*}(\mathcal{F}(1)), \mathcal{O}_{D}\right)\right)
$$

We computed the Fitting scheme by using the resolution

$$
0 \rightarrow H^{1}(\mathcal{F}(-2)) \otimes \mathcal{O}_{\mathbb{P}_{2}^{*}}(-1) \rightarrow H^{1}(\mathcal{F}(-1)) \otimes \mathcal{O}_{\mathbb{P}_{2}^{*}} \rightarrow \underline{\operatorname{Exp}}_{p}^{1}\left(q^{*}(\mathcal{F}(1)), \mathcal{O}_{D}\right) \rightarrow 0
$$

Now we will show that there is a second resolution of $\underline{\operatorname{Exp}}_{p}^{1}\left(q^{*}(\mathcal{F}(1)), \mathcal{O}_{D}\right)$ only using sheaves, that are related to a corresponding coherent system $(\Gamma, \theta)$. For a non special sheaf $\mathcal{F}(1)$ this system is unique but even for a special $\mathcal{F}(1)$ we get for any $\Phi\left(\Gamma^{*}, \mathcal{F}(1)\right)$ with $\Gamma^{*} \subset H^{0}(\mathcal{F}(1))$ a resolution of $\operatorname{Exp}_{p}^{1}\left(q^{*}(\mathcal{F}(1)), \mathcal{O}_{D}\right)$. Therefore the notations of spectral scheme and spectral curve of a coherent system, which we denote by $\Sigma_{(\Gamma, \theta)}$ respectively $\tilde{\Sigma}_{(\Gamma, \theta)}$, are well defined.

Consider $\overline{e v}: \Gamma \otimes \mathcal{O} \rightarrow \theta$. When we apply $p_{*}\left(q^{*}(-) \otimes \mathcal{O}_{D}\right)$ we get a map

$$
e v: \Gamma \otimes \mathcal{O}_{\mathbb{P}_{2}^{*}} \rightarrow \mathcal{G}_{\theta}:=p_{*}\left(q^{*}(\theta) \otimes \mathcal{O}_{D}\right)
$$

Note that if $S_{\theta}:=\left\{\check{l} \in \mathbb{P}_{2}^{*}: l \subset \operatorname{supp}(\theta)\right\}$ then $\mathcal{G}_{\theta}$ is locally free of on $\mathbb{P}_{2}^{*} \backslash S_{\theta}$ by the base-change-theorem and that the induced map of fibres $e v_{\breve{l}}$ maps a section $s \in \Gamma$ to $\left.s\right|_{l} \in H^{0}\left(\left.\theta\right|_{l}\right)=\left(\mathcal{G}_{\theta}\right)_{\grave{l}}$ 
Theorem 8.3.1 ([LPQ $]$ )

Let $(\Gamma, \theta) \in \operatorname{Syst}\left(2 h+3 h^{2}, 2\right)$ and $\Phi(\Gamma, \theta)=\left(\Gamma^{*}, \mathcal{F}(1)\right)$. The evaluation map ev is injective. The Fitting scheme of its cokernel is $\Sigma_{\mathcal{F}}$.

$$
0 \rightarrow \Gamma \otimes \mathcal{O}_{\mathbb{P}_{2}^{*}} \rightarrow \mathcal{G}_{\theta} \rightarrow \underline{\operatorname{Ext}}_{p}^{1}\left(q^{*}(\mathcal{F}(1)), \mathcal{O}_{D}\right) \rightarrow 0
$$

\section{Proof:}

Step 1: We show that $\mathcal{G}_{\theta} \cong \operatorname{Ext}_{p}^{1}\left(q^{*}(\theta), \mathcal{O}_{D}\right)$.

Consider the resolution of $\mathcal{O}_{D}$. Since the supports of $q^{*}(\theta)$ and $\mathcal{O}_{D}$ are transversal, we have $\underline{\operatorname{Tor}^{1}}\left(q^{*}(\theta), \mathcal{O}_{D}\right)=0$, thus after tensoring with $q^{*}(\theta)$ the resolution remains short exact:

$$
0 \rightarrow q^{*}(\theta)(-1,-1) \stackrel{\cdot s_{D}}{\rightarrow} q^{*}(\theta) \rightarrow q^{*}(\theta) \otimes \mathcal{O}_{D} \rightarrow 0
$$

We apply $p_{*}$ :

$$
0 \rightarrow H^{0}(\theta(-1)) \otimes \mathcal{O}(-1) \rightarrow H^{0}(\theta) \otimes \mathcal{O} \rightarrow \mathcal{G}_{\theta} \rightarrow H^{1}(\theta(-1)) \otimes \mathcal{O}(-1)
$$

We show, that $H^{1}(\theta(-1)) \otimes \mathcal{O}(-1)=0$. Consider

$$
0 \rightarrow \Gamma \otimes \mathcal{O}(-i-3) \rightarrow \mathcal{F}(1-i-3) \rightarrow \check{\theta}(-i-3) \rightarrow 0
$$

Applying the cohomology functor gives

$$
H^{0}(\mathcal{F}(1-i-3)) \rightarrow H^{0}(\check{\theta}(-i-3)) \rightarrow \Gamma \otimes H^{1}(\mathcal{O}(-i-3))
$$

Since we are on $\mathbb{P}_{2}, H^{1}(\mathcal{O}(-i-3))=0$. Due to the $\mu$-semistability of $\mathcal{F}$ we have $H^{0}(\mathcal{F}(1-i-3))=0$ for $1-i-3 \leq-1$, which is $i \geq-1$. Thus

$$
H^{0}(\check{\theta}(-i-3))=0 \text { for } i \geq-1
$$

Now Serre duality gives

$$
H^{1}(\theta(i))^{*} \cong \operatorname{Ext}^{1}(\theta(i), \mathcal{O}(-3))=H^{0}\left(\underline{\operatorname{Ext}^{1}}(\theta(i), \mathcal{O}(-3))=H^{0}(\check{\theta}(-i-3))\right.
$$


Thus

$$
H^{1}(\theta(i))=0 \text { for } i \geq-1
$$

and we have found a resolution of $\mathcal{G}_{\theta}$

$$
0 \rightarrow H^{0}(\theta(-1)) \otimes \mathcal{O}(-1) \stackrel{\cdot\left(s_{D}\right)_{*}}{\rightarrow} H^{0}(\theta) \otimes \mathcal{O} \rightarrow \mathcal{G}_{\theta} \rightarrow 0
$$

Now we show, that $\underline{\operatorname{Ext}}_{p}^{1}\left(q^{*}(\check{\theta}), \mathcal{O}_{D}\right)$ has the same resolution and is therefore isomorphic to $\mathcal{G}_{\theta}$. To show that, we will need the natural isomorphism

$$
\underline{\operatorname{Ext}^{i}}\left(q^{*}(\check{\theta}), \mathcal{O}\right) \simeq q^{*}\left(\underline{\operatorname{Ext}} t^{i}(\check{\theta}, \mathcal{O})\right)
$$

To see this consider the two functors $\underline{\operatorname{Hom}}\left(-, \mathcal{O}_{\mathbb{P}_{2} \times \mathbb{P}_{2}^{*}}\right) \circ q^{*}$ and $q^{*} \circ \underline{\operatorname{Hom}}\left(-, \mathcal{O}_{\mathbb{P}_{2}}\right)$. By their very definition they are the same and since $q^{*}$ is exact, the corresponding Grothendieck spectral sequences have only nonzero entries in the first column and row respectively. Therefore we find 8.3.5.

Since $\check{\theta}$ is torsion free on its support one has

$$
\begin{gathered}
R^{2} p_{*}\left(\underline{\operatorname{Hom}}\left(q^{*}(\check{\theta}), \mathcal{O}(-1,-1)\right)=0,\right. \\
R^{1} p_{*}\left(\underline{E x t^{1}}\left(q^{*}(\check{\theta}), \mathcal{O}(-1,-1)\right)=R^{1} p_{*}\left(q *\left(\underline{E x t^{1}}(\check{\theta}, \mathcal{O}) \otimes \mathcal{O}(-1,-1)\right)\right)=\right. \\
\left.=\quad H^{1}(\theta(-1)) \otimes \mathcal{O}(-1)\right) \quad=0 \\
p_{*}\left(\underline{E x t^{2}}\left(q^{*}(\check{\theta}), \mathcal{O}(-1,-1)\right)=p_{*}\left(q^{*}\left(\underline{\underline{E x t}}{ }^{2}(\check{\theta}, \mathcal{O}) \otimes \mathcal{O}(-1,-1)\right)\right)=0,\right.
\end{gathered}
$$

with the help of the Grothendieck spectral sequence $\underline{\operatorname{Ext}}_{p}^{2}\left(q^{*}(\check{\theta}), \mathcal{O}(-1,-1)\right)=$ 0 , therefore applying $\underline{\operatorname{Hom}}_{p}\left(q^{*}(\check{\theta}),-\right)$ to the resolution of $\mathcal{O}_{D}$ gives with $\underline{\operatorname{Hom}}_{p}\left(q^{*}(\check{\theta}), \mathcal{O}_{D}\right)$
$\left(s_{D}\right)_{*}$
$0 \rightarrow \underline{\operatorname{Ext}}_{p}^{1}\left(q^{*}(\check{\theta}), \mathcal{O}(-1,-1)\right) \quad \rightarrow \quad \underline{\operatorname{Ext}}_{p}^{1}\left(q^{*}(\check{\theta}), \mathcal{O}\right) \rightarrow \underline{\operatorname{Ext}}_{p}^{1}\left(q^{*}(\check{\theta}), \mathcal{O}_{D}\right) \rightarrow 0$ 
Furthermore because of the vanishing of $\underline{\operatorname{Hom}}\left(q^{*}(\check{\theta}), \mathcal{O}(-1,-1)\right)$ and $\underline{\operatorname{Hom}}\left(q^{*}(\check{\theta}), \mathcal{O}\right)$ we have natural isomorphisms

$$
\begin{aligned}
\underline{\operatorname{Ext}}_{p}^{1}\left(q^{*}(\check{\theta}), \mathcal{O}(-1,-1)\right) & \simeq p_{*} \underline{\operatorname{Ext}}^{1}\left(q^{*}(\check{\theta}), \mathcal{O}(-1,-1)\right), \\
\underline{\operatorname{Ext}}_{p}^{1}\left(q^{*}(\check{\theta}), \mathcal{O}\right) & \simeq p_{*} \underline{\operatorname{Ext}}^{1}\left(q^{*}(\check{\theta}), \mathcal{O}\right)
\end{aligned}
$$

and get therefore

$$
0 \rightarrow p_{*} \underline{\operatorname{Ext}^{1}}\left(q^{*}(\check{\theta}), \mathcal{O}(-1,-1)\right) \stackrel{\left(s_{D}\right)_{*}}{\rightarrow} p_{*} \underline{\operatorname{Ext}}^{1}\left(q^{*}(\check{\theta}), \mathcal{O}\right) \rightarrow \underline{\operatorname{Ext}}_{p}^{1}\left(q^{*}(\check{\theta}), \mathcal{O}_{D}\right) \rightarrow 0
$$

which becomes with 8.3.5 and the isomorphism $\underline{\operatorname{Ext}}{ }^{1}\left(q^{*}(\check{\theta}), \mathcal{O}(-1,-1)\right) \simeq$ $\underline{\operatorname{Ext}^{1}}\left(q^{*}(\check{\theta}), \mathcal{O}\right) \otimes \mathcal{O}(-1,-1)$

$$
p_{*}\left(q^{*}(\theta) \otimes \mathcal{O}(-1,-1)\right) \stackrel{\left(s_{D}\right)_{*}}{\rightarrow} p_{*} q^{*}(\theta) \rightarrow \underline{\operatorname{Ext}}_{p}^{1}\left(q^{*}(\check{\theta}), \mathcal{O}_{D}\right) \rightarrow 0 .
$$

Therefore

$$
\mathcal{G}_{\theta} \cong \underline{\operatorname{Ext}}_{p}^{1}\left(q^{*}(\check{\theta}), \mathcal{O}_{D}\right)
$$

\section{Step 2:}

The resolution of $\underline{\operatorname{Ext}}_{p}\left(q^{*}(\mathcal{F}(1)), \mathcal{O}_{D}\right)$ :

We apply $\underline{\operatorname{Hom}}_{p}\left(-, \mathcal{O}_{D}\right)$ to $0 \rightarrow \Gamma^{*} \otimes \mathcal{O} \rightarrow q^{*}(\mathcal{F}(1)) \rightarrow q^{*}(\check{\theta}) \rightarrow 0$ :

$$
\begin{aligned}
& 0 \rightarrow p_{*} \underline{\operatorname{Hom}}\left(q^{*}(\mathcal{F}(1)), \mathcal{O}_{D}\right) \rightarrow \Gamma \otimes \mathcal{O} \quad \rightarrow \\
& \rightarrow \quad \underline{\operatorname{Ext}}_{p}^{1}\left(q^{*}(\check{\theta}), \mathcal{O}_{D}\right) \rightarrow \underline{\operatorname{Ext}}_{p}^{1}\left(q^{*}(\mathcal{F}(1)), \mathcal{O}_{D}\right) \rightarrow 0 \\
& \text { ? } \\
& \mathcal{G}_{\theta}
\end{aligned}
$$

$\Gamma \otimes \mathcal{O}$ and $\mathcal{G}_{\theta}$ are torsion free sheaves of rank 2 and the cokernel of the third arrow of rank 0 , therefore $p_{*} \operatorname{Hom}\left(q^{*}(\mathcal{F}(1)), \mathcal{O}_{D}\right)$ is also of rank 0 , which means that is must be 0 , since otherwise it would be a torsion subsheaf of $\Gamma \otimes \mathcal{O}$. Thus we have our resolution

$$
0 \rightarrow \Gamma \otimes \mathcal{O} \stackrel{\gamma}{\rightarrow} \mathcal{G} \rightarrow \underline{\operatorname{Ext}}_{p}^{1}\left(q^{*}(\mathcal{F}(1)), \mathcal{O}_{D}\right) \rightarrow 0
$$




\section{Step 3:}

We show that $\gamma=e v$. If $\mathcal{F}$ is locally free, $\underline{\operatorname{Ext}} t^{1}\left(q^{*}(\mathcal{F}(1)), \mathcal{O}_{D}\right)=$ $\underline{\operatorname{Ext}} t^{1}\left(\mathcal{O}, \mathcal{O}_{D}\right) \otimes\left(q^{*}(\mathcal{F}(1))\right)^{*}=0$, since $\underline{\operatorname{Ext}^{1}}\left(\mathcal{O}, \mathcal{O}_{D}\right)=0$ (which one gets by applying $\underline{\operatorname{Hom}}(\mathcal{O},-)$ to the resolution of $\left.\mathcal{O}_{D}\right)$. Therefore $\underline{\operatorname{Ext}}_{p}^{1}\left(q^{*}(F(1)), \mathcal{O}_{D}\right) \simeq$ $R^{1} p_{*}\left(\underline{\operatorname{Hom}}\left(q^{*}(\mathcal{F}(1)), \mathcal{O}_{D}\right)\right)$ and 8.3.6 becomes

$$
0 \rightarrow \Gamma \otimes \mathcal{O} \rightarrow \mathcal{G} \rightarrow R^{1} p_{*}\left(\underline{\operatorname{Hom}}\left(q^{*}(\mathcal{F}(1)), \mathcal{O}_{D}\right)\right) \rightarrow 0
$$

In this locally free case there is a second way to construct 8.3.6 out of the standard sequence of the coherent system: Since $\underline{\operatorname{Ext}}{ }^{1}(\mathcal{F}(1), \mathcal{O})=0$ the dual sequence of $0 \rightarrow \Gamma^{*} \otimes \mathcal{O} \rightarrow \mathcal{F}(1) \rightarrow \check{\theta} \rightarrow 0$ is still exact:

$$
0 \rightarrow \mathcal{F}(-1) \rightarrow \Gamma \otimes \mathcal{O} \rightarrow \theta \rightarrow 0
$$

Here the third arrow is the evaluation of a section of $\Gamma$ in $\theta$. Then we apply $q^{*}$, tensor with $\mathcal{O}_{D}$ and apply $p_{*}$ to get 8.3.6. Here one can directly see that $\gamma=e v$. If $\mathcal{F}$ is not locally free, consider $\vartheta=\operatorname{ker}\left(\theta \rightarrow \underline{\operatorname{Ext}^{1}}(\mathcal{F}(1), \mathcal{O})\right)$ and

$$
0 \rightarrow \mathcal{F}^{*}(-1) \rightarrow \Gamma \otimes \mathcal{O} \rightarrow \vartheta \rightarrow 0
$$

Applying $p_{*}\left(q^{*}(-) \otimes \mathcal{O}_{D}\right)$ we get

$$
e v_{\vartheta}: \Gamma \otimes \mathcal{O} \rightarrow \mathcal{G}_{\vartheta}
$$

Furthermore we have the following diagram (we set $E_{\mathcal{F}}=\underline{\operatorname{Ext}} t^{1}(\mathcal{F}(1), \mathcal{O})$ ):

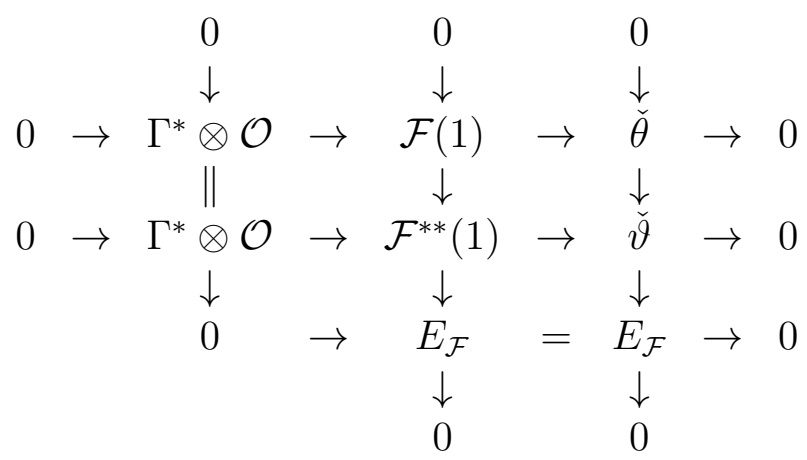

Now we apply $\underline{\operatorname{Hom}}_{p}\left(q^{*}(-), \mathcal{O}_{D}\right)$. We get 


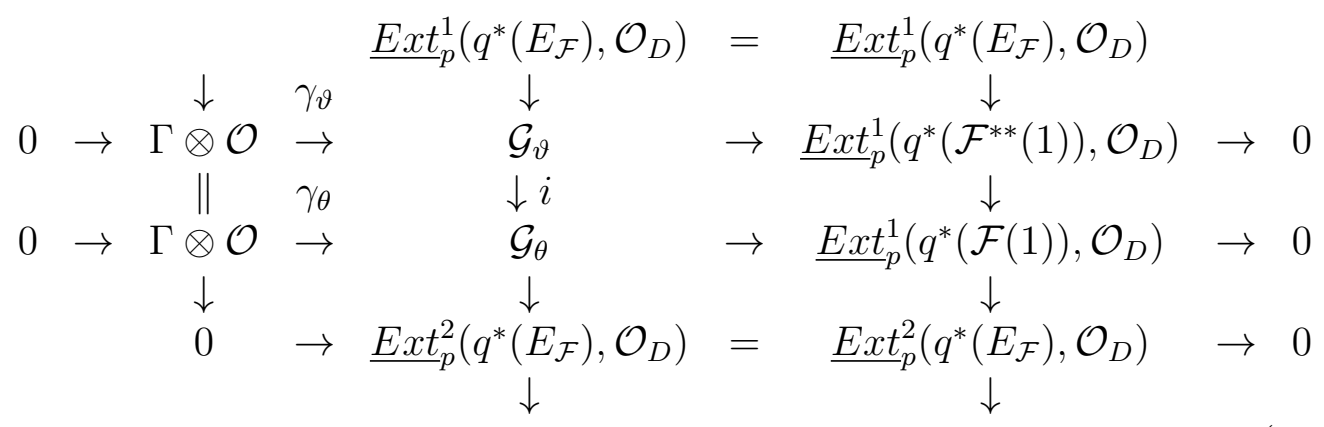

An application of $\underline{\operatorname{Hom}}\left(q^{*}\left(E_{\mathcal{F}}\right),-\right)$ to the resolution of $\mathcal{O}_{D}$ gives (since $q^{*}\left(E_{\mathcal{F})}\right.$ is of codimension 2 and torsion free on its support)

$$
\begin{aligned}
& 0 \rightarrow \underline{\operatorname{Ext}^{1}}\left(q^{*}\left(E_{\mathcal{F}}\right), \mathcal{O}_{D}\right) \rightarrow \underline{\operatorname{Ext}^{2}}\left(q^{*}\left(E_{\mathcal{F}}\right), \mathcal{O}\right) \otimes \mathcal{O}(-1,-1) \rightarrow \\
& \rightarrow \underline{E_{x t}} t^{2}\left(q^{*}\left(E_{\mathcal{F}}\right), \mathcal{O}\right) \rightarrow \underline{E x t^{2}}\left(q^{*}\left(E_{\mathcal{F}}\right), \mathcal{O}_{D}\right) \rightarrow 0
\end{aligned}
$$

where the middle arrow is $\left(s_{D}\right)_{*}$ and $\underline{\operatorname{Hom}}\left(q^{*}\left(E_{\mathcal{F}}\right), \mathcal{O}_{D}\right)=0$. In the same way as one shows 8.3.5 one can show that there is a natural isomorphsim

$$
\underline{\operatorname{Ext}^{2}}\left(q^{*}\left(E_{\mathcal{F}}\right), \mathcal{O}\right) \simeq q^{*}\left(\underline{\operatorname{Ext}^{2}}\left(E_{\mathcal{F}}, \mathcal{O}\right)\right)=q^{*}\left(\check{E_{\mathcal{F}}}\right)
$$

Thus 8.3.9 becomes

$$
\begin{aligned}
& 0 \rightarrow \underline{\operatorname{Ext}}^{1}\left(q^{*}\left(E_{\mathcal{F}}\right), \mathcal{O}_{D}\right) \rightarrow q^{*}\left(\check{E_{\mathcal{F}}}\right) \otimes \mathcal{O}(-1,-1) \rightarrow \\
& \rightarrow \quad q^{*}\left(\check{E}_{\mathcal{F}}\right) \quad \rightarrow \quad \underline{\operatorname{Ext}^{2}}\left(q^{*}\left(E_{\mathcal{F}}\right), \mathcal{O}_{D}\right) \rightarrow 0
\end{aligned}
$$

from which we read

$$
\underline{\operatorname{Ext}^{1}}\left(q^{*}\left(E_{\mathcal{F}}\right), \mathcal{O}_{D}\right)=\underline{\operatorname{Tor}^{1}}\left(q^{*}\left(\check{E_{\mathcal{F}}}\right), \mathcal{O}_{D}\right)=0
$$

and $\underline{\operatorname{Ext}^{2}}\left(q^{*}\left(E_{\mathcal{F}}\right), \mathcal{O}_{D}\right) \simeq q^{*}\left(\check{E_{\mathcal{F}}}\right) \otimes \mathcal{O}_{D}$, therefore by the Grothendieck spectral sequence $\underline{\operatorname{Ext}}_{p}^{2}\left(q^{*}\left(E_{\mathcal{F}}, \mathcal{O}_{D}\right)\right) \simeq q^{*}\left(\check{E}_{\mathcal{F}}\right) \otimes \mathcal{O}_{D}$ and diagram 8.3.8 becomes 


$$
\begin{aligned}
& \begin{array}{cccc}
\downarrow & 0 & 0 \\
& \gamma_{\vartheta} & \downarrow & \\
\downarrow & \downarrow & \\
\mathcal{G}_{\vartheta} & \rightarrow & \underline{E x t}_{p}^{1}\left(q^{*}\left(\mathcal{F}^{* *}(1)\right), \mathcal{O}_{D}\right) \rightarrow & \rightarrow 0
\end{array}
\end{aligned}
$$

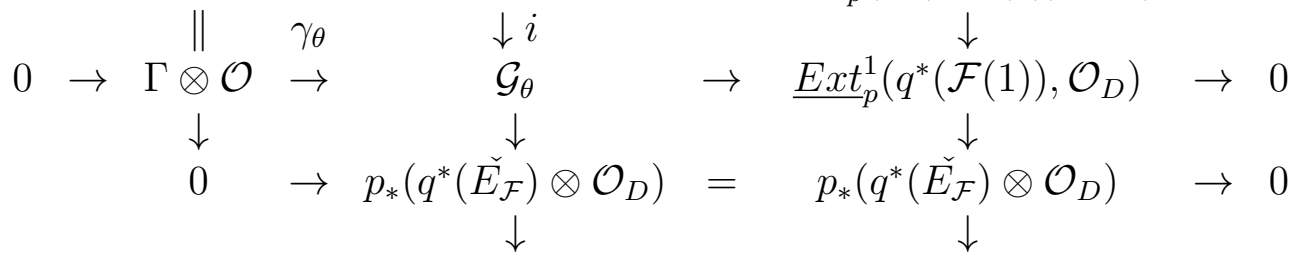

Since $\mathcal{F}^{* *}$ is locally free we have $\gamma_{\vartheta}=e v_{\vartheta}$, thus $\gamma_{\theta}=i \circ e v_{\vartheta}$. Now we apply $p_{*}\left(q^{*}(-) \otimes \mathcal{O}_{D}\right)$ to

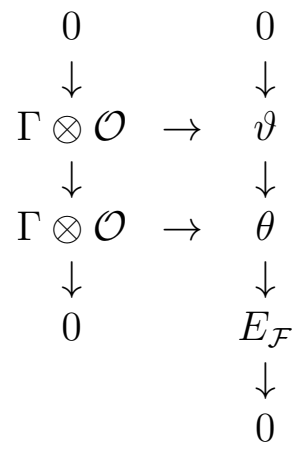

Then we get the left part of 8.3.11 with $\gamma_{\theta}$ replaced by $e v_{\theta}$. Therefore $e v_{\theta}=$ $i \circ e v_{\vartheta}=\gamma_{\theta}$.

This completes the proof.

\subsection{Detecting the spectral scheme}

For a sheaf $\theta$ torsion free on its support with $\operatorname{ch}(\theta)=2 h+3 h^{2}$ one has $\operatorname{ch}\left(\left.\theta\right|_{l}\right)=$ $2 h^{2}$ for all lines $l$ such that $\underline{\operatorname{Tor}}^{1}\left(\theta, \mathcal{O}_{l}\right)=0$, since in that case the resolution of $\mathcal{O}_{l}$ tensored with $\theta$ is still exact and $\operatorname{ch}(\theta(-1))=\operatorname{ch}(\theta) \operatorname{ch}(\mathcal{O}(-1))$. The condition $\underline{\operatorname{Tor}}^{1}\left(\theta, \mathcal{O}_{l}\right)=0$ is satisfied for all lines, which intersect $\operatorname{set} \operatorname{supp}(\theta)$ transversal, thus for a generic line $l, l \cap \operatorname{set} \operatorname{supp}(\theta)$ consists of one or two points, and since $\theta$ is torsion free on its support, $\operatorname{set} \operatorname{supp}(\theta)$ must be a conic or a line, since it is a closed subvariety.

We want to get to know, whether there is a base point free $(\Gamma, \theta) \in \operatorname{Syst}(2 h+$ $\left.3 h^{2}, 2\right)$ such that its spectral scheme has a non reduced component. Our main tool will be the following lemma of LePotier [LP2]. 
Lemma 8.4.1 Let $(\Gamma, \theta) \in \operatorname{Syst}\left(2 h+3 h^{2}, 2\right)$ and $l$ a line in $\mathbb{P}_{2}$ with $l \nsubseteq$ $\operatorname{setsupp}(\theta))$. Then

1. $\check{l} \in \tilde{\Sigma}_{(\Gamma, \theta)}$ if and only if there exists a section $s \in \Gamma$ with

$$
s \in l H^{0}(\theta(-1)) .
$$

2. If $(\Gamma, \theta)$ is furthermore base point free, $\Sigma_{(\Gamma, \theta)}$ is singular on its support at $\check{l}$ if and only if there exists a section $s \in \Gamma$ with

$$
s \in l^{2} H^{0}(\theta(-2)) .
$$

\section{Proof:}

Due to theorem 8.3.1 $\Sigma_{(\Gamma, \theta)}=$ Fitt $_{0}\left(\operatorname{Ext}_{p}^{1}\left(q^{*}(\mathcal{F}(1)), \mathcal{O}_{D}\right)\right)$, which in a local neighborhood $U$ of $\check{l}$ can be computed by using 8.3.1. Restricted to $U, \Gamma \otimes \mathcal{O}$ and $\mathcal{G}_{\theta}$ are locally free of rank 2 . The fibre of $\mathcal{G}_{\theta}$ over $\check{l}$ is isomorphic to $H^{0}\left(\left.\theta\right|_{l}\right)$, and using this isomorphism $\overline{e v}$ maps a section $s \in \Gamma$ at the point $\check{l}$ to $\left.s\right|_{l}$. Thus $\check{l} \in \Sigma_{(\Gamma, \theta)}$ if and only if there is a section in $\Gamma$, that vanishes when restricted to $l$ and this is equivalent to 1 .

If $(\Gamma, \theta)$ is base point free we have

$$
0 \rightarrow \mathcal{F}(-1) \rightarrow \Gamma \otimes \mathcal{O} \rightarrow \theta \rightarrow 0
$$

Since $l \nsubseteq \operatorname{setsupp}(\theta), \operatorname{setsupp}\left(\left.\theta\right|_{l}\right)$ consists of one or two points. Let $p$ be such a point, then if all sections of $\Gamma$ vanish when restricted to $l$ they also vanish when restricted to $p$. But that is a contradiction to 8.4.1 tensored with $\mathcal{O}_{p}$ since $\theta_{p} \neq 0$. Therefore if $(\Gamma, \theta)$ is base point free there can only be a one dimensional subspace of $\Gamma$, whose restriction to $l$ vanishes and this means that $\breve{l}$ does not belong to $F_{i t t}\left(\underline{E x t}_{p}^{1}\left(q^{*}(\mathcal{F}(1)), \mathcal{O}_{D}\right)\right)$, thus we can use lemma 6.3.1. We will use another resolution of $\operatorname{Ext}_{p}^{1}\left(q^{*}(\mathcal{F}(1)), \mathcal{O}_{D}\right)$ to show 2.: We can extend the resolution of $\mathcal{G}$ by $e v$ to a commutative diagram, since $\operatorname{Ext}^{1}\left(\Gamma \otimes \mathcal{O}, H^{0}(\theta(-1)) \otimes \mathcal{O}(-1)\right)=0$

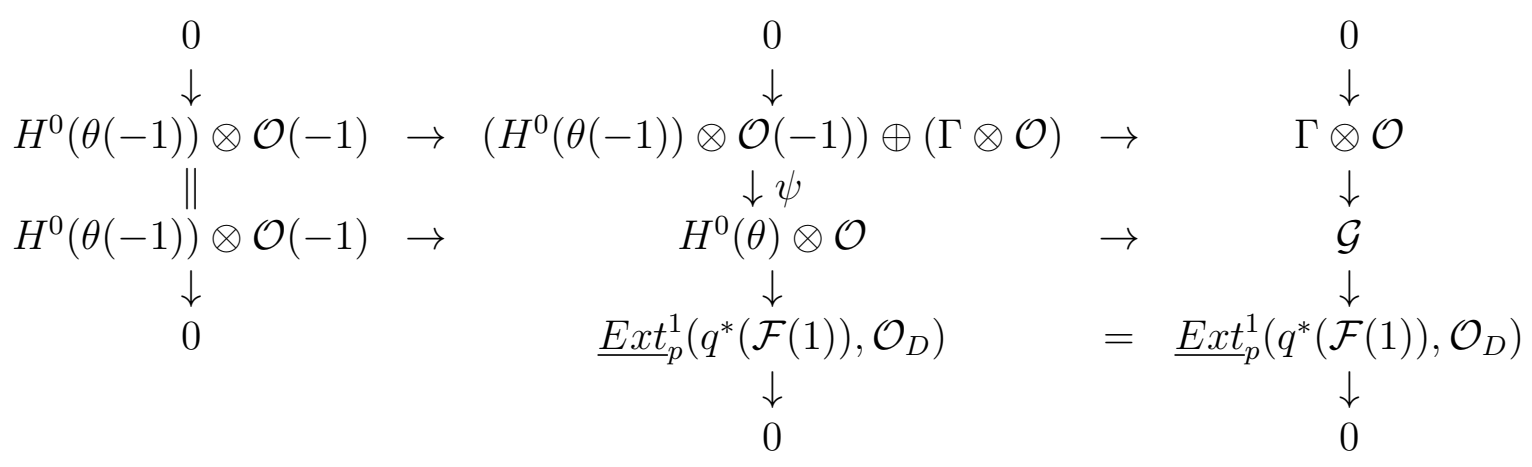


with columns of zeros at the left and at the right. Now we apply lemma 6.3.1 to the middle column to show 2. Let $\check{l} \in \operatorname{Fitt}_{0}\left(\underline{\operatorname{Ext}}_{p}^{1}\left(q^{*}(\mathcal{F}(1)), \mathcal{O}_{D}\right)\right)$. Due to 1 . that means, that there is a $s_{1} \in \Gamma$ and a $\tilde{s}_{1} \in H^{0}(\theta(-1))$ such that $s_{1}=l \tilde{s}_{1}$. Choose homogeneous coordinates $\left(\tilde{\mu}_{1}: \tilde{\mu}_{2}: \tilde{\mu}_{3}\right)$ of $\mathbb{P}_{2}^{*}$ such that $\check{l}=(0: 0: 1)$ and let $U:=\left\{\left(\tilde{\mu}_{1}: \tilde{\mu}_{2}: \tilde{\mu}_{3}\right): \tilde{\mu}_{3} \neq 0\right\}$ with affine coordinates $\left(\mu_{1}, \mu_{2}\right)$. Restricted to $U \psi$ is a map between the two trivial bundles $U \times\left(H^{0}(\theta(-1)) \oplus \Gamma\right)$ and $U \times H^{0}(\theta)$ which at a point $\left(\mu_{1}, \mu_{2}\right)$ that corresponds to a line $\tilde{l}$ is given by $(\tilde{s} \oplus s) \mapsto \tilde{l} \tilde{s}+s$. So $\operatorname{ker}\left(\psi_{(0,0)}\right)=\operatorname{span}\left\{\tilde{s}_{1} \oplus\left(-l \tilde{s}_{1}\right)\right\}$. We compute the differentials of this kernel: One has

$$
\psi_{(\epsilon, 0)}\left(\tilde{s}_{1} \oplus\left(-l \tilde{s}_{1}\right)\right)=(m-l) \tilde{s}_{1}
$$

where $m=\epsilon x+z$. Since $m-l=\epsilon x \in H^{0}(\mathcal{O}(1))$ we get

$$
\frac{\partial \psi}{\partial \mu_{1}(0,0)}\left(\tilde{s}_{1} \oplus\left(-l \tilde{s}_{1}\right)\right)=x \tilde{s}_{1}
$$

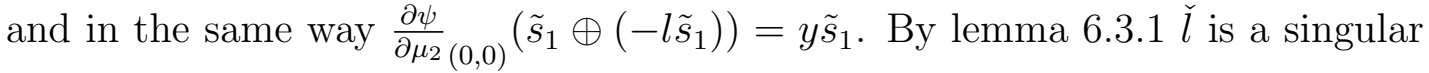
point if and only if

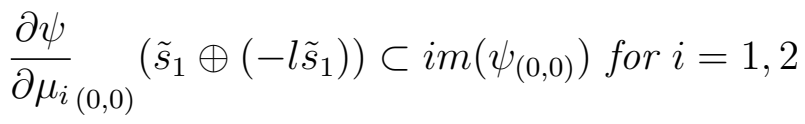

with $s_{2} \in \Gamma$ such that $\operatorname{span}\left\{s_{1}, s_{2}\right\}=\Gamma$ one has

$$
i m\left(\psi_{(0,0)}\right)=\operatorname{span}\left\{s_{2}\right\} \oplus l H^{0}(\theta(-1))
$$

Now if $s_{1}=l^{2} \hat{s}_{1}$ then $\tilde{s}_{1}=l \hat{s}_{1}$ and therefore $x \tilde{s}_{1}=l\left(x \hat{s}_{1}\right) \in l H^{0}(\theta(-1))$ and $y \tilde{s}_{1} \in l H^{0}(\theta(-1))$, thus $\Sigma_{(\Gamma, \theta)}$ is singular on its support at $\check{l}$. If on the other hand $\Sigma_{(\Gamma, \theta)}$ is singular on its support at $\breve{l}, 8.4 .3$ is satisfied and therefore

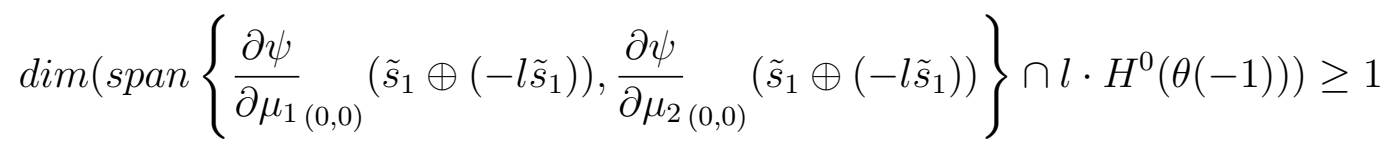

thus there are $c_{1}, c_{2} \in \mathbb{C}$ such that

$$
{\frac{\partial \psi}{\partial\left(c_{1} \mu_{1}+c_{2} \mu_{2}\right)}}_{(0,0)}\left(\tilde{s}_{1} \oplus\left(-l \tilde{s}_{1}\right)\right)=\left(c_{1} x+c_{2} y\right) \tilde{s}_{1} \in l H^{0}(\theta(-1)
$$


But that means that $\tilde{s}_{1}=l \hat{s}_{1}$, which is $s_{1}=l^{2} \hat{s}_{1}$.

This completes the proof.

Now we will investigate the spectral schemes of all $(\Gamma, \theta) \in \operatorname{Syst}\left(2 h+3 h^{2}, 2\right)$. Since $\theta$ is a sheaf of codimension 1 , one has $\operatorname{Tor}^{1}\left(\mathcal{O}_{l}, \theta\right)=0$ for a general line $l$. Because of $c_{1}(\theta)=2$ one gets from tensoring the resolution of $\mathcal{O}_{l}$ with $\theta$ that $\operatorname{ch}\left(\left.\theta\right|_{l}\right)=2 h^{2}$. Therefore $l$ intersects $\operatorname{set} \operatorname{supp}(\theta)$ in at most 2 points. Therefore we have to consider the three cases, where $\operatorname{set} \operatorname{supp}(\theta)$ is either a nonsingular conic or a singular conic or a line.

\subsubsection{Nonsingular conics}

Let $C \subset \mathbb{P}_{2}$ be a nonsingular conic. $C$ is isomorphic to $\mathbb{P}_{1}$. Let $i: C \rightarrow \mathbb{P}_{1}$ be such an isomorphism. If for a sheaf $\theta$ of codimension one, torsion free on its support $\operatorname{ch}(\theta)=2 h+r h^{2}$ and $\operatorname{setsupp}(\theta)=C, \operatorname{supp}(\theta)$ is reduced, therefore $\theta$ is also an $\mathcal{O}_{C}$-modul of rank 1 , and since $\theta$ is torsion free on its support, it is even locally free over $\mathcal{O}_{C}$. Therefore there is a $j \in \mathbb{Z}$ such that $\theta=i^{*}\left(\mathcal{O}_{\mathbb{P}_{1}}(j)\right)$. We compute $j$, such that $\operatorname{ch}\left(i^{*}\left(\mathcal{O}_{\mathbb{P}_{1}}(j)\right)\right)=2 h+3 h^{2}$. For even $j, i^{*}\left(\mathcal{O}_{\mathbb{P}_{1}}(j)\right)=i^{*}\left(\mathcal{O}_{\mathbb{P}_{1}}(2 k)\right)$ is a restriction of $\mathcal{O}_{\mathbb{P}_{2}}(k)$ :

$$
0 \rightarrow \mathcal{O}(k-2) \stackrel{f}{\rightarrow} \mathcal{O}(k) \rightarrow \mathcal{O}_{C}(k) \rightarrow 0
$$

where $f \in H^{0}(\mathcal{O}(2))$ such that $C=\left\{p \in \mathbb{P}_{2} \mid f(p)=0\right\}$. So $\mathcal{O}_{C}(k)=i^{*}\left(\mathcal{O}_{\mathbb{P}_{1}}(2 k)\right)$. Any inclusion $\mathcal{O}_{\mathbb{P}_{1}}(2 k-1) \hookrightarrow \mathcal{O}_{\mathbb{P}_{1}}(2 k)$ induces an inclusion $i^{*}\left(\mathcal{O}_{\mathbb{P}_{1}}(2 k-1)\right) \hookrightarrow$ $\mathcal{O}_{C}(k)$. With this map we can extend 8.4.4 to a diagram

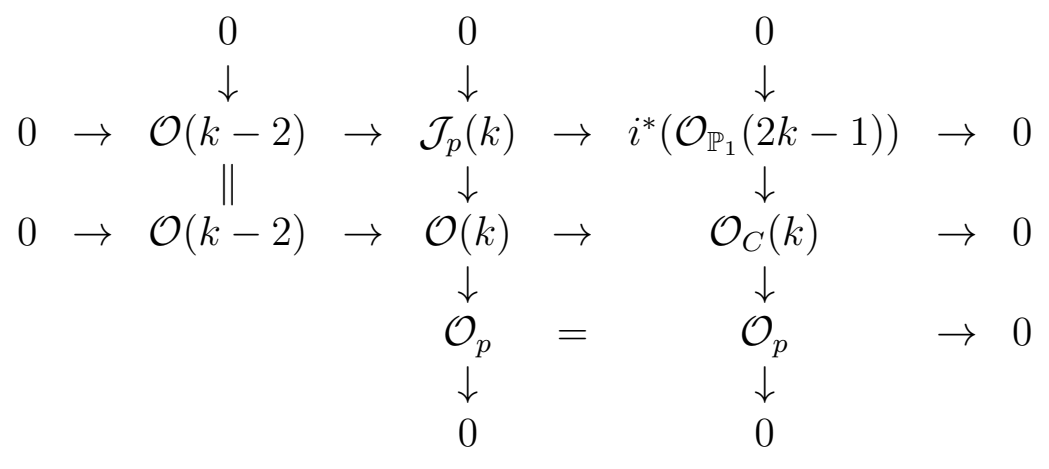

where $p$ is a point depending on the choices of the inclusion $\mathcal{O}_{\mathbb{P}_{1}}(2 k-1) \hookrightarrow$ $\mathcal{O}_{\mathbb{P}_{1}}(2 k)$ and the isomorphism $i$. We compute

$$
\operatorname{ch}\left(i^{*}\left(\mathcal{O}_{C}(k)\right)=\operatorname{ch}(\mathcal{O}(k))-\operatorname{ch}(\mathcal{O}(k-2))=2 h+(2 k-2) h^{2},\right.
$$




$$
\operatorname{ch}\left(i^{*}\left(\mathcal{O}_{\mathbb{P}_{1}}(2 k-1)\right)\right)=\operatorname{ch}\left(\mathcal{O}_{C}(k)\right)-\operatorname{ch}\left(\mathcal{O}_{p}\right)=2 h+(2 k-3) h^{2} .
$$

Thus the only $\theta$ supported on a nonsingular conic that can occur in a $(\Gamma, \theta) \in$ $\operatorname{Syst}\left(2 h+3 h^{2}, 2\right)$ is $\theta=i^{*}\left(\mathcal{O}_{\mathbb{P}_{1}}(5)\right)$.

Since $C$ is isomorphic to $\mathbb{P}_{1}$, we can reformulate the conditions of lemma 8.4.1 using $i_{*} \theta$ on $\mathbb{P}_{1}$ :

Lemma 8.4.2 Let $\theta=i^{*}\left(\mathcal{O}_{\mathbb{P}_{1}}(j)\right)$ with $j>0$.

1. Let $l$ be transversal to $C$ such that $l \cap C=p \cup q$. Then $s \in l^{k} H^{0}(\theta(-k))$ if and only if $i_{*}(s)$ has a zero of multiplicity at least $k$ at $i(p)$ and $i(q)$.

2. Let $l$ be tangent to $C$, such that $l \cap C=p$. Then $s \in l^{k} H^{0}(\theta(-k))$ if and only if $i_{*}(s)$ has a zero of multiplicity at least $2 k$ at $i(p)$.

\section{Proof:}

$l$ is a section of $\mathcal{O}_{\mathbb{P}_{2}}(1)$ and therefore a representative of a section $\tilde{l}$ of $\mathcal{O}_{C}(1)$. $i_{*}(\tilde{l})$ is a section of $\mathcal{O}_{\mathbb{P}_{1}}(2)$. If $l$ is transversal to $C i_{*}(\tilde{l})$ has single zeros as $i(p)$ and $i(q)$, if $l$ is tangent to $C$ it has a double zero at $i(p)$. Thus if $s=l^{k} \tilde{s}$ with $\tilde{s} \in H^{0}(\theta(-k)) i_{*}(s)$ has zeros of order $k$ at $i(p)$ and $i(q)$ or a zero of order $2 k$ at $i(p)$ respectively.

This completes the proof.

Proposition 8.4.3 Let $(\Gamma, \theta) \in \operatorname{Syst}\left(2 h+3 h^{2}, 2\right)$ be base point free. If $\operatorname{setsupp}(\theta)=C$ is a nonsingular conic, then $\Sigma_{(\Gamma, \theta)}$ is reduced.

\section{Proof:}

Since $(\Gamma, \theta)$ is base point free, the evaluation map $\overline{e v}$ is surjective:

$$
0 \rightarrow \mathcal{F}^{*}(-1) \rightarrow \Gamma \otimes \mathcal{O} \stackrel{\overline{e v}}{\rightarrow} \theta \rightarrow 0
$$

and therefore its restriction to any point $q \in C$ as well: $\overline{e v}_{q}: \Gamma \otimes \mathcal{O}_{q} \rightarrow \theta_{q} \rightarrow 0$. Since $C$ is a nonsingular conic one has $\theta_{q}=\mathcal{O}_{q}$, thus up to a factor there is a unique section $s_{q} \in \Gamma$ such that $\overline{e v}_{q}\left(s_{q} \otimes \mathcal{O}_{q}\right)=0$. The pullback $i^{*}(\Gamma)$ is a 2 dimensional subspace of $H^{0}\left(\mathcal{O}_{\mathbb{P}_{1}}(5)\right)$, the zero divisors of the projectivisation of $i^{*}(\Gamma)$ form a pencil of divisors. The theorem of Bertini (see [GH],p.137) states, that a general element of such a pencil consists of 5 different points. Thus for a 
general point $q \in C, i^{*}\left(s_{q}\right)$ has 5 zeros of multiplicity 1 . Since this $s_{q}$ is unique up to a factor, there is no line $l$ through $q$ such that $\Sigma_{(\Gamma, \theta)}$ is singular on its support at $\check{l}$ due to lemma 8.4.2. But that means, that $\Sigma_{(\Gamma, \theta)}$ restricted to $\check{q}$ consists of nonsingular points only and that contradicts, that $\Sigma_{(\Gamma, \theta)}$ is non reduced.

That completes the proof.

\subsubsection{Singular conics}

Now we investigate coherent systems $(\Gamma, \theta) \in \operatorname{Syst}\left(2 h+3 h^{2}, 2\right)$ such that $\operatorname{setsupp}(\theta)$ is a singular conic $C=t_{1} \cup t_{2}, t_{1} \neq t_{2}$ with $t_{1}$ being the zero set of $g_{1} \in H^{0}(\mathcal{O}(1))$ and $t_{2}$ the zero set of $g_{2} \in H^{0}(\mathcal{O}(1))$ respectively. Let $p=t_{1} \cap t_{2}$. We will see that any such $\theta$ is either $\mathcal{O}_{t_{1}}(i) \oplus \mathcal{O}_{t_{2}}(4-i)$ or a sheaf locally free over $\mathcal{O}_{C}$, the structure sheaf of the scheme given by the ideal $\left(g_{1} g_{2}\right)$. Finally we show that there is no base point free $(\Gamma, \theta)$ with $\operatorname{set} \operatorname{supp}(\theta)=C$ such that $\Sigma_{(\Gamma, \theta)}$ is non reduced.

Lemma 8.4.4 Let $\theta$ be coherent sheaf on $\mathbb{P}_{2}$ torsion free on its support with $\operatorname{ch}(\theta)=2 h+3 h^{2}$ and setsupp $(\theta)=t_{1} \cup t_{2}=C$ a singular conic, then there exists $i_{j} \in \mathbb{Z}$ for $j=1,2$ such that

$$
0 \rightarrow \mathcal{O}_{t_{3-j}}\left(i_{j}\right) \rightarrow \theta \rightarrow \mathcal{O}_{t_{j}}\left(4-i_{j}\right) \rightarrow 0
$$

with $\max \left\{i_{1}, i_{2}\right\} \geq 2$.

\section{Proof:}

Consider the restriction of $\theta$ to $t_{j}$. This map is surjective, since it results from tensoring the resolution of $\mathcal{O}_{t_{j}}$ with $\theta$. Denote its kernel, which is supported on $t_{3-j}$, by $\mathcal{K}_{j}$ :

$$
\left.0 \rightarrow \mathcal{K}_{j} \rightarrow \theta \rightarrow \theta\right|_{t_{j}} \rightarrow 0
$$

If we apply $\underline{\operatorname{Hom}}(-, \mathcal{O})$ to this sequence, we find

$$
\underline{\operatorname{Ext}}^{2}(\theta, \mathcal{O}) \rightarrow \underline{\operatorname{Ext}^{2}}\left(\mathcal{K}_{j}, \mathcal{O}\right) \rightarrow 0
$$

By lemma 2.4.2 $\mathcal{K}_{j}$ is torsion free on its support since $\theta$ is, so $\mathcal{K}_{j}=\mathcal{O}_{t_{3-j}}\left(m_{j}\right)$. Furthermore the only point, where $\left.\theta\right|_{t_{j}}$ can have torsion is $p$, because any other subsheaf of $\left.\theta\right|_{t_{j}}$ supported on a Hilbertscheme of points on $t_{j} \backslash p$ would also be a 
torsion subsheaf of $\theta$. Let $T^{j}$ be the torsion subsheaf of $\left.\theta\right|_{t_{j}}$. The map $T^{j} \hookrightarrow \mathcal{O}_{t_{j}}$ extends 8.4.5 uniquely to the diagram

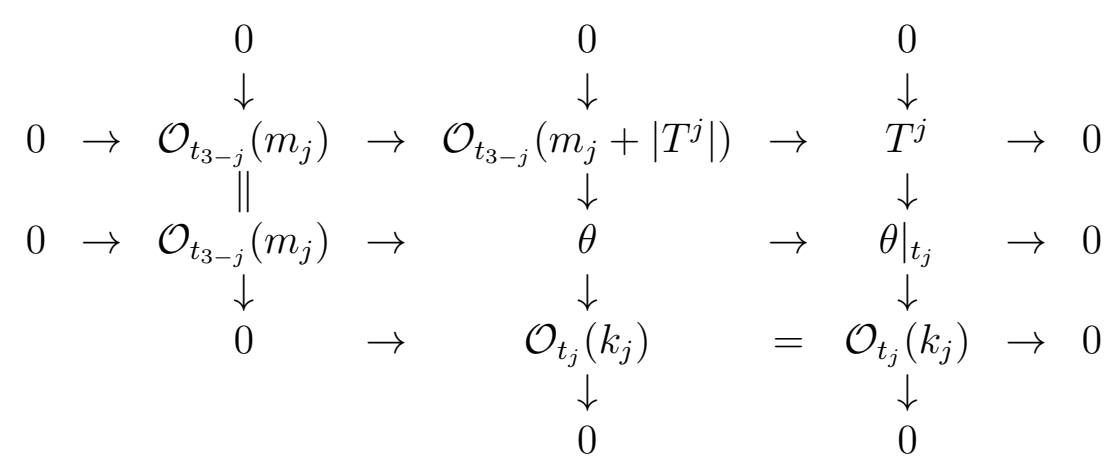

where $\left|T^{j}\right|$ is given by $\operatorname{ch}\left(T^{j}\right)=\left|T^{j}\right| h^{2}$. Taking $k_{j}=4-i_{j}$, the middle column is the sequence we are looking for, since $\operatorname{ch}\left(\mathcal{O}_{t_{3-j}}\left(m_{j}+\left|T^{j}\right|\right)\right)+\operatorname{ch}\left(\mathcal{O}_{t_{j}}\left(k_{j}\right)\right)=$ $2 h+3 h^{2}$.

Now suppose $\max \left\{i_{1}, i_{2}\right\}<2$. Then $\operatorname{ch}\left(\mathcal{O}_{t_{1}}\left(m_{2}\right)\right) \leq h+\frac{1}{2} h^{2}$ and the middle row of the diagram for $j=2$ gives and $\operatorname{ch}\left(\left.\theta\right|_{t_{2}}\right) \geq h+\frac{5}{2} h^{2}$. But tensoring

$$
0 \rightarrow \mathcal{O}_{t_{2}}\left(i_{1}\right) \rightarrow \theta \rightarrow \mathcal{O}_{t_{1}}\left(4-i_{1}\right) \rightarrow 0
$$

with $\mathcal{O}_{t_{2}}$ gives

$$
\left.0 \rightarrow \mathcal{O}_{t_{2}}\left(i_{1}\right) \rightarrow \theta\right|_{t_{2}} \rightarrow \mathcal{O}_{p} \rightarrow 0
$$

and therefore $\max \left\{i_{1}, i_{2}\right\}<2$ would imply $\operatorname{ch}\left(\mathcal{O}_{p}\right) \geq 2 h^{2}$.

This completes the proof.

Lemma 8.4.5 $\operatorname{dim}\left(\operatorname{Ext}^{1}\left(\mathcal{O}_{t_{j}}\left(4-i_{j}\right), \mathcal{O}_{t_{3-j}}\left(i_{j}\right)\right)\right)=1$ and any $\theta \in \operatorname{Ext}^{1}\left(\mathcal{O}_{t_{j}}(4-\right.$ $\left.\left.i_{j}\right), \mathcal{O}_{t_{3-j}}\left(i_{j}\right)\right)$ is torsion free on its support for $j=1,2, i_{j} \in \mathbb{Z}$.

\section{Proof:}

If we dualize the resolution of $\mathcal{O}_{t_{j}}\left(4-i_{j}\right)$, we find that $\underline{\operatorname{Ext}} t^{1}\left(\mathcal{O}_{t_{j}}\left(4-i_{j}\right), \mathcal{O}\right)=$ $\mathcal{O}_{t_{j}}\left(i_{j}-3\right)$. Then we apply $\underline{\operatorname{Hom}}\left(\mathcal{O}_{t_{j}}\left(4-i_{j}\right),-\right)$ to the resolution of $\mathcal{O}_{t_{3-j}}\left(i_{j}\right)$ and find since $\underline{\operatorname{Hom}}\left(\mathcal{O}_{t_{j}}\left(4-i_{j}\right), \mathcal{O}_{t_{3-j}}\left(i_{j}\right)\right)=0$

$$
\underline{\operatorname{Ext}^{1}}\left(\mathcal{O}_{t_{j}}\left(4-i_{j}\right), \mathcal{O}_{t_{3-j}}\left(i_{j}\right)\right)=\mathcal{O}_{p}
$$

Using the Grothendieck spectral sequence of $H^{0} \circ \underline{\operatorname{Hom}}\left(\mathcal{O}_{t_{j}}\left(4-i_{j}\right),-\right)$ gives 
$\operatorname{dim}\left(\operatorname{Ext}^{1}\left(\mathcal{O}_{t_{j}}\left(4-i_{j}\right), \mathcal{O}_{t_{3-j}}\left(i_{j}\right)\right)\right)=h^{0}\left(\underline{\operatorname{Ext}^{1}}\left(\mathcal{O}_{t_{j}}\left(4-i_{j}\right), \mathcal{O}_{t_{3-j}}\left(i_{j}\right)\right)\right)=h^{0}\left(\mathcal{O}_{p}\right)=1$

Any $\theta \in \operatorname{Ext}^{1}\left(\mathcal{O}_{t_{j}}\left(4-i_{j}\right), \mathcal{O}_{t_{3-j}}\left(i_{j}\right)\right)$ is torsion free on its support: Dualizing

$$
0 \rightarrow \mathcal{O}_{t_{3-j}}\left(i_{j}\right) \rightarrow \theta \rightarrow \mathcal{O}_{t_{j}}\left(4-i_{j}\right) \rightarrow 0
$$

leads to

$$
\underline{\operatorname{Ext}}{ }^{2}\left(\mathcal{O}_{t_{j}}\left(4-i_{j}\right), \mathcal{O}\right) \rightarrow \underline{\operatorname{Ext}}{ }^{2}(\theta, \mathcal{O}) \rightarrow \underline{\operatorname{Ext}^{2}}\left(\mathcal{O}_{t_{3-j}}\left(i_{j}\right), \mathcal{O}\right) \rightarrow 0
$$

But $\mathcal{O}_{t_{3-j}}\left(i_{j}\right)$ and $\mathcal{O}_{t_{j}}\left(4-i_{j}\right)$ are torsion free on their support, thus $\underline{\operatorname{Ext}}^{2}\left(\mathcal{O}_{t_{j}}(4-\right.$ $\left.\left.i_{j}\right), \mathcal{O}\right)=\underline{\operatorname{Ext}^{2}}\left(\mathcal{O}_{t_{3-j}}\left(i_{j}\right), \mathcal{O}\right)=0$ by lemma 2.4 .2 and therefore $\theta$ is torsion free on its support as well.

This completes the proof.

As in the nonsingular case some of the sheaves locally free over $\mathcal{O}_{C}$ are quotients of sheaves locally free over $\mathcal{O}_{\mathbb{P}_{2}}$ :

$$
0 \rightarrow \mathcal{O}(k-2) \stackrel{g_{1} g_{2}}{\rightarrow} \mathcal{O}(k) \rightarrow \mathcal{O}_{C}(k) \rightarrow 0
$$

with $\operatorname{ch}\left(\mathcal{O}_{C}(k)\right)=2 h+(2 k-2) h^{2}$. We can extend 8.4.6 to

$$
\begin{array}{ccccccc}
0 & & 0 & & 0 \\
\downarrow & g_{j} & \downarrow & & \downarrow & & \\
0 \rightarrow \mathcal{O}(k-2) & \rightarrow & \mathcal{O}(k-1) & \rightarrow & \left.\mathcal{O}_{t_{j}}(k-1)\right) & \rightarrow & 0 \\
\| & g_{1} g_{2} & \downarrow g_{3-j} & & \downarrow & & \\
0 \rightarrow \mathcal{O}(k-2) & \rightarrow & \mathcal{O}(k) & \rightarrow & \mathcal{O}_{C}(k) & \rightarrow & 0 \\
& & \downarrow & & \downarrow & & \\
& & \mathcal{O}_{t_{3-j}}(k) & & \mathcal{O}_{t_{3-j}}(k) & \rightarrow & 0 \\
& & & & \downarrow & & \\
& & & & 0 & &
\end{array}
$$

Thus $\mathcal{O}_{C}(k) \in \operatorname{Ext}^{1}\left(\mathcal{O}_{t_{3-j}}(k), \mathcal{O}_{t_{j}}(k-1)\right)$ is a nontrivial extension for $j=1,2$. 
Lemma 8.4.6 Let $\theta$ be a coherent sheaf on $\mathbb{P}_{2}$ torsion free on its support with $\operatorname{ch}(\theta)=2 h+3 h^{2}$ and setsupp $(\theta)=t_{1} \cup t_{2}=C$ a singular conic, then $\theta$ is either $\mathcal{O}_{t_{1}}(k) \oplus \mathcal{O}_{t_{2}}(4-k)$ with $k \in \mathbb{Z}$ or a sheaf locally free over $\mathcal{O}_{C}$, for which there is a $i \in \mathbb{Z}$ and a Hilbert scheme of points $\eta$ with $\operatorname{ch}(\eta)=(2 i-3) h^{2}$ either on $t_{1}$ or on $t_{2}$ and $\eta \cap p=\emptyset$ such that

$$
0 \rightarrow \theta \rightarrow \mathcal{O}_{C}(i+1) \rightarrow \mathcal{O}_{\eta} \rightarrow 0
$$

\section{Proof:}

Due to lemma 8.4.4 we have

$$
0 \rightarrow \mathcal{O}_{t_{1}}\left(i_{2}\right) \rightarrow \theta \rightarrow \mathcal{O}_{t_{2}}\left(4-i_{2}\right) \rightarrow 0
$$

Assume $i=i_{2} \geq 2$. Consider

$$
0 \rightarrow \mathcal{O}_{t_{1}}(i) \rightarrow \mathcal{O}_{C}(i+1) \rightarrow \mathcal{O}_{t_{2}}(i+1) \rightarrow 0
$$

A choice of a Hilbert scheme $\eta$ with $c h(\eta)=(2 i-3) h^{2}$ on $t_{2}$ defines a unique inclusion $\mathcal{O}_{t_{2}}(4-i) \hookrightarrow \mathcal{O}_{t_{2}}(i+1)$, which extends the sequence 8.4.8 uniquely to a commutative diagram :

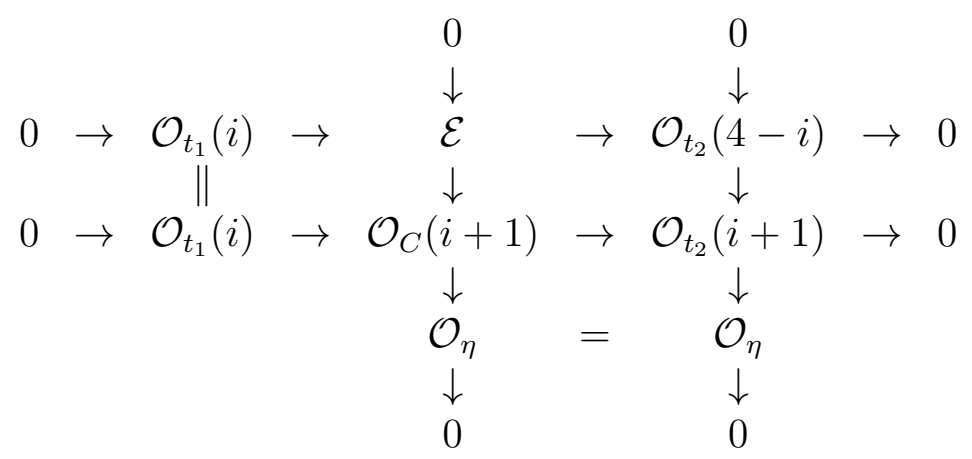

Due to lemma 8.4.5 there is only one nontrivial extension of $\mathcal{O}_{t_{2}}(4-i)$ by $\mathcal{O}_{t_{1}}(i)$. If $\eta \cap p=\emptyset$ we see, that $\mathcal{E}$ is this nontrivial extension, since tensoring the middle column with $\mathcal{O}_{p}$ gives $\mathcal{E}_{p}=\mathcal{O}_{p}$. If $\eta \cap p \neq \emptyset$ we get the trivial extension: We tensor the whole diagram 8.4 .9 with $\mathcal{O}_{p}$ and get

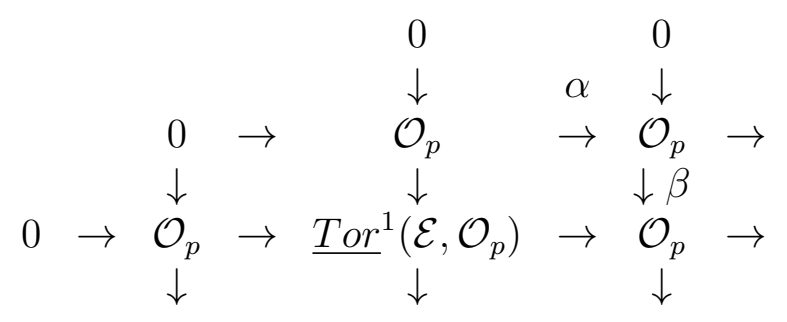


Since $\beta \circ \alpha$ is injective $\operatorname{Tor}^{1}\left(\mathcal{E}, \mathcal{O}_{p}\right)$ must be unequal to $\mathcal{O}_{p}$, therefore we obtain from tensoring the middle column of 8.4 .9 with $\mathcal{O}_{p}$ that $\mathcal{E}_{p} \neq \mathcal{O}_{p}$. That contradicts that $\mathcal{E}$ is the nontrivial extension.

The nontrivial extension is locally free over $\mathcal{O}_{C}$ : since $\eta \cap p=\emptyset$ there is a open neighborhood $U_{p}$ of $p$ with $U_{p} \cap \eta=\emptyset$. Thus $\left.\left.\theta\right|_{U_{p}} \simeq \mathcal{O}_{C}\right|_{U_{p}}$. For any other point $q \in C$ there open neighborhood $U_{q}$ such that $\left.\mathcal{O}_{C}(i+1)\right|_{U_{q}}=\left.\mathcal{O}_{t_{j}}\right|_{U_{q}}$. Thus restricted to $U_{q} 8.4 .7$ becomes

$$
\left.\left.\left.0 \rightarrow \theta\right|_{U_{q}} \rightarrow \mathcal{O}_{t_{j}}\right|_{U_{q}} \rightarrow \mathcal{O}_{\eta}\right|_{U_{q}} \rightarrow 0
$$

Therefore $\left.\left.\theta\right|_{U_{q}} \simeq \mathcal{O}_{C}\right|_{U_{q}}$, so $\theta$ is locally free over $\mathcal{O}_{C}$.

This completes the proof.

Remark: Since in the above proof two Hilbert schemes $\eta, \xi$ with $\eta \cap p=0$ $\xi \cap p=0$ and $\left|\mathcal{O}_{\eta}\right|=\left|\mathcal{O}_{\xi}\right|$ define the same sheaf $\theta$, we will denote such a nontrivial extension by $\mathcal{J}_{\left|\mathcal{O}_{\eta}\right|}^{j}(i+1)$ if $\eta \subset t_{j}$. Note that if for $\theta$ not a direct sum of line bundles with $i_{1}, i_{2} \geq 2$ there are Hilbert schemes $\eta_{j}$ on $t_{j}$ for $j=1,2$ such that $\theta=\mathcal{J}_{\mid \mathcal{O}_{\eta_{1} \mid}}^{1}\left(i_{1}+1\right)=\mathcal{J}_{\left|\mathcal{O}_{\eta_{2}}\right|}^{2}\left(i_{2}+1\right)$

To show that there is no base point free coherent system $(\Gamma, \theta) \in \operatorname{Syst}\left(2 h+3 h^{2}, 2\right)$ supported on a singular conic such that $\Sigma_{(\Gamma, \theta)}$ has a non reduced component we need the following

Lemma 8.4.7 Let $(\Gamma, \theta) \in \operatorname{Syst}\left(2 h+3 h^{2}, 2\right)$ with setsupp $(\theta)=C=t_{1} \cup t_{2}$ and $\theta$ torsion free on its support. If $(\Gamma, \theta)$ is base point free, then for the sequences of coherent systems

$$
0 \rightarrow\left(\Gamma_{j}^{\prime}, \mathcal{O}_{t_{3-j}}\left(i_{j}\right)\right) \rightarrow(\Gamma, \theta) \rightarrow\left(\Gamma_{j}^{\prime \prime}, \mathcal{O}_{t_{j}}\left(4-i_{j}\right)\right) \rightarrow 0
$$

induced by the sequences of lemma 8.4.4 one has $\operatorname{dim}\left(\Gamma_{j}^{\prime \prime}\right)=2$.

\section{Proof:}

$\Gamma_{j}^{\prime}$ and $\Gamma_{j}^{\prime \prime}$ are defined as follows: Denenoting the maps from lemma 8.4 .4 by $f_{J}^{\prime}$ : $\mathcal{O}_{t_{3-j}}\left(i_{j}\right) \rightarrow \theta$ and $f_{j}^{\prime \prime}: \theta \rightarrow \mathcal{O}_{t_{j}}\left(4-i_{j}\right)$ then $\Gamma_{J}^{\prime}=\left(f_{*}^{\prime}\right)^{-1}(\Gamma)$ and $\Gamma_{j}^{\prime \prime}=f_{*}^{\prime \prime}(\Gamma)$. The short exact sequences of coherent systems means, that we have a commutative diagram

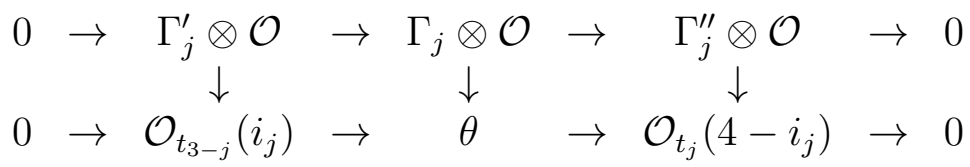


Since $(\Gamma, \theta)$ is base point free, the middle down arrow is surjective and therefore the right one as well. Let $E$ be its kernel:

$$
0 \rightarrow E \rightarrow \Gamma_{j}^{\prime \prime} \otimes \mathcal{O} \rightarrow \mathcal{O}_{t_{j}}\left(4-i_{j}\right) \rightarrow 0
$$

By dualizing 8.4.12 we see that $\underline{\operatorname{Ext}} t^{1}(E, \mathcal{O})=\underline{\operatorname{Ext}^{2}}(E, \mathcal{O})=0$, thus $E$ is locally free of rank 2 , therefore $\operatorname{dim}_{\mathcal{O}_{q}} E_{q}=2$ for any $q \in \mathbb{P}_{2}$. Now we tensor 8.4.12 with $\mathcal{O}_{q}$ with $q \in t_{j}$ :

$$
0 \rightarrow \mathcal{O}_{q} \rightarrow E_{q} \rightarrow \Gamma_{j}^{\prime \prime} \otimes \mathcal{O}_{q} \rightarrow \mathcal{O}_{q} \rightarrow 0
$$

Since $\operatorname{dim}_{\mathcal{O}_{q}} E_{q}=2$ we must have $\operatorname{dim}\left(\Gamma_{j}^{\prime \prime}\right)=2$.

This completes the proof.

$\operatorname{dim}\left(\Gamma^{\prime \prime}\right)=2$ implies, that we can only have those $\theta$, such that the sheaves $\theta / \mathcal{O}_{t_{3-j}}\left(i_{j}\right)$ have at least a 2 dimensional space of global sections, which means $i_{j} \leq 3$ for $j=1,2$. Thus we get:

Corollary 8.4.8 Let $C=t_{1} \cup t_{2}$ be a singular conic. Then there are only 7 sheaves $\theta$, that can occur in a base point free $(\Gamma, \theta) \in \operatorname{Syst}\left(2 h+3 h^{2}, 2\right)$ with $\operatorname{set} \operatorname{supp}(\theta)=C$. These are:

$$
\mathcal{J}_{1}^{j}(3), \quad \mathcal{J}_{3}^{j}(4), \quad \mathcal{O}_{t_{1}}(2) \oplus \mathcal{O}_{t_{2}}(2), \quad \mathcal{O}_{t_{j}}(1) \oplus \mathcal{O}_{t_{3-j}}(3)
$$

with $j=1,2$.

Proposition 8.4.9 For any base point free $(\Gamma, \theta) \in \operatorname{Syst}\left(2 h+3 h^{2}, 2\right)$ with $\operatorname{set} \operatorname{supp}(\theta)=C=t_{1} \cup t_{2}$ a singular conic, $\Sigma_{(\Gamma, \theta)}$ is reduced.

\section{Proof:}

We have to consider the 7 sheaves of corollary 8.4.8. If $\Sigma_{(\Gamma, \theta)}$ has a non reduced component, then there is a line $l \subset \mathbb{P}_{2}$ with $t_{1} \neq l \neq t_{2}$ and a section $s \in \Gamma$ such that $s=l^{2} \tilde{s}$ with $\tilde{s} \in H^{0}(\theta(-2))$ due to lemma 8.3.1. If $\theta=\mathcal{J}_{3}^{j}(4)$ or $\theta=\mathcal{O}_{t_{j}}(1) \oplus \mathcal{O}_{t_{3-j}}(3)$, which means that we have a sequence

$$
0 \rightarrow \mathcal{O}_{t_{3-j}}(3) \rightarrow \theta \rightarrow \mathcal{O}_{t_{j}}(1) \rightarrow 0
$$

then $H^{0}(\theta(-2))=H^{0}\left(\mathcal{O}_{t_{3-j}}(1)\right)$. Thus if there is a section $s=l^{2} \tilde{s}$, its image in $H^{0}\left(\mathcal{O}_{t_{j}}(1)\right)$ is zero, which means we have for the coherent system of lemma 8.4.7 
$\left(\Gamma_{j}^{\prime \prime}, \mathcal{O}_{t_{j}}\left(4-i_{j}\right)\right) \operatorname{dim}\left(\Gamma_{j}^{\prime \prime}\right)<2$ for $j=1$ or $j=2$, which is a contradiction to the non existence of a base point. Therefore the sheaves left to be considered are $\mathcal{J}_{1}^{j}(3)$ and $\mathcal{O}_{t_{1}}(2) \oplus \mathcal{O}_{t_{2}}(2)$

Suppose $\Sigma_{(\Gamma, \theta)}$ is a double conic, whose reduction $D$ is nonsingular. Take 3 points $r_{1}, \ldots, r_{3} \in D$ such that $\check{r}_{1} \cup \check{r}_{2} \cup \check{r}_{3}$ intersects each of the lines $t_{1}, t_{2}$ at 3 different points. Due to lemma 8.3.1 there are three different elements in $\mathbb{P}(\Gamma)$ such that their images in $\mathbb{P}\left(\Gamma^{\prime \prime}\right)$ have a double zero. But either $\Gamma^{\prime \prime}=H^{0}\left(\mathcal{O}_{t_{1}}(1)\right)$ and there is no such element in $\mathbb{P}\left(\Gamma^{\prime \prime}\right)$ or the zeros of $\mathbb{P}\left(\Gamma^{\prime \prime}\right)$ form a pencil of divisors, and such a pencil has only 2 double points. Thus $\Sigma_{(\Gamma, \theta)}$ cannot be a double conic.

The same argument works for a double line $\tilde{q}^{2} \subset \Sigma_{(\Gamma, \theta)}$ with $q \neq p=t_{1} \cap t_{2}$ : Any point of $\check{q}$ has to be singular, so if we take 3 points $r_{1}, r_{2}, r_{3} \in \check{q}$ we have 3 different intersection points of $t_{1}$ or $t_{2}$ with $\check{r}_{1}, \check{r}_{2}$ and $\check{r}_{3}$. We would need 3 elements in $\mathbb{P}\left(\Gamma_{1}^{\prime \prime}\right)$ or $\mathbb{P}\left(\Gamma_{2}^{\prime \prime}\right)$ being a double point, which is impossible.

So the only possibility left is $\check{p}^{2} \subset \Sigma_{(\Gamma, \theta)}$. In this case for all lines $l$ through $p$ there is a section $s \in \Gamma$ such that there is a $\tilde{s} \in H^{0}(\theta(-2))$ with $s=l^{2} \tilde{s}$.

- $\theta=\mathcal{J}_{1}^{j}(3)$ :

We can assume that $p=(0: 0: 1), t_{1}=\{x=0\}, t_{2}=\{y=0\}$, furthermore $j=2$ and $\eta=(1: 0: 0)$. A section of $\mathcal{J}_{1}^{2}(1)$ is of the form $\tilde{s}=c_{1} y+c_{2} z$. Let a line through $p$ be given by $l=\alpha x+\beta y$, then

$$
l^{2} \cdot \tilde{s}=c_{2} \alpha^{2} x^{2} z+c_{1} \beta^{2} y^{3}+c_{2} \beta^{2} y^{2} z .
$$

Thus $\check{l}$ is a singular point of $\Sigma_{(\Gamma, \theta)}$ if there is a section $s \in \Gamma$ of the form $s=a_{0} x^{2} z+a_{1} y^{3}+a_{2} y^{2} z$ such that there are constants $c_{1}, c_{2}$ with

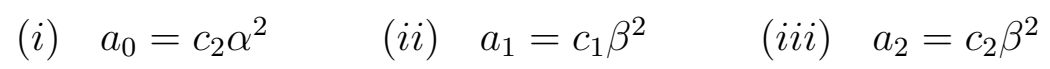

(i) and (iii) give $a_{2}=a_{0} \frac{\beta^{2}}{\alpha^{2}}$. Thus $s$ defines $l$ uniquely. We write $s_{l}$ for a section associated to $l$. Since for $l \neq \tilde{l} \operatorname{span}\left\{s_{l}, s_{\tilde{l}}=\Gamma\right.$, a necessary condition for $\check{p}^{2} \subset \Sigma_{(\Gamma, \theta)}$ is that

$$
\Gamma \subset \operatorname{span}\left\{x^{2} z, y^{3}, y^{2} z\right\}
$$

But $\operatorname{span}\left\{x^{2} z, y^{3}, y^{2} z\right\}$ is a subset of the image of $H^{0}(\theta(-1))$ under the multiplication with $x+y$. Thus by lemma 8.2.5 $p$ is a base point. 
- $\mathcal{O}_{t_{1}}(2) \oplus \mathcal{O}_{t_{2}}(2)$ :

If there is a section $\tilde{s} \in H^{0}(\theta(-2))$ such that $s=l^{2} \tilde{s} \in \Gamma$, the evaluation of $s$ in $\theta_{p}=\mathcal{O}_{p} \oplus \mathcal{O}_{p}$ vanishes. Therefore

$$
e v_{p}: \Gamma \otimes \mathcal{O}_{p} \rightarrow \theta_{p}
$$

is not surjective, which implies that $e v: \Gamma \otimes \mathcal{O} \rightarrow \theta$ is not surjective. Thus $p$ would be a base point.

This completes the proof.

\subsubsection{Lines}

Now we investigate those $\theta$ with $\operatorname{ch}(\theta)=2 h+3 h^{2}$, which are torsion free on their support, such that $\operatorname{set} \operatorname{supp}(\theta)=t$ with $t$ a line in $\mathbb{P}_{2}$. We will describe all these sheaves $\theta$ explicitly and show that for any base point free $(\Gamma, \theta) \in$ $\operatorname{Syst}\left(2 h+3 h^{2}, 2\right), \Sigma_{(\Gamma, \theta)}$ is reduced.

Lemma 8.4.10 Let $\theta$ with $\operatorname{ch}(\theta)=2 h+3 h^{2}$ be torsion free on its support and $\operatorname{setsupp}(\theta)=t$, then there is $i \in \mathbb{Z}$ such that

$$
0 \rightarrow \mathcal{O}_{t}(4-i) \rightarrow \theta \rightarrow \mathcal{O}_{t}(i) \rightarrow 0
$$

\section{Proof:}

If $\operatorname{supp}(\theta)$ is reduced then $\theta$ is a torsion free sheaf on a line and therefore locally free over $\mathcal{O}_{t}$. Since $\operatorname{ch}(\theta) \cdot h=2 h^{2}$ one has $r k(\theta)=2$ considering $\theta$ as an $\mathcal{O}_{t}$-module. Due to a theorem of Grothendieck (see for example [OSS] pp.22) any locally free sheaf on $\mathbb{P}_{1}$ is a direct sum of line bundles, therefore one gets a sequence of the form 8.4.13.

If $\operatorname{supp}(\theta))$ is non reduced, we use the construction of lemma 8.4.4. Consider $\left.\theta\right|_{t}$. It must be $\operatorname{ch}\left(\left.\theta\right|_{l}\right)=h+\left(m+\frac{1}{2}\right) h^{2}$, since $\theta$ is not concentrated on $t$ and torsion free on its support. Therefore $\mathcal{K}$, the kernel of $\left.\theta \rightarrow \theta\right|_{t}$, which musts also be torsion free on its support, is of the form $\mathcal{K}=\mathcal{O}_{t}(2-m)$. Now we consider $\left.T\left(\left.\theta\right|_{t}\right) \hookrightarrow \theta\right|_{t}$ and argue as we did in the proof of lemma 8.4.4.

This completes the proof. 
Lemma 8.4.11 One has

$$
\begin{array}{ccccc}
0 & \rightarrow & \left.\operatorname{Ext}_{\mathcal{O}_{t}}^{1}\left(\mathcal{O}_{t}(4-i), \mathcal{O}_{t}(i)\right)\right) & \rightarrow & \\
& \rightarrow & H^{0}\left(\mathcal{O}_{t}(2 i-3)\right) & \rightarrow &
\end{array}
$$

and the extensions corresponding to $\phi\left(\operatorname{Ext}_{\mathcal{O}_{t}}^{1}\left(\mathcal{O}_{t}(4-i), \mathcal{O}_{t}(i)\right)\right)$ are direct sums of line bundles on $t$.

\section{Proof:}

We apply the Grothendieck spectral sequence of for $\operatorname{Hom}\left(\mathcal{O}_{t}(4-i),-\right)=H^{0}$ 。 $\underline{\operatorname{Hom}}\left(\mathcal{O}_{t}(i),-\right)$ to $\mathcal{O}_{t}(i)$ : One has

$$
\underline{\operatorname{Hom}}_{\mathcal{O}_{t}}\left(\mathcal{O}_{t}(4-i), \mathcal{O}_{t}(i)\right)=\mathcal{O}_{t}(2 i-4)
$$

and a surjective map

$\underline{H o m}_{\mathcal{O}_{\mathbb{P}_{2}}}\left(\mathcal{O}_{t}(4-i), \mathcal{O}_{t}(i)\right) \rightarrow \underline{H o m}_{\mathcal{O}_{\mathbb{P}_{2}}}\left(\mathcal{O}_{t}(4-i), \mathcal{O}_{t}(i)\right) \otimes \mathcal{O}_{t}=\underline{H o m}_{\mathcal{O}_{t}}\left(\mathcal{O}_{t}(4-i), \mathcal{O}_{t}(i)\right)$

Thus we have we have a surjection

$$
\underline{\operatorname{Hom}}_{\mathcal{O}_{\mathbb{P}_{2}}}\left(\mathcal{O}_{t}(4-i), \mathcal{O}_{t}(i)\right) \rightarrow \mathcal{O}_{t}(2 i-4) \rightarrow 0
$$

Applying $\underline{\operatorname{Hom}}\left(\mathcal{O}_{t}(4-i),-\right)$ to the resolution of $\mathcal{O}_{t}(i)$ gives

$$
\begin{aligned}
& 0 \rightarrow \underline{\operatorname{Hom}}_{\mathcal{O}_{\mathbb{P}_{2}}}\left(\mathcal{O}_{t}(4-i), \mathcal{O}_{t}(i)\right) \rightarrow \underline{\operatorname{Ext}}^{1}\left(\mathcal{O}_{t}(4-i), \mathcal{O}(i-1)\right) \rightarrow \\
& \rightarrow \quad \underline{\operatorname{Ext}}^{1}\left(\mathcal{O}_{t}(4-i), \mathcal{O}(i)\right) \rightarrow \underline{\operatorname{Ext}^{1}}\left(\mathcal{O}_{t}(4-i), \mathcal{O}_{t}(i)\right) \rightarrow 0
\end{aligned}
$$

We have $\underline{\operatorname{Ext}}{ }^{1}\left(\mathcal{O}_{t}(4-i), \mathcal{O}(i)\right)=\mathcal{O}_{t}(2 i-3)$ and $\underline{\operatorname{Ext}}{ }^{1}\left(\mathcal{O}_{t}(4-i), \mathcal{O}(i-1)\right)=$ $\mathcal{O}_{t}(2 i-4)$, so we get

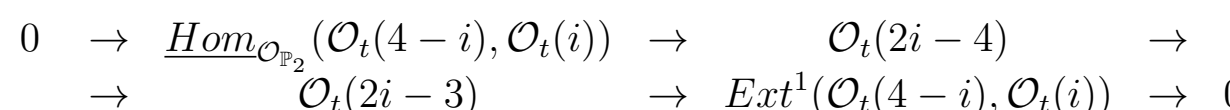

8.4.15 and the first half of 8.4.16 give 


$$
\mathcal{O}_{t}(2 i-4)=\underline{H o m}_{\mathcal{O}_{t}}\left(\mathcal{O}_{t}(4-i), \mathcal{O}_{t}(i)\right) \cong \underline{H o m}_{\mathcal{O}_{\mathbb{P}_{2}}}\left(\mathcal{O}_{t}(4-i), \mathcal{O}_{t}(i)\right)
$$

And therefore the second half of 8.4.16 gives

$$
\underline{\operatorname{Ext}^{1}}\left(\mathcal{O}_{t}(4-i), \mathcal{O}_{t}(i)\right) \cong \mathcal{O}_{t}(2 i-3)
$$

Furthermore

$$
H^{2}\left(\underline{H o m}_{\mathbb{O}_{2}}\left(\mathcal{O}_{t}(4-i), \mathcal{O}_{t}(i)\right)\right) \cong H^{2}\left(\mathcal{O}_{t}(2 i-4)\right)=0
$$

So with 8.4.17, 8.4.18 and 8.4.19 the exact sequence of low degree of the Grothendieck spectral sequence becomes

$$
\begin{aligned}
& 0 \rightarrow H^{1}\left(\mathcal{O}_{t}(2 i-4)\right) \stackrel{\phi}{\rightarrow} \operatorname{Ext}_{\mathcal{O}_{\mathbb{P}_{2}}}^{1}\left(\mathcal{O}_{t}(4-i), \mathcal{O}_{t}(i)\right) \rightarrow \\
& \rightarrow H^{0}\left(\mathcal{O}_{t}(2 i-3)\right) \rightarrow \quad 0
\end{aligned}
$$

Finally we have

$$
H^{1}\left(\mathbb{P}_{2}, \mathcal{O}_{t}(2 i-4)\right) \cong H^{1}\left(t, \mathcal{O}_{t}(2 i-4)\right) \cong \operatorname{Ext}_{\mathcal{O}_{t}}^{1}\left(\mathcal{O}_{t}, \mathcal{O}_{t}(2 i-4)\right)=\operatorname{Ext}_{\mathcal{O}_{t}}^{1}\left(\mathcal{O}_{t}(4-i), \mathcal{O}_{t}(i)\right)
$$

Thus we get 8.4.14. If $i<2$ one has

$$
\left.\operatorname{Ext}_{\mathcal{O}_{t}}^{1}\left(\mathcal{O}_{t}(4-i), \mathcal{O}_{t}(i)\right)\right) \simeq \operatorname{Ext}_{\mathcal{O}_{\mathbb{P}_{2}}}^{1}\left(\mathcal{O}_{t}(4-i), \mathcal{O}_{t}(i)\right)
$$

and for $\left.i \geq 2 \operatorname{Ext}_{\mathcal{O}_{t}}^{1}\left(\mathcal{O}_{t}(4-i), \mathcal{O}_{t}(i)\right)\right)=0$. Any extension of $\mathcal{O}_{t}(4-i)$ by $\mathcal{O}_{t}(i)$ on $t$ is also an extension on $\mathbb{P}_{2}$. Therefore the extensions corresponding to $\phi\left(\operatorname{Ext}_{\mathcal{O}_{t}}^{1}\left(\mathcal{O}_{t}(4-i), \mathcal{O}_{t}(i)\right)\right)$ are direct sums of line bundles on $t$.

This completes the proof.

Now we investigate the case $i \geq 2$, which means $\operatorname{Ext}_{\mathcal{O}_{\mathbb{P}_{2}}}^{1}\left(\mathcal{O}_{t}(4-i), \mathcal{O}_{t}(i)\right) \simeq$ $H^{0}\left(\mathcal{O}_{t}(2 i-3)\right)$. Any element of the Picard group of $\operatorname{Proj}\left(\mathbb{C}[x, y, z] /\left(t^{2}\right)\right)$, which we denote by $2 t$, can be obtained as quotients of line bundles on $\mathbb{P}_{2}$ (see [D]): 


$$
0 \rightarrow \mathcal{O}(i-2) \rightarrow \mathcal{O}(i) \rightarrow \mathcal{O}_{2 t}(i) \rightarrow 0
$$

We can extend this to a diagram

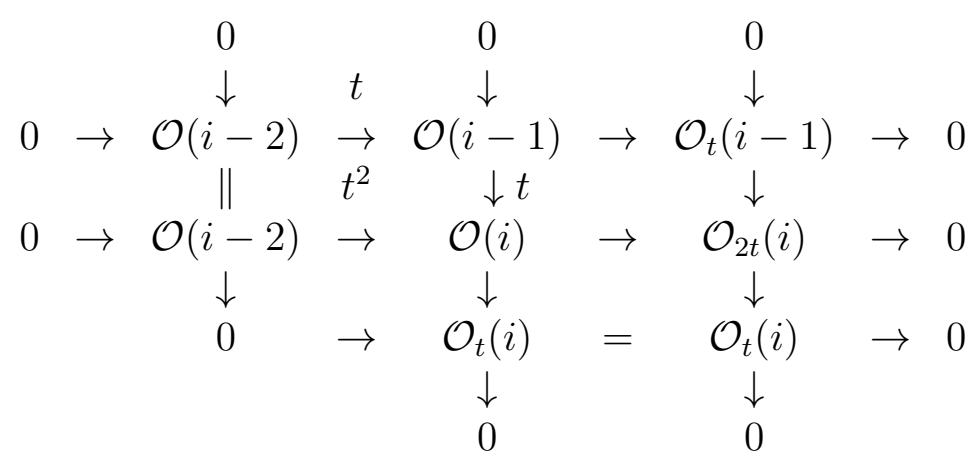

Thus for $\mathcal{O}_{2 t}(i+1)$ we have

$$
0 \rightarrow \mathcal{O}_{t}(i) \rightarrow \mathcal{O}_{2 t}(i+1) \rightarrow \mathcal{O}_{t}(i+1) \rightarrow 0
$$

Lemma 8.4.12 Any $\theta$ that corresponds to an element of $\operatorname{Ext}_{\mathcal{O}_{\mathbb{P}_{2}}}^{1}\left(\mathcal{O}_{t}(4-i), \mathcal{O}_{t}(i)\right)$ for $i \geq 2$ is of the form $\mathcal{I}_{\eta} \otimes \mathcal{O}_{2 t}(i+1)$, where $\mathcal{I}_{\eta}$ is the ideal subsheaf on $\mathcal{O}_{2 t}$ of the Hilbert scheme of points $\eta$ on $t$ with $|\eta|:=\left|\mathcal{O}_{\eta}\right|=2 i-3$.

\section{Proof:}

Let $\eta$ be the zero set of $s_{\eta} \in H^{0}\left(\mathcal{O}_{t}(2 i-3)\right) . s_{\eta}$ induces a map $\mathcal{O}_{t}(4-i) \hookrightarrow$ $\mathcal{O}_{t}(i+1)$. This map extends the sequence 8.4.21 uniquely to a diagram:

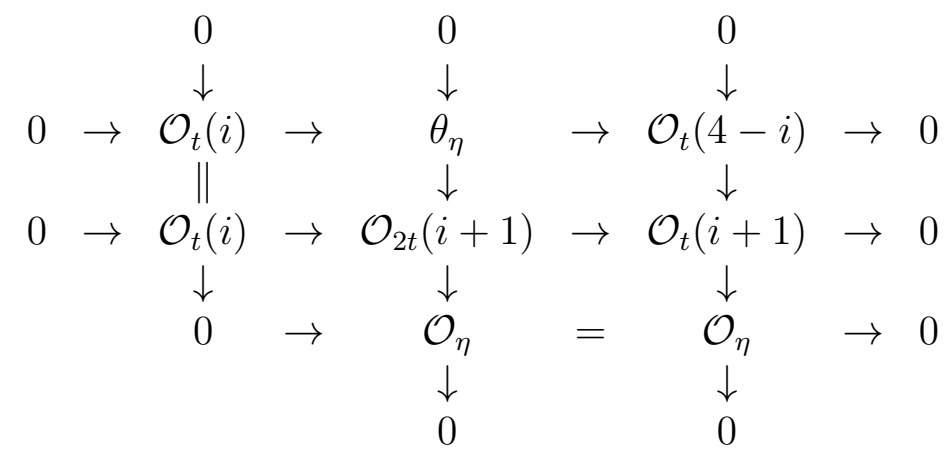

from which we see that $\theta_{\eta}=\mathcal{I}_{\eta}$. For two different Hilbert schemes $\eta, \xi \mathcal{I}_{\eta}$ and $\mathcal{I}_{\xi}$ are not isomorphic: if $\operatorname{setsupp}\left(\mathcal{O}_{\eta}\right) \neq \operatorname{set} \operatorname{supp}\left(\mathcal{O}_{\xi}\right)$ this is obvious, because if restricted to an open set $U$ such that $\operatorname{setsupp}\left(O_{\eta}\right) \cap U=\emptyset$ and $\operatorname{setsupp}\left(O_{\xi}\right) \cap$ 
$U=p$ a point, $\left.\mathcal{I}_{\eta}\right|_{U}$ has one generator while $\left.\mathcal{I}_{\xi}\right|_{U}$ has two. If $\operatorname{setsupp}\left(\mathcal{O}_{\eta}\right)=$ $\operatorname{setsupp}\left(\mathcal{O}_{\xi}\right)$ consider an open set $U$ such that $\left.\mathcal{O}_{\eta}\right|_{U} \neq\left.\mathcal{O}_{\xi}\right|_{U}$, $\operatorname{setsupp}\left(O_{\xi}\right) \cap U=p$ and extend $\left.\mathcal{I}_{\eta}\right|_{U},\left.\mathcal{I}_{\xi}\right|_{U}$ to sheaves $\mathcal{I}_{\tilde{\eta}}, \mathcal{I}_{\tilde{\xi}}$, which are locally free apart from $p$. If $\mathcal{I}_{\eta} \simeq \mathcal{I}_{\xi}$ then $\mathcal{I}_{\tilde{\eta}} \simeq \mathcal{I}_{\tilde{\xi}}$. But since $\mathcal{O}_{\tilde{\eta}} ¥ \mathcal{O}_{\tilde{\xi}}$ one has $\operatorname{ch}\left(\mathcal{I}_{\tilde{\eta}}\right) \neq \operatorname{ch}\left(\mathcal{I}_{\tilde{\xi}}\right)$, which contradicts $\mathcal{I}_{\tilde{\eta}} \simeq \mathcal{I}_{\tilde{\xi}}$.

Since $\operatorname{Ext}_{\mathcal{O}_{\mathbb{P}_{2}}}^{1}\left(\mathcal{O}_{t}(4-i), \mathcal{O}_{t}(i)\right) \simeq H^{0}\left(\mathcal{O}_{t}(2 i-3)\right)$ we obtain with this construction any nontrivial extension of $\mathcal{O}_{t}(4-i)$ by $\mathcal{O}_{t}(i)$.

This completes the proof.

\section{Remarks:}

- Note that $\mathcal{I}_{\eta}$ and $\mathcal{J}_{\eta} \otimes \mathcal{O}_{2 t}$, where $\mathcal{J}_{\eta}$ is the ideal sheaf of $\eta$ on $\mathbb{P}_{2}$, are not isomorphic. Applying $\otimes \mathcal{O}_{2 t}$ to

$$
0 \rightarrow \mathcal{J}_{\eta} \rightarrow \mathcal{O} \rightarrow \mathcal{O}_{\eta} \rightarrow 0
$$

gives

$$
0 \rightarrow \underline{\operatorname{Tor}^{1}}\left(\mathcal{O}_{\eta}, \mathcal{O}_{2 t}\right) \rightarrow \mathcal{J}_{\eta} \otimes \mathcal{O}_{2 t} \rightarrow \mathcal{O}_{2 t} \rightarrow \mathcal{O}_{\eta} \rightarrow 0
$$

Thus with $\underline{\operatorname{Tor}}^{1}\left(\mathcal{O}_{\eta}, \mathcal{O}_{2 t}\right)=\mathcal{O}_{\eta}$ one has

$$
\mathcal{J}_{\eta} \otimes_{\mathcal{O}_{\mathbb{P}_{2}}} \mathcal{O}_{2 t}=\mathcal{I}_{\eta} \oplus \mathcal{O}_{\eta}
$$

which is not torsion free on its support.

- We can identify the global sections of $\mathcal{O}_{2 t}(i)$ with

$$
H^{0}\left(\mathcal{O}_{2 t}(i)\right)=\{p(x, y)+z q(x, y) \mid \operatorname{deg}(p)=i, \operatorname{deg}(q)=i-1\} .
$$

Let the zero set divisor of $s_{\eta}$ be $\sum_{i}\left(\beta_{i}:-\alpha_{i}: 0\right)^{n_{i}}$ then $H^{0}\left(\mathcal{I}_{\eta}(i)\right)$ seen as a subspace of $H^{0}\left(\mathcal{O}_{2 t}(i)\right)$ is

$$
H^{0}\left(\mathcal{I}_{\eta}(i)\right)=\left\{\prod_{i}\left(\alpha_{i} x+\beta_{i} y\right)^{n_{i}} p(x, y)+z q(x, y)|\operatorname{deg}(p)=i-| \eta \mid ; \operatorname{deg}(q)=i-1\right\} .
$$


Lemma 8.4.13 The sheaves $\theta$ with $\operatorname{setsupp}(\theta)=t$ and $\operatorname{supp}(\theta)$ non reduced, that can occur in a semistable coherent system $(\Gamma, \theta) \in \operatorname{Syst}\left(2 h+3 h^{2}, 2\right)$ are of the form $\mathcal{I}_{\eta_{1}}(3)$ and $\mathcal{I}_{\eta_{3}}(4)$, where $\left|\eta_{i}\right|=i$.

\section{Proof:}

Suppopse $\left(\Gamma, \mathcal{I}_{\eta_{2 i+1}}(i+3)\right)$ is semistable. Since we have a sequence

$$
0 \rightarrow\left(\Gamma^{\prime}, \mathcal{O}_{t}(i+2)\right) \rightarrow\left(\Gamma, \mathcal{I}_{\eta_{2 i+1}}(i+3)\right) \rightarrow\left(\Gamma^{\prime \prime}, \mathcal{O}_{t}(2-i)\right) \rightarrow 0
$$

the semistability implies

$$
p_{\left(\Gamma^{\prime}, \mathcal{O}_{t}(i+2)\right)} \leq p_{\left(\Gamma, \mathcal{I}_{\eta_{2 i+1}}(i+3)\right)}
$$

Now recall, that $P_{\mathcal{O}_{t}(j)}(n)=n+j+1$ which one computes from 2.6.4 using $c_{1}\left(\mathcal{O}_{t}(j)\right)=1, c_{2}\left(\mathcal{O}_{t}(j)\right)=1-j$ and therefore $P_{\theta}(n)=2 n+6$. Thus $\Gamma^{\prime} \neq 0$ is only possible if $i=0$. For $i>0$ it must be zero and therefore $\operatorname{dim}\left(\Gamma^{\prime \prime}\right)=2$. But $H^{0}\left(\mathcal{O}_{t}(j)\right)=j+1$, thus we get $i<2$.

So the only sheaves, that can occur in a semistable system $(\Gamma, \theta)$, are $\mathcal{I}_{\eta_{1}}(3)$ and $\mathcal{I}_{\eta_{3}}(4)$. We have to consider all possible inclusions

$$
0 \rightarrow\left(\Gamma^{\prime}, \theta^{\prime}\right) \rightarrow(\Gamma, \theta)
$$

It is impossible to destabilize $(\Gamma, \theta)$, where $\theta$ is either $\mathcal{I}_{\eta_{1}}(3)$ or $\theta=\mathcal{I}_{\eta_{3}}(4)$, with a $\theta^{\prime}$ such that $\operatorname{supp}\left(\theta^{\prime}\right)$ is nonreduced since both sheaves would have the same multiplicity. If $\theta^{\prime}=\mathcal{O}_{t}(j)$ we can choose a $\Gamma$ such that the restriction of no section to $t$ vanishes, so $\Gamma^{\prime}=0$ and therefore $\theta=\mathcal{I}_{\eta_{1}}(3)$ or $\theta=\mathcal{I}_{\eta_{3}}(4)$ occur in a semistable coherent system.

This completes the proof.

Lemma 8.4.14 There are two sheaves $\theta$ with $\operatorname{setsupp}(\theta)=t$ and $\operatorname{supp}(\theta)$ reduced, that can occur in a $(\Gamma, \theta) \in \operatorname{Syst}\left(2 h+3 h^{2}, 2\right)$. These are $\mathcal{O}_{t}(1) \oplus \mathcal{O}_{t}(3)$ and $\mathcal{O}_{t}(2) \oplus \mathcal{O}_{t}(2)$.

\section{Proof:}

Since $\theta$ is torsion free on its support it must be locally free over $\mathcal{O}_{t}$ and is therefore always a direct sum of line bundles. So we have a sequence of the form

$$
0 \rightarrow \mathcal{O}_{C}(4-i) \rightarrow \mathcal{O}_{C}(i) \oplus \mathcal{O}_{C}(4-i) \rightarrow \mathcal{O}_{C}(i) \rightarrow 0
$$


with $i \leq 2$. Considering a coherent system $\left(\Gamma, \mathcal{O}_{C}(i) \oplus \mathcal{O}_{C}(4-i)\right)$ with $i<2$, the sequence of coherent systems induced by 8.4 .22 is

$$
0 \rightarrow\left(\Gamma^{\prime}, \mathcal{O}_{C}(4-i)\right) \rightarrow\left(\Gamma, \mathcal{O}_{C}(i) \oplus \mathcal{O}_{C}(4-i)\right) \rightarrow\left(\Gamma^{\prime \prime}, \mathcal{O}_{C}(i)\right) \rightarrow 0
$$

If $\left(\Gamma, \mathcal{O}_{C}(i) \oplus \mathcal{O}_{C}(4-i)\right)$ is semistable $\operatorname{dim}\left(\Gamma^{\prime \prime}\right)=2$ by the same argument as in the proof of lemma 8.4.13. Thus $i \geq 1$ since we need $H^{0}\left(\mathcal{O}_{C}(i)\right) \geq 2$. So we find $i=1$ or $i=2$ are the only possibilities such that $\left(\Gamma, \mathcal{O}_{C}(i) \oplus \mathcal{O}_{C}(4-i)\right)$ is semistable.

This completes the proof.

Since we now know all possibilities of $\theta$, we can show

Proposition 8.4.15 For any base point free $(\Gamma, \theta) \in \operatorname{Syst}\left(2 h+3 h^{2}, 2\right)$ with $\operatorname{setsupp}(\theta)=t, \Sigma_{(\Gamma, \theta)}$ is reduced.

\section{Proof:}

Once again we use lemma 8.4.1.

- $\theta=\mathcal{O}_{t}(2) \oplus \mathcal{O}_{t}(2)$ or $\theta=\mathcal{O}_{t}(1) \oplus \mathcal{O}_{t}(3)$ :

If the spectral scheme has a non reduced component, then there is a $l \neq t$ such that $\check{l}$ is a singular point. Let $\xi=l \cap t$. So there is a $s=l^{2} \tilde{s} \in \Gamma$ with $\tilde{s} \in H^{0}(\theta(-2))$, but that means $s$ vanishes at $\xi$. Thus $\overline{e v}_{\xi}: \Gamma \rightarrow \theta_{\xi}$ is not surjective, which implies $\overline{e v}$ is not surjective, which would mean that $\xi$ is a base point.

- $\mathcal{I}_{\eta_{1}}(3)$ :

We can choose coordinates such that $\eta_{1}=(0: 1: 0)$, thus

$$
\begin{gathered}
H^{0}\left(\mathcal{I}_{\eta_{1}}(3)\right)=\{x p(x, y)+z q(x, y) \mid \operatorname{deg}(p)=\operatorname{deg}(q)=2\} \\
H^{0}\left(\mathcal{I}_{\eta_{1}}(1)\right)=\left\{c_{1} x+c_{2} z\right\} .
\end{gathered}
$$

Given a line $l=\alpha x+\beta y+\tau z$ and a section $\tilde{s}=c_{1} x+c_{2} z \in H^{0}\left(\mathcal{I}_{\eta_{1}}(1)\right)$, we compute

$l^{2} \cdot \tilde{s}=$ 


$$
\begin{aligned}
= & \left(\alpha^{2} x^{2}+\beta^{2} y^{2}+2 \alpha \beta x y+2 \alpha \tau x z+2 \beta \tau y z\right)\left(c_{1} x+c_{2} z\right)= \\
= & x c_{1}\left(\alpha^{2} x^{2}+\beta^{2} y^{2}+2 \alpha \beta x y\right)+ \\
& +z\left(2 c_{1} \alpha \tau x^{2}+2 c_{1} \beta \tau x y+c_{2}\left(\alpha^{2} x^{2}+\beta^{2} y^{2}+2 \alpha \beta x y\right)\right)= \\
= & x c_{1}(\alpha x+\beta y)^{2}+z\left(\left(2 c_{1} \alpha \tau+c_{2} \alpha^{2}\right) x^{2}+\left(2 c_{1} \beta \tau+2 c_{2} \alpha \beta\right) x y+c_{2} \beta^{2} y^{2}\right)
\end{aligned}
$$

That means, to have a point $\check{l}$, where the spectral scheme is singular on its support, we need a section $s=x p(x, y)+z q(x, y) \in H^{0}\left(\mathcal{I}_{\eta_{1}}(3)\right)$ in $\Gamma$ such that $p$ has a double zero at $\xi=l \cap t$. But since $\Gamma$ is of dimension 2 , there are only two points on $t$, that satisfy this condition. Therefore the only possibilities to have a non reduced component are the lines in $\mathbb{P}_{2}^{*}$ corresponding to the two points $\xi_{1}, \xi_{2} \in t$, for which there is a section $s_{\xi_{j}} \in \Gamma$ with $p_{\xi_{j}}$ having a double zero at $\xi_{j}$.

So $\check{l}$ is a singular point if there is a section

$$
s_{l}=x c_{1}(\alpha x+\beta y)^{2}+z\left(a_{0} x^{2}+a_{1} x y+a_{2} y^{2}\right)
$$

with

(i) $\quad a_{0}=2 c_{1} \alpha \tau+c_{2} \alpha^{2}$

(ii) $\quad a_{1}=2 c_{2} \alpha \beta+2 c_{1} \beta \tau$

(iii) $\quad a_{2}=c_{2} \beta^{2}$.

Now we have to consider two cases:

1. $\beta \neq 0$ :

If for another line $m=\alpha x+\beta y+\tilde{\tau} z \check{m}$ is a singular point, the section $s_{m}$, that ensures this, cannot be an element of $\operatorname{span}\left\{s_{l}\right\}$ : Because if $s_{m}=k s_{l}$ with $k \in \mathbb{C}$, which means, that there are constants $\tilde{c}_{1}, \tilde{c}_{2}$ such that $s_{l}=m^{2}\left(k \tilde{c}_{1} x+k \tilde{c}_{2} z\right)$, then $p_{m}=k p_{l}$ implies $k c_{1}=\tilde{c}_{1}$ and to get the same coefficient at the $y^{2}$ summand of $k q_{l}$ and $q_{m}$ we need $k c_{2}=\tilde{c}_{2}$, thus it is impossible to get the same coefficient at the $x^{2}$ and $x y$ summands of $k q_{l}$ and $q_{m}$, since $\tau \neq \tilde{\tau}$.

Therefore if $\xi=(-\beta: \alpha: 0)$, a necessary condition for $\check{\xi}^{2} \in \Sigma_{(\Sigma, \theta)}$ is, that for all sections $s=x p+z q \in \Gamma$ it is $p(\xi)=0$. But in that case

$$
\overline{e v}_{\xi}: \Gamma \rightarrow \theta_{\xi}
$$

is not surjective and therefore $\xi$ is a base point. 
2. $\beta=0$ :

That means $\eta=\xi$. Now we have

$$
\theta_{\xi}=(x \mathbb{C}[x, z]+z \mathbb{C}[x, z]) / z^{2} \otimes_{\mathbb{C}[x, z]} \mathbb{C}[x, z] /(x, z)=\mathcal{O}_{\xi} \oplus \mathcal{O}_{\xi}
$$

If a section $s \in \Gamma$ is of the form $s=l^{2} \tilde{s}$ the corresponding polynomials $p, q$ have a double zero at $\xi$. Therefore $\overline{e v}_{\xi}\left(s \otimes \mathcal{O}_{\xi}\right)=0$, which means that $\overline{e v}$ is not surjective and $\xi$ would be a base point.

- $\mathcal{I}_{\eta_{3}}(4)$ :

Let $\eta_{3}$ be given by the zeros of the polynomial $r(x, y)$ of degree 3 . Then

$$
\begin{gathered}
H^{0}\left(\mathcal{I}_{\eta_{3}}(4)\right)=\{r(x, y) p(x, y)+z q(x, y) \mid \operatorname{deg}(p)=1 ; \operatorname{deg}(q)=3\} \\
H^{0}\left(\mathcal{I}_{\eta_{3}}(2)\right)=\{z \tilde{q} \mid \operatorname{deg}(\tilde{q})=1\} .
\end{gathered}
$$

Thus to have a singular point in the spectral scheme apart from $\check{t}$ we need a section $s_{1}=z q_{1}(x, y) \in H^{0}\left(\mathcal{J}_{\eta_{3}}(4)\right)$ in $\Gamma$. Taking a second section $s_{2}=$ $r(x, y) p_{2}(x, y)+z q_{2}(x, y) \in \Gamma$ such that $\Gamma=\operatorname{span}\left\{s_{1}, s_{2}\right\}$ every section $s \in \Gamma$ is of the form

$$
s=r(x, y) p_{2}(x, y)+z q(x, y)
$$

Thus denoting the Hilbert scheme given by the zeros of $r(x, y) p_{2}(x, y)$ by $\eta_{4}$ we see that

$$
\overline{e v}(\Gamma \otimes \mathcal{O}) \subset \mathcal{I}_{\eta_{4}}(4) \subsetneq \mathcal{I}_{\eta_{3}}(4)
$$

which by lemma 8.2.5 means, that there would be a base point.

This completes the proof.

Finally using the lemma of Artamkin, the circumstance that there are instantons in $\tilde{\mathcal{M}}_{n c}^{\text {reg }}(4)$ such that $[A, B]$ is not nilpotent, nilpotent with $[A, B]^{2} \neq 0$, nilpotent with $[A, B]^{2}=0$ and Propositions 8.4.3, 8.4.9 and 8.4.15 gives

Theorem 8.4.16 There are $3 \mathfrak{G}$-orbits in $\tilde{\mathcal{M}}_{n c}^{r e g}(4)$. 


\section{Chapter 9}

\section{Further investigations}

Even though we did not find non reduced spectral schemes for elements of $\tilde{\mathcal{M}}_{n c}^{r e g}(n)$ for $n \leq 4$, the suggestion, that this might be true for any $n$, is false. In [Sch2] Schwarzenberger gave an example of a $\mu$-stable locally free sheaf $E$ on $\mathbb{P}_{2}$ with $\left(c_{1}, c_{2}\right)=(0,6)$, that has a conic as spectral curve. Remarkable in that case is that even though the spectral curve is a conic and $c_{2}(E)=6$, the restriction of $E$ to a jumping line $l$ is $\left.E\right|_{l}=\mathcal{O}_{l}(2) \oplus \mathcal{O}_{l}(-2)$.

For the investigation of charge 5 the construction of coherent systems fails, since one has $\chi(\mathcal{F}(1))=1$ and it does not seem to be the case that $h^{1}(E) \geq 1$ for any locally free $E \in \tilde{\mathfrak{M}}(5)$. A first approach could be to investigate, whether the existence of a line as a component of the spectral curve of $\mathcal{F} \in \tilde{\mathfrak{M}}(5)$ implies, that $\mathcal{F}$ is not locally free. The inverse implication is true, because the dual of $\left.\operatorname{setsupp}\left(\underline{\operatorname{Ext}}{ }^{1}(\mathcal{F}, \mathcal{O})\right)\right)$ belongs to the spectral curve. If this turns out to be true one also gets for charge 5 the non existence of spectral schemes with non reduced components, since the resulting spectral curve of any such scheme has a line as component.

The identification of instantons with coherent systems could lead to a successful investigation of charge 8 , since in that case one has $\chi(\mathcal{F}(2))=2$. But their treatment will be more difficult since the coherent systems are supported on curves of degree 4 . 


\section{References}

[A1] Artamkin, I.V.: Action of biregular automorphism of the affine plane on pairs of matrices, Izvestija Math., 33, No. 2, pp. 433-439, 1989.

[A2] Artamkin, I.V.: Stable bundles on minimal rational surfaces and the Cremona group, Doklady Mathematics, Vol 66, No. 1, pp. 66-71, 2002.

[B1] Barth, W.: Moduli of vector bundles on the projective plane, Inventiones math., 42, pp. 63-91, 1977.

[B2] Barth, W.: Some properties of stable rank-2 vector bundles on $\mathbb{P}_{n}$, Math Ann., 226, pp. 125-150, 1977.

[Bu] Buchdahl, N.: Monads and bundles on rational surfaces, Rocky Mountain Journal, 42, No. 2, 2004.

[DK] Donaldson, S.K., Kronheimer, P.B.: The geometry of four-manifolds, Oxford Math. Monographs, Oxford Univ. Press, 1990.

[GH] Griffiths, P., Harris, J.: Principles of Algebraic Geometry, Wiley, New York, 1978.

[H] Hartshorne, R.: Algebraic geometry, Graduate Texts in Math., SpringerVerlag, Nwe York, 1977.

[K] Kobayashi, S.: Differential geometry of complex vector bundles, Princeton Univ. Press, 1987.

[LP1] LePotier, J.: Systemes coherents et structures de niveau, Asterisque, 214, 1993.

[LP2] LePotier, J., Tikhomirov, A.: sur le morphisme de Barth, arXiv:math0003016vl, 2000.

[LP3] LePotier, J.: Lectures on vector bundles, Cambridge studies in advanced math. 54, Cambridge Univ. Press, 1997.

[LPH] Hulek, M., Lepotier, J.: Sur l'espace de modules des faisceaux stables de rang 2 , de classes Chern (0,3) sur $\mathbb{P}_{2}$, Annales de l'Institut Fourier, 39, pp. 251-292, 1989.

[MFK] Mumford, D., Fogarty, J., Kirwan, F.: Geometric invariant theory, Ergebnisse der Mathematik und ihrer Grenzgebiete 34, Springer-Verlag, 1994. 
[N] Nakajima, H: Lectures on Hilbert schemes of points on surfaces, American Mathematical Society, 1999.

[OSS] Okonek, Schneider, Spindler: Vector bundles on complex projective spaces, Progress in Math. 3, Birkhäuser, 1980.

[Sch1] Schwarzenberger, R.L.E.: Vector bundles on the projective plane 1, Proc. London math. soc., 11, pp. 601-622, 1961.

[Sch2] Schwarzenberger, R.L.E.: Vector bundles on the projective plane 2, Proc. London math. soc., 11, pp. 623-640, 1961.

[Sh1] Shafarevich, I.R.: On some infinite-dimensional groups, Rend. Math. e Appl., 25, pp. 208-212, 1966.

[Sh2] Shafarevich, I.R.: Basic algebraic geometry, Die Grundlehren der math. Wissenschaften in Einzeldarstellungen 213, Springer-Verlag, 1974. 


\section{Lebenslauf}

\section{Persönliche Daten}

Michael Miesener

Mittelweg 5

37133 Friedland

Geboren am 22.11.1978 in Rinteln

Verheiratet, deutsch

\section{Studium}

seit $10 / 2009$

Wissenschaftlicher Mitarbeiter am Mathematischen Institut der Georg-August-Universität

04/2008 - 09/2009 Wissenschaftlicher Mitarbeiter am Mathematischen Institut der Georg-August-Universität im E-Learning Project

12/2007 - 03/2008 Wissenschaftliche Hilfskraft am Mathematischen Institut der Georg-August-Universität im E-Learning Project

04/2005 - 09/2007 Wissenschaftlische Hilfskraft am Mathematischen Institut der Georg-August-Universität

12/2004 - 11/2007 Stipendiat im Graduiertenkolleg "Gruppen und Geometrie" an der Georg-August-Universität

seit $12 / 2004$

Promotionssstudium bei Prof. Dr. Pidstrygach am Mathematischen Institut der Georg-August Universität

$05 / 2004$

Diplom Mathematik an der Ludwig-Maximilian-Universität München

10/2001 Vordiplom Mathematik an der Ludwig-MaximilianUniversität München

04/2001 Vordiplom Physik an der Ludwig-MaximilianUniversität München

\section{Schulbildung}

$1998 \quad$ Abitur

1991 - 1998 Gymnasium Ernestinum in Rinteln

1989 - 1991 Orientierungsstufe in Rinteln

1985 - 1989 Grundschule in Rinteln 